\title{
Quantitative linearization results for the Monge-Ampère equation
}

\author{
Michael Goldman* Martin Huesmann $^{\dagger} \quad$ Felix Otto ${ }^{\ddagger}$
}

April 30, 2021

\begin{abstract}
This paper is about quantitative linearization results for the Monge-Ampère equation with rough data. We develop a large-scale regularity theory and prove that if a measure $\mu$ is close to the Lebesgue measure in Wasserstein distance at all scales, then the displacement of the macroscopic optimal coupling is quantitatively close at all scales to the gradient of the solution of the corresponding Poisson equation. The main ingredient we use is a harmonic approximation result for the optimal transport plan between arbitrary measures. This is used in a Campanato iteration which transfers the information through the scales.
\end{abstract}

\section{Contents}

1 Introduction 2

1.1 Strategy of the proofs $\ldots \ldots \ldots \ldots 7$

1.1.1 The harmonic approximation theorem . . . . . . . . . 7

1.1 .2 Proof of Theorem $1.2 \ldots \ldots \ldots \ldots$

1.2 Comparison with the first part of $[25] \ldots \ldots \ldots \ldots$

$\begin{array}{lll}2 & \text { Preliminaries } & 15\end{array}$

2.1 Notation . . . . . . . . . . . . . . . . . . . . . . . . . . . 15

2.2 Elliptic estimates . . . . . . . . . . . . . . . . . . 16

2.3 The optimal transport problem . . . . . . . . . . . . . . . . . . . 21

2.3 .1 A few technical results . . . . . . . . . . . . . . . . . . 24

$2.3 .2 \quad$ An $L^{\infty}$ bound on the displacement . . . . . . . . . . . . . . 25

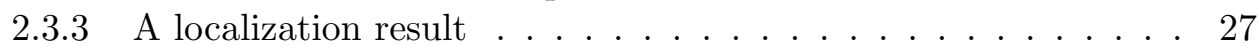

*Université de Paris, CNRS, Sorbonne-Université, Laboratoire Jacques-Louis Lions (LJLL), F-75005 Paris, France, michael.goldman@u-paris.fr

${ }^{\dagger}$ Martin Huesmann, Universität Münster, Einsteinstraße 62, 48149 Münster, Germany, martin.huesmann@uni-muenster.de

${ }^{\ddagger}$ Max Planck Institute for Mathematics in the Sciences, 04103 Leipzig, Germany, Felix.Otto@mis.mpg.de 
\begin{tabular}{|lll}
\hline 3 & The harmonic approximation result & 31
\end{tabular}

3.1 Choice of a good radius . . . . . . . . . . . . . . . . 31

3.1 .1 Unregularized fluxes $\ldots \ldots \ldots$. . . . . . . . . . . 31

3.1.2 Regularized fluxes and good radius for the approximate orthog-

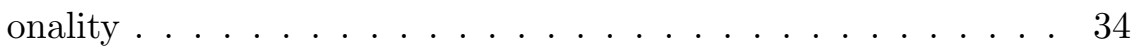

3.1 .3 Good radius for the construction . . . . . . . . . . . . . . . . 37

3.1.4 Choice of the good radius and definition of $\Phi$ and $\phi$. . . . . . 41

3.2 Eulerian version of the harmonic approximation result . . . . . . . . . 43

$3.2 .1 \quad$ Approximate orthogonality . . . . . . . . . . . . . . 44

3.2 .2 The construction . . . . . . . . . . . . . . . . . . . . . 48

3.3 Proof of the harmonic approximation result $\ldots \ldots \ldots$. . . . . . . . 53

\begin{tabular}{|ll|l}
4 & Quantitative bounds on the displacement & 54
\end{tabular}

4.1 The Campanato iteration . . . . . . . . . . . . . . . . . 55

4.2 Choosing good radii $\ldots \ldots \ldots \ldots \ldots$. . . . . . . . . . . . . . 57

4.3 Flux of solutions to Poisson equations on concentric annuli . . . . . . 60

4.4 Linearization on nearby scales . . . . . . . . . . . . . . . . . 62

4.5 Representation of the cumulative shift . . . . . . . . . . . . 66

4.6 Proof of Theorem $\mid 1.2 \ldots \ldots \ldots \ldots$. . . . . . . . . . . . . . 68

\section{Introduction}

Motivated by the optimal matching problem (see for instance [33, 11, 6, 29]), we develop a large-scale regularity theory for optimal couplings between an arbitrary measure and the Lebesgue measure. We start by giving a simplified version of our main result which already gives a good idea of what we achieved. For this we fix a radial cutoff function

$$
\eta \in C_{c}^{\infty}\left(B_{1}\right) \text { with } \int_{\mathbb{R}^{d}} \eta=1, \quad \text { and set } \eta_{R}:=\frac{1}{R^{d}} \eta\left(\frac{\cdot}{R}\right) .
$$

We will work under the assumption that $\sup \left|\nabla^{2} \eta\right| \leq C$ for some fixed universal constant $C>0$ depending only on the dimension. We introduce the following notation: for $R>0$ and $\mu$ a positive measure on $\mathbb{R}^{d}, W_{B_{R}}^{2}(\mu, \kappa):=W^{2}\left(\mu\left\llcorner B_{R}, \kappa d x\left\llcorner B_{R}\right)\right.\right.$ where $W$ is the 2 -Wasserstein distance (see 2.17) for a definition) and $\kappa=\frac{\mu\left(B_{R}\right)}{\left|B_{R}\right|}$ is the generic constant for which this makes sense.

Corollary 1.1. For every $0 \leq \alpha<1$, there is a constant $C>0$ depending only on the dimension $d$ and $\alpha$ such that the following holds: consider $\bar{R} \geq C$ and a positive measure $\mu$ on $B_{\bar{R}}$ with $\mu\left(B_{\bar{R}}\right)=\left|B_{\bar{R}}\right|$ and such that

$$
\frac{1}{\left|B_{R}\right|} W_{B_{R}}^{2}(\mu, \kappa) \leq R^{\alpha} \quad \forall R \in[1, \bar{R}]
$$

Let $u$ be a (distributional) solution of the Poisson equation (here $\nu$ denotes the exterior normal to $\left.\partial B_{\bar{R}}\right)$

$$
\Delta u=\mu-1 \quad \text { in } B_{\bar{R}} \quad \text { and } \quad \nu \cdot \nabla u=0 \quad \text { on } \partial B_{\bar{R}} .
$$


Then, if $\pi$ is the optimal coupling for $W_{B_{\bar{R}}}(\mu, 1)$, for $\bar{R} \geq R \geq C$,

$$
\left|\int_{\mathbb{R}^{d} \times \mathbb{R}^{d}} \eta_{R}(x)\left(y-x-\int_{\mathbb{R}^{d}} \eta_{R} \nabla u\right) d \pi\right| \leq \frac{C}{R^{1-\alpha}} .
$$

This result gives a precise and quantitative description of the well-known fact that the Monge-Ampère equation linearizes to the Poisson equation around the Lebesgue measure (see for instance [6, 14]). Indeed, (1.3) shows that provided the measure $\mu$ is close to the Lebesgue measure at every scale in the sense of $(1.2)$, then the displacement $y-x$ is close to $\nabla u$ on average, that is, in a weak topology.

Let us comment on the scaling in (1.3). As discussed above, (1.3) amounts to the estimate of a linearization error. Since the left-hand side obviously is linear in the displacement, one would generically expect the right-hand side to be quadratic in the displacement. Obviously, $\frac{1}{\left|B_{R}\right|} W_{B_{R}}^{2}(\mu, \kappa)$ is also a quadratic expression in the displacement. However, in terms of dimensions, the left-hand side of (1.3) has units of length, so also the right-hand side has to have units of length. This leaves only the expression $\frac{1}{R\left|B_{R}\right|} W_{B_{R}}^{2}(\mu, \kappa)$, which is consistent with 1.2 . Setting $\beta(R):=R^{\alpha}$, another way to read $(1.3)$ is to divide it by the displacement scale $\sqrt{\beta(R)}$, so that the left-hand side turns into the relative linearization error and the right-hand side assumes the non-dimensional form $\frac{\sqrt{\beta(R)}}{R} \ll 1$. However, in proving this, it is crucial that the dimensional quantity $\frac{\beta(R)}{R}$ is decreasing with increasing $R$, to the point that the sum over $\frac{\beta\left(R_{k}\right)}{R_{k}}$ for geometrically decaying $\left(R_{k}\right)_{1 \leq k \leq K}$ is dominated by the smallest scale, see (1.7) below. This explains why we require $\beta(R) \ll R$ (i.e. $\alpha<1$ ) rather than the dimensionally more natural $\beta(R) \ll R^{2}$.

Let us now introduce some notation and state our first main result. Let $\pi$ be a measure on $\mathbb{R}^{d} \times \mathbb{R}^{d}$ with cyclically monotone support. For a given $\bar{R}>0$, let $\mu:=\pi_{1}\left\llcorner B_{\bar{R}}\right.$ and assume that $\pi_{2}\left\llcorner B_{\bar{R}}=d x\right.$, where we denoted by $\pi_{i}$ the marginals of $\pi$. For $t \in[0,1], \zeta \in C_{c}^{0}\left(\mathbb{R}^{d}\right)$, and $\xi \in C_{c}^{0}\left(\mathbb{R}^{d}\right)^{d}$, define the flux-density pair

$$
\begin{aligned}
\int_{\mathbb{R}^{d}} \zeta d \rho_{t}=\int_{\mathbb{R}^{d} \times \mathbb{R}^{d}} \zeta((1-t) x+t y) d \pi & \\
& \text { and } \int_{\mathbb{R}^{d}} \xi \cdot d j_{t}=\int_{\mathbb{R}^{d} \times \mathbb{R}^{d}} \xi((1-t) x+t y) \cdot(y-x) d \pi .
\end{aligned}
$$

We then let

$$
\bar{j}:=\int_{0}^{1} d j(\cdot, t)
$$

be the time integral (see 2.1) ) of the flux $j$. Notice that $(\rho, j)$ solves in a distributional sense the continuity equation

$$
\partial_{t} \rho+\nabla \cdot j=0 \quad \text { and } \quad \rho_{0}=\mu, \rho_{1}=1 .
$$

We will fix for the whole paper a nonnegative rate function $\beta(R)$ satisfying the following hypothesis: it is increasing, $R \mapsto \frac{\beta(R)}{R}$ is decreasing, and there exists a 
constant $C_{\beta}>0$ such that

$$
\sum_{\ell \geq 0} \frac{\beta\left(2^{\ell} R\right)}{2^{\ell} R} \leq C_{\beta} \frac{\beta(R)}{R} \quad \text { for every } R \geq 1 \quad \text { and } \quad \beta(1) \leq C_{\beta} .
$$

We do not claim optimality of these conditions. They are rather made to include both the choices $\beta(R)=R^{\alpha}$ for $0 \leq \alpha<1$ and $\beta(R)=\log (R+C)$ (for ${ }^{17} R \gg 1$ ).

Theorem 1.2. Let $\mu$ be a positive measure on $\mathbb{R}^{d}$ such that for some $\bar{R} \gg 1$,

$$
\frac{1}{\left|B_{\bar{R}}\right|} \int_{\left(B_{\bar{R}} \times \mathbb{R}^{d}\right) \cup\left(\mathbb{R}^{d} \times B_{\bar{R}}\right)}|x-y|^{2} d \pi \leq \beta(\bar{R})
$$

and

$$
\frac{1}{\left|B_{R}\right|} W_{B_{R}}^{2}(\mu, \kappa) \leq \beta(R) \quad \forall R \in[1, \bar{R}] .
$$

Then there exists $\bar{R}^{\prime} \sim \bar{R}$ such that letting $u$ be a (distributional) solution of the Poisson equation (recall the definition (1.5) of $\bar{j}$ )

$$
\Delta u=\mu-1 \quad \text { in } B_{\bar{R}^{\prime}} \quad \text { and } \quad \nu \cdot \nabla u=\nu \cdot \bar{j} \quad \text { on } \partial B_{\bar{R}^{\prime}}
$$

and for $R>0$ (recall the definition (1.1) of $\eta_{R}$ ),

$$
h_{R}:=\int_{\mathbb{R}^{d}} \eta_{R} \nabla u
$$

there holds for $\bar{R} \gtrsim R \gtrsim 1$,

$$
\begin{aligned}
\left|\int_{\mathbb{R}^{d} \times \mathbb{R}^{d}} \eta_{R}(x)\left(y-x-h_{R}\right) d \pi\right|+\left|\int_{\mathbb{R}^{d} \times \mathbb{R}^{d}} \eta_{R}\left(y-h_{R}\right)\left(y-x-h_{R}\right) d \pi\right| & \\
& \lesssim \frac{\beta(R)}{R},
\end{aligned}
$$

and

$$
\begin{aligned}
\sup \left\{\left|y-x-h_{R}\right|:(x, y) \in \operatorname{Spt} \pi \cap\left(( B _ { R } \times \mathbb { R } ^ { d } ) \cup \left(\mathbb{R}^{d}\right.\right.\right. & \left.\left.\left.\times B_{R}\left(h_{R}\right)\right)\right)\right\} \\
& \lesssim R\left(\frac{\beta(R)}{R^{2}}\right)^{\frac{1}{d+2}} .
\end{aligned}
$$

For our future work on the optimal matching problem, the following observations will be useful:

Remark 1.3. (i) It will be clear from the proof of Theorem 1.2 that we do not need the hypothesis (1.9) to hold for every radius $R \in[1, \bar{R}]$ but that it is enough to have it for a sequence of approximately geometrically decaying radii i.e. for a sequence $R_{0}=\bar{R} \geq \cdots \geq R_{K} \gg 1$ with $R_{k-1} \geq 2 R_{k} \gtrsim R_{k-1}$;

${ }^{i}$ The notation $A \ll 1$, which we only use in assumptions, means that there exists an $\varepsilon>0$ only depending on the dimension and on $C_{\beta}$, such that if $A \leq \varepsilon$ then the conclusion holds. Similarly, the notation $A \lesssim B$, which we use in output statements, means that there exists a global constant $C>0$ depending on the dimension and on $C_{\beta}$ such that $A \leq C B$. 
(ii) in the statement of Theorem 1.2, we have set the 'small' scale to be equal to one. By scaling, we see that for every $\underline{R}>0$, letting $\underline{\beta}(R):=\underline{R}^{2} \beta(R / \underline{R})$, if hypothesis (1.8)\&(1.9) hold with $\beta$ instead of $\beta$ and for $R \in[\underline{R}, \bar{R}]$ for some $\bar{R} \gg \underline{R}$, then the conclusions $(1.11) \&(1.12)$ also hold for $\bar{R} \gtrsim R \gtrsim \underline{R}$ (still with $\underline{\beta}$ instead of $\beta$ ).

As Corollary 1.1 does, Theorem 1.2 states that if the averaged square displacement is small in the sense of (1.8) at the macroscopic scale $\bar{R}$ and if the measure $\mu$ is close to the Lebesgue measure in the sense of (1.9) at every scale from the macroscopic one $\bar{R}$ to the microscopic one which is set to unity, then the displacement is quantitatively close to $\nabla u$ both in a weak sense (see (1.11) ) and in a strong sense (see (1.12).

As mentioned above, our motivation for proving Theorem 1.2 comes from the optimal matching problem. When $\mu$ is given by a Poisson point process, it is known since [1] (see also [6] and [25]) that for $d=2, \sqrt{1.9}$ holds with $\beta(R)=\log (R+C$ ) with very high probability while for $d \geq 3,11.9$ holds for $\beta(R)=C$ also with very high probability. As will be shown in a future work, (1.11) gives a rigorous validation, at the level of the displacement and down to the microscopic scale, of the ansatz used by Caracciolo et al. in [17]. From this point of view, (1.11) should be seen as a quenched result separating the deterministic from the stochastic part of the analysis. We refer to [6] and the recent paper [5] for a justification of this ansatz at the macroscopic scale on the level of the transport cost respectively the transport map (and to [28, 29] for application of these ideas in the case of Gaussian matching).

In a future work we will also prove that for $d=2,\left|h_{R}\right|^{2} \sim \log \bar{R}$ for $R \sim 1$ (notice that $\log \bar{R} \gg 1$ ) with very high probability and thus $(1.12)$ gives the expected deviation for the transport from the identity plus a shift. In higher dimension, since $\left|h_{R}\right| \sim 1$ for $R \sim 1$ this estimate is less meaningful (see [27]). In another future work, we would also like to address the thermodynamic limit in the matching problem; this will require controlling how the optimal coupling depends on changing the positions of (distant) points. This relates to the question of how sensitively, in terms of a Hölder modulus of continuity, the coupling depends on the measure (see [5, 12, 30]). In our setting, we hope that our large-scale regularity theory might lead to more precise statements.

Before explaining the strategy/main ideas for the proof of Theorem 1.2 we would like to single out one ingredient which can be seen as our second main result. It is a quantitative harmonic approximation result for the displacement $y-x$ under the optimal coupling between two arbitrary measures.

Let $\mu$ and $\lambda$ be two positive measures on $\mathbb{R}^{d}$ and let $\pi$ be a coupling between $\mu$ and $\lambda$ with monotone support. For a given scale $R$, we introduce the local energy

$$
E(R):=\frac{1}{\left|B_{6 R}\right|} \int_{\left(B_{6 R} \times \mathbb{R}^{d}\right) \cup\left(\mathbb{R}^{d} \times B_{6 R}\right)}|x-y|^{2} d \pi
$$

and the local measure of the squared distance between the data and the Lebesgue measure

$$
D(R):=\frac{1}{\left|B_{6 R}\right|} W_{B_{6 R}}^{2}\left(\mu, \kappa_{\mu}\right)+\frac{R^{2}}{\kappa_{\mu}}\left(\kappa_{\mu}-1\right)^{2}+\frac{1}{\left|B_{6 R}\right|} W_{B_{6 R}}^{2}\left(\lambda, \kappa_{\lambda}\right)+\frac{R^{2}}{\kappa_{\lambda}}\left(\kappa_{\lambda}-1\right)^{2} .
$$


The harmonic approximation theorem states that provided these two quantities are small (at the natural scale, i.e. in a dimensionless sense) for some radius $\bar{R}>0$, then there exists a harmonic gradient field $\nabla \Phi$ such that the displacement $y-x$ is very close to $\nabla \Phi$ in $B_{\bar{R}}$.

Theorem 1.4. For every $0<\tau \ll 1$, there exist $\varepsilon(\tau)>0$ and $C(\tau)>0$ such that provided $\frac{1}{\bar{R}^{2}} E(\bar{R})+\frac{1}{\bar{R}^{2}} D(\bar{R}) \leq \varepsilon$ the following holds. There exists a radius $R \in(3 \bar{R}, 4 \bar{R})$ such that if $\Phi$ is the up to an additive constant unique (distributional) solution of

$$
\Delta \Phi=c \text { in } B_{R} \quad \text { and } \quad \nu \cdot \nabla \Phi=\nu \cdot \bar{j} \text { on } \partial B_{R},
$$

where $\bar{j}$ is defined in (1.5) and $c$ is the generic constant for which this equation is solvable, then

$$
\frac{1}{\left|B_{\bar{R}}\right|} \int_{\left(B_{\bar{R}} \times \mathbb{R}^{d}\right) \cup\left(\mathbb{R}^{d} \times B_{\bar{R}}\right)}|x-y+\nabla \Phi(x)|^{2} d \pi \leq \tau E(\bar{R})+C D(\bar{R})
$$

and

$$
\bar{R}^{2} \sup _{B_{2} \bar{R}}\left|\nabla^{2} \Phi\right|^{2}+\sup _{B_{2} \bar{R}}|\nabla \Phi|^{2} \lesssim E(\bar{R})+D(\bar{R}) .
$$

Remark 1.5. If one of the two measures, say the target measure $\lambda$, is the Lebesgue measure, then the same conclusion holds replacing the definition (1.14) of $D(R)$ by $D^{\prime}(R):=\frac{1}{\left|B_{6 R}\right|} W_{B_{6 R}}^{2}(\mu, \kappa)$, see Remark 2.11 for more details.

Most of the paper is dedicated to the proof of this far-reaching generalization of [26. Prop. 4.7]. Let us stress that since $E(\bar{R})$ behaves like a squared $H^{1}$ norm in terms of the Kantorovich potentials and since the squared Wasserstein distance $D(\bar{R})$ behaves like a squared $H^{-1}$ norm (cf. [35, Sec. 7.6]), all quantities occur in the estimate (1.16) as if we were dealing with a second order linear elliptic equation and looking at squared $L^{2}$-based quantities. Anticipating a bit, we point out that having as boundary flux $\nu \cdot \bar{j}$ on $\partial B_{R}$ for $\nabla \Phi$ will play a crucial role in the derivation of Theorem 1.2 from Theorem 1.4. For other applications, such as the small-scale regularity theory developped in [26], it only matters that $\nabla \Phi$ is harmonic and that (1.16) and (1.17) hold (in fact we need also an estimate on $\sup _{B_{\bar{R}}}\left|\nabla^{3} \Phi\right|$ but this follows from harmonicity).

We would also like to comment on the fact that the right-hand side of $(1.15)$ is a constant and not $\mu-\lambda$ as could be expected from (1.10) (see also [26]). This is due to the fact that we need (interior) $L^{2}$ bounds on $\nabla \Phi$ which are generally not true for solutions of the Poisson equation with rough right-hand side. Similar issues were tackled in [6, 28] by mollification with smooth kernels (the heat and the Mehler kernel respectively).

Finally, we should say that while [26, Prop. 4.7] served the purpose of an alternative proof for the partial regularity result of [22] (see also [20]), it is not clear to us that the approach therein based on Caffarelli's regularity theory (see [16, 15]) could also be used in the present context. 


\subsection{Strategy of the proofs}

In this section, we will explain the key ideas for the proofs of our two main theorems. The rough picture is as follows: The first step in the proof of Theorem 1.2 is the harmonic approximation result Theorem 1.4 itself. As in [26], this allows to run a Campanato-style iteration scheme which transfers the information (1.8), namely that the displacement is controlled at the macroscopic scale (here $\bar{R}$ ) down to the microscopic scale (here 1), provided the data are well-behaved in the sense of $(1.9)$. Due to the iterative application of Theorem 1.4 this results into a cumulative shift. The last ingredient for the proof of Theorem 1.2 is the fact that to leading order, this shift is equal to the flux averaged over a microscopic region of the solution of the linearized equation i.e. the Poisson equation, at the macroscopic scale.

This is inspired by the approach of Avellaneda and Lin [10] to a regularity theory for (linear) elliptic equations with periodic coefficients: The good regularity theory of the homogenized operator, i.e. the regularity theory on the thermodynamic scale, is passed down to the scale of the periodicity. This approach has been converted from a qualitative to a quantitative one in the seminal work of Armstrong and Smart [9], in the context of stochastic homogenization. It has been further refined in this context, for instance by Gloria, Neukamm and the last author [24] (see also 8]).

The Campanato iteration driving the approach of Avellaneda and Lin was originally devised for comparing a nonlinear variational problem to its linearization across scales, like the minimal surface problem. Thus not surprisingly, large-scale regularity has been applied also to nonlinear problems in homogenization, see [7, 23] for recent work.

Let us now describe the different steps for the proofs of Theorems 1.4 and 1.2 in more detail. We start with the harmonic approximation result.

\subsubsection{The harmonic approximation theorem}

Since all the quantities appearing in Theorem 1.4 have the same scaling, it is enough to prove it for $\bar{R}=1$. We use here the short-hand notation

$$
E:=E(1)=\frac{1}{\left|B_{6}\right|} \int_{\left(B_{6} \times \mathbb{R}^{d}\right) \cup\left(\mathbb{R}^{d} \times B_{6}\right)}|x-y|^{2} d \pi
$$

and

$$
D:=D(1)=\frac{1}{\left|B_{6}\right|} W_{B_{6}}^{2}\left(\mu, \kappa_{\mu}\right)+\frac{1}{\kappa_{\mu}}\left(\kappa_{\mu}-1\right)^{2}+\frac{1}{\left|B_{6}\right|} W_{B_{6}}^{2}\left(\lambda, \kappa_{\lambda}\right)+\frac{1}{\kappa_{\lambda}}\left(\kappa_{\lambda}-1\right)^{2} .
$$

As in [26], the proof is done at the Eulerian (or Benamou-Brenier) level. Notice that this is somewhat reminiscent of the regularity theory for the pressure field in incompressible Euler equations [2, 13, 32]. Recalling the definition (1.4) of the density-flux pair $(\rho, j)$, we will show the following

Proposition 1.6. For every $0<\tau \ll 1$, there exist positive constants $\varepsilon(\tau)>0$ and $C(\tau)>0$ such that if $E+D \leq \varepsilon$, then there exists $R \in(3,4)$ such that if $\Phi$ solves,

$$
\Delta \Phi=c \text { in } B_{R} \quad \text { and } \quad \nu \cdot \nabla \Phi=\nu \cdot \bar{j} \quad \text { on } \partial B_{R},
$$


then

$$
\int_{B_{2}} \int_{0}^{1} \frac{1}{\rho}|j-\rho \nabla \Phi|^{2} \leq \tau E+C D
$$

Moreover,

$$
\sup _{B_{2}}\left|\nabla^{2} \Phi\right|^{2}+\sup _{B_{2}}|\nabla \Phi|^{2} \lesssim E+D
$$

To ease notation, let $f:=\nu \cdot j$ and $\bar{f}:=\int_{0}^{1} f=\nu \cdot \bar{j}$. Notice that $(\rho, j)$ solves the local version of (1.6):

$$
\begin{cases}\partial_{t} \rho+\nabla \cdot j=0 & \text { in } B_{R} \\ \rho_{0}=\mu, \rho_{1}=\lambda & \text { in } B_{R} \\ \nu \cdot j=f & \text { on } \partial B_{R} \times(0,1)\end{cases}
$$

The strategy of the proof of Proposition 1.6 is the same as for [26, Prop. 3.3], namely to choose first a good radius $R$ for which the flux $\bar{f}$ is well behaved on $\partial B_{R}$ and then rely on an almost orthogonality property (see Lemma 1.7 below) in order to be able to use the minimization properties of $(\rho, j)$ and finally obtain the desired estimates through the construction of a competitor (see Lemma 1.8). However, each of these steps is considerably harder than in [26]. Indeed, since our analysis is at the $L^{2}$ level, to obtain $L^{2}$ bounds on $\nabla \Phi$, we would need $L^{2}$ bounds on $\bar{f}$ (or at least $L^{\frac{2(d-1)}{d}}$ bounds by the Sobolev trace embedding). However since in general $j$ is just a measure, there is no hope in this setting to obtain such bounds. In order to solve this issue, we first prove an analog of Proposition 1.6 but with $\Phi$ replaced by $\phi$, which is a solution of

$$
\Delta \phi=c \text { in } B_{R} \quad \text { and } \quad \nu \cdot \nabla \phi=\hat{g} \text { on } \partial B_{R},
$$

where $\hat{g}$ is a regularization through rearrangement of $\bar{f}$ with good $L^{2}$ bounds. More specifically, treating separately incoming and outgoing fluxes $\bar{f}_{ \pm}$(so that $\bar{f}=\bar{f}_{+}-\bar{f}_{-}$ and similarly $\hat{g}=\hat{g}_{+}-\hat{g}_{-}$), we construct in Lemma 3.2 densities $\hat{g}_{ \pm}$on $\partial B_{R}$ with (we refer to 2.25) for the definition of $W_{\partial B_{R}}$ )

$$
\int_{\partial B_{R}} \hat{g}_{ \pm}^{2} \lesssim E+D \quad \text { and } \quad W_{\partial B_{R}}^{2}\left(\bar{f}_{ \pm}, \hat{g}_{ \pm}\right) \lesssim(E+D)^{\frac{d+3}{d+2}}
$$

In order to construct $\hat{g}_{ \pm}$, we consider the time dependent version of the Lagrangian problem and couple it with the data to the effect of having trajectories connecting the constant density $\kappa_{\mu}$ at time $t=-1$, the measure $\mu$ at time $t=0$, the measure $\lambda$ at time $t=1$ and finally the constant density $\kappa_{\lambda}$ at time $t=2$ (see Figure 1). Since $E+D \ll 1$, thanks to the $L^{\infty}$ bound on the displacement proven in Lemma 2.9 , particles hitting $\partial B_{R} \times(0,1)$ must come from (respectively end in) a small neighborhood of $\partial B_{R}$ at time $t=-1$ (respectively at time $t=2$ ). A key point in deriving 1.22 ) is that at time $t=-1$ and $t=2$, the densities are well-behaved (being constant) and thus the number of particles coming from such a small neighborhood is under control. 


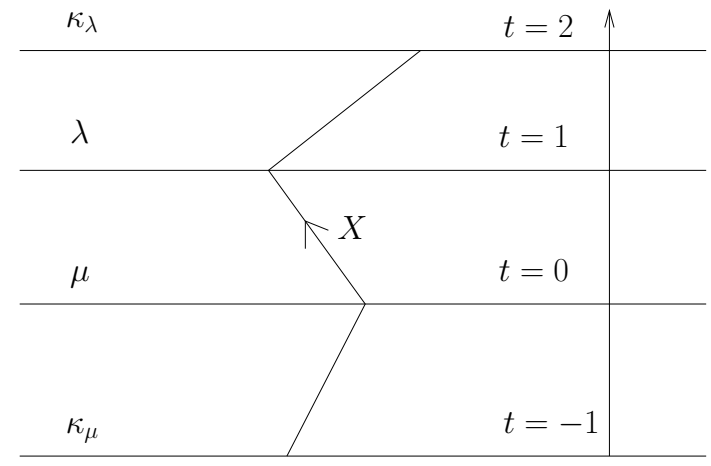

Figure 1: The trajectories $X$.

Once the good radius $R$ is chosen, the second step in the proof of Proposition 1.6 is the following approximate orthogonality result (cf. [26, Rem. 3.4] for an explanation of the name).

Lemma 1.7 (Orthogonality). For every $0<r \ll 1$, there exist $\varepsilon(r)>0$ and $C(r)>0$ such that if $E+D \leq \varepsilon$,

$$
\int_{B_{2}} \int_{0}^{1} \frac{1}{\rho}|j-\rho \nabla \phi|^{2}-\left(\int_{B_{R}} \int_{0}^{1} \frac{1}{\rho}|j|^{2}-\int_{B_{R}}|\nabla \phi|^{2}\right) \leq r E+C D .
$$

In order to prove Lemma 1.7, we consider $\hat{g}_{r}$ the convolution at scale $r$ (on $\partial B_{R}$ ) of $\hat{g}$ and show that for $\phi^{r}$ the solution of (1.21) but with the (further) regularized flux $\hat{g}_{r}$ instead of $\hat{g}$,

$$
\begin{aligned}
\int_{B_{R}} \int_{0}^{1} \frac{1}{\rho} \mid j & -\left.\rho \nabla \phi^{r}\right|^{2}=\int_{B_{R}} \int_{0}^{1} \frac{1}{\rho}|j|^{2}-\int_{B_{R}}\left|\nabla \phi^{r}\right|^{2} \\
& +2 \int_{B_{R}} \phi^{r} d(\mu-\lambda)+2 \int_{\partial B_{R}} \phi^{r} d\left(\hat{g}_{r}-\bar{f}\right)+\int_{B_{R}} \int_{0}^{1}\left|\nabla \phi^{r}\right|^{2}(d \rho-d x)
\end{aligned}
$$

and estimate the three error terms in the second line. The first term is estimated using that $\mu$ and $\lambda$ are very close in Wasserstein distance. The second term is estimated using the second part of $(1.22)$. While in [26, the last error term was easily estimated since in that case $\rho \leq 1$ (up to a small error) by McCann's displacement convexity, we need here a more delicate argument based on elliptic regularity and the fact that

$$
W_{B_{R}}^{2}\left(\int_{0}^{1} \rho, \kappa\right) \lesssim E+D
$$

This estimate is a consequence of a restriction result for the Wasserstein distance (see Lemma 2.10). It is here that we need the further regularization $\hat{g}_{r}$ of $\hat{g}$. The proof of 1.23$)$ is concluded using that $\nabla \phi$ and $\nabla \phi^{r}$ are very close in $B_{2}$.

In the third step we construct a competitor for the variational problem solved by $(\rho, j)$ : 
Lemma 1.8. For every $0<\tau \ll 1$, there exist $\varepsilon(\tau)>0$ and $C(\tau)>0$ such that if $E+D \leq \varepsilon$, then there exists a density-flux pair $(\widetilde{\rho}, \widetilde{j})$ satisfying 1.20 and such that

$$
\iint_{0}^{1} \frac{1}{\widetilde{\rho}}|\widetilde{j}|^{2}-\int_{B_{R}}|\nabla \phi|^{2} \leq \tau E+C D .
$$

Choosing $r=\tau$, Lemma 1.8 together with 1.23 and the fact that $(\rho, j)$ is minimizing concludes the proof of 1.18) (with $\nabla \phi$ instead of $\nabla \Phi)$.

To show Lemma 1.8 we introduce a small time like parameter $0<\tau \ll 1$ and work separately in the initial layer $(0, \tau)$, in $(\tau, 1-\tau)$ and in the final layer $(1-\tau, 1)$ (this can again be compared with the approach of $[6,28])$. We need to separately treat a small set of exceptional trajectories which either enter $B_{R}$ too early or exit too late. Even though this leads to some non trivial complications (see Section 3.1.3), we will ignore this issue here to keep the discussion simpler and assume that the flux $f$ is supported in $\partial B_{R} \times(3 \tau, 1-3 \tau)$. Using that the data term $D$ is small, we connect the measure $\mu$ to a constant $\kappa_{\mu}$ in $(0, \tau)$ and another constant $\kappa_{\lambda}$ to $\lambda$ in $(1-\tau, 1)$. The leading order construction in $B_{R} \times(\tau, 1-\tau)$ is given by

$$
\widetilde{\rho}_{t}:=\frac{t-\tau}{1-2 \tau} \kappa_{\lambda}+\frac{1-\tau-t}{1-2 \tau} \kappa_{\mu}, \quad \widetilde{j}_{t}:=\frac{1}{1-2 \tau} \nabla \phi,
$$

which connect the two constants but has the flux boundary condition $\hat{g}$ instead of $f$. To correct the boundary condition, we first make a construction in the boundary layer $B_{R} \backslash \bar{B}_{R-r} \times(\tau, 1-\tau)$ with $0<r \ll 1$, using [26, Lem. 2.4] to connect the constant-in- $t$ density $\hat{g}$ to

$$
\widetilde{g}:= \begin{cases}\frac{1}{\tau} \hat{g}_{+} & \text {for } t \in(\tau, 2 \tau) \\ -\frac{1}{\tau} \hat{g}_{-} & \text {for } t \in(1-2 \tau, 1-\tau) .\end{cases}
$$

Since $\widetilde{g}$ is supported in $(\tau, 2 \tau) \cup(1-2 \tau, 1-\tau)$ and $f$ is supported in $(3 \tau, 1-3 \tau)$, we can then use the second estimate in 1.22 to connnect $\widetilde{g}$ to $f$ in $\partial B_{R} \times[0,1]$ with cost $\frac{1}{\tau}(E+D)^{\frac{d+3}{d+2}}$ (see Lemma 3.5 .

In a a final step, we conclude the proof of (1.18) using that by elliptic regularity and (1.22), $\nabla \phi$ and $\nabla \Phi$ are very close in $B_{2}$,

\subsubsection{Proof of Theorem 1.2}

Let us recall that from now on, we assume that the target measure is the Lebesgue measure. The reason for this is that we will heavily use its invariance properties under shifts.

The first step in the proof of Theorem 1.2 is a Campanato iteration scheme based on Theorem 1.4. To state the result we need to fix some notation. For a sequence of approximately geometric radii i.e.

$$
\bar{R} \geq R_{0} \geq \cdots \geq R_{K} \geq C \quad \text { with } \quad R_{k-1} \geq 2 R_{k} \geq \frac{1}{C} R_{k-1}
$$


with $\bar{R} \sim R_{0}$ and $R_{K} \sim 1$, we set (recall 1.14 )

$$
E_{k}:=E\left(R_{k}\right)=\frac{1}{\left|B_{6 R_{k}}\right|} \int_{\left(B_{6 R_{k}} \times \mathbb{R}^{d}\right) \cup\left(\mathbb{R}^{d} \times B_{6 R_{k}}\right)}|x-y|^{2} d \pi_{k},
$$

where $\pi_{k}$ is the coupling recursively defined by $\pi_{0}:=\pi$ and

$$
\pi_{k}:=\left(\mathrm{id}, \mathrm{id}-\nabla \Phi_{k-1}(0)\right) \# \pi_{k-1},
$$

where $\Phi_{k}$ solves the Poisson equation

$$
\Delta \Phi_{k}=c \text { in } B_{R_{k}} \quad \text { and } \quad \nu \cdot \nabla \Phi_{k}=\nu \cdot \bar{j}_{k} \text { on } \partial B_{R_{k}} .
$$

The flux $j_{k}$ is defined as in (1.4) with $\pi_{k}$ playing the role of $\pi$ and $\bar{j}_{k}$ is then obtained by integrating in time.

Proposition 1.9. Assume that

$$
\begin{aligned}
\frac{1}{\left|B_{\bar{R}}\right|} \int_{\left(B_{\bar{R}} \times \mathbb{R}^{d}\right) \cup\left(\mathbb{R}^{d} \times B_{\bar{R}}\right)}|x-y|^{2} d \pi & \leq \beta(\bar{R}) \\
& \text { and } \quad \frac{1}{\left|B_{R}\right|} W_{B_{R}}^{2}(\mu, \kappa) \leq \beta(R) \quad \forall R \in[1, \bar{R}] .
\end{aligned}
$$

Then, there exists a sequence of approximately geometric radii $\left(R_{k}\right)_{0 \leq k \leq K}$ such that

$$
E_{k} \lesssim \beta\left(R_{k}\right)
$$

and

$$
\left|\nabla \Phi_{k}(0)\right|^{2} \lesssim \beta\left(R_{k}\right)
$$

Assuming that $\beta(R)=R^{\alpha}$, we can see that we are performing here a Campanato iteration at the $C^{0, \frac{\alpha}{2}}$ level for the displacement rather than at the $C^{1, \frac{\alpha}{2}}$ level as in [26]. In fact, we can run the iteration as long as $\frac{1}{R_{k}^{2}} E_{k}+\frac{1}{R_{k}^{2}} D\left(R_{k}\right) \ll 1$. For large scales $R_{k} \gg 1$ this holds for $\alpha<2$ (and notice that Proposition 1.9 actually would hold under this weaker assumption on $\alpha$ ), while for small scales $R_{k} \ll 1$ it would require $\alpha>2$ (and this would be inconsistent).

From (1.27) and the iterative definition 1.25), we obtain an estimate in the strong $L^{2}$ topology of the deviation of the displacement $y-x$ from the cumulated shift $\widetilde{h}:=\sum_{k=0}^{K-1} \nabla \Phi_{k}(0)$. The main ingredient to upgrade this into the statement of Theorem 1.2 is a better identification of the shift, taking into account cancellation effects. Indeed, a naive computation gives

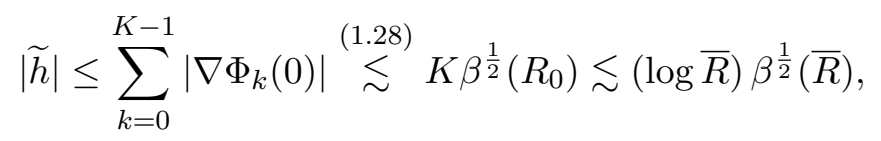

which fails by $\log \bar{R}$ the expected estimate $|\widetilde{h}| \lesssim \beta^{\frac{1}{2}}(\bar{R})$.

To state the identification result let $\left(R_{k}\right)_{k}$ be the sequence of radii from Proposition 1.9 and for each $k \in[0, K]$, let $u_{k}$ be the (distributional) solution to

$$
\Delta u_{k}=\mu-1 \text { in } B_{R_{k}} \quad \text { and } \quad \nu \cdot \nabla u_{k}=\nu \cdot \bar{j}_{k} \text { on } \partial B_{R_{k}} .
$$

Then we have 
Proposition 1.10. For every $k \in[0, K-1]$ and $R \in\left[R_{k+1}, R_{k}\right]$, we have the two estimates on the cumulative flux

$$
\left|\sum_{\ell=0}^{k} \nabla \Phi_{\ell}(0)-\frac{1}{\left|B_{R_{k}}\right|} \int_{\partial B_{R_{k}}} x \nu \cdot \nabla u_{0}\right| \lesssim \frac{\beta\left(R_{k}\right)}{R_{k}}
$$

and

$$
\left|\sum_{\ell=0}^{k-1} \nabla \Phi_{\ell}(0)+\int \eta_{R} \nabla u_{k}-\int \eta_{R} \nabla u_{0}\right| \lesssim \frac{\beta\left(R_{k}\right)}{R_{k}},
$$

where $\eta_{R}$ is as in 1.1 .

Since 1.32 is a fairly direct consequence of 1.31), we will just comment on the proof of the latter. For each $k \in[0, K]$ let $v_{k}$ be the distributional solution to

$$
\Delta v_{k}=\mu-1-c \text { in } B_{R_{k}} \quad \text { and } \quad \nu \cdot \nabla v_{k}=0 \text { on } \partial B_{R_{k}}
$$

so that $u_{k}=v_{k}+\Phi_{k}$. The first ingredient for the proof of (1.31) is the following estimate for fixed $k$.

Lemma 1.11. For every $k \in[1, K-1]$,

$$
\left|\nabla \Phi_{k}(0)-\frac{1}{\left|B_{R_{k}}\right|} \int_{\partial B_{R_{k}}} x \nu \cdot \nabla v_{k-1}\right| \lesssim \frac{\beta\left(R_{k}\right)}{R_{k}} .
$$

This result is in itself quite remarkable since it proves that the local shift $\nabla \Phi_{k}(0)$ depends (to leading order) only on the local structure of $\mu$ in $B_{R_{k-1}}$ (since $v_{k-1}$ only depends on $\mu\left\llcorner B_{R_{k-1}}\right)$.

Lemma 1.11 follows itself from the combination of two facts. The first is that the shift $\nabla \Phi_{k-1}(0)$, cf. 1.25), which is additive in the displacement retains an almost additive effect on the Eulerian flux:

Lemma 1.12. For every $k \in[1, K]$,

$$
\left|\frac{1}{\left|B_{R_{k}}\right|} \int_{B_{R_{k}}}\left(\bar{j}_{k}+\nabla \Phi_{k-1}(0)-\bar{j}_{k-1}\right)\right| \lesssim \frac{\beta\left(R_{k}\right)}{R_{k}} .
$$

This is proven using that $R_{k}$ is a good radius. The second, and more substantial result leading to Lemma 1.11 is a linearization result at the level of the Eulerian flux:

Lemma 1.13. Recall the definition (1.30) of $u_{k}$. For $R \in\left[R_{k+1}, R_{k}\right]$, there holds

$$
\begin{aligned}
\left|\int \eta_{R}\left(\bar{j}_{k}-\nabla u_{k}\right)\right| & \lesssim \frac{E_{k}}{R_{k}}, \\
\frac{1}{\left|B_{R_{k+1}}\right|}\left|\int_{B_{R_{k+1}}}\left(\bar{j}_{k}-\nabla u_{k}\right)\right| & \lesssim \frac{E_{k}}{R_{k}} .
\end{aligned}
$$


While 1.36$)$ is very important in combination with 1.32 to obtain estimate 11.11$)$, we are mainly interested in (1.37) for this discussion. Both estimates are obtained by proving that the divergence free vector field $\bar{j}_{k}-\nabla u_{k}$ is weakly close on large scale to a gradient field, namely the gradient of $\frac{1}{2}|y|^{2}-\psi_{k}-u_{k}$, where $\psi_{k}$ is the Kantorovich potential of $\pi_{k}$.

Since it is at the basis of our argument, let us derive Lemma 1.11 from Lemma 1.12 and Lemma 1.13

Proof of Lemma 1.11. The starting point is that since $\nabla \Phi_{k}$ is harmonic and thus satisfies the mean-value property and since, concentrating for instance on the first variable, $\nabla \cdot\left(x_{1} \nabla \Phi_{k}\right)=\partial_{1} \Phi_{k}+x_{1} c$, we have by the divergence theorem

$$
\nabla \Phi_{k}(0)=\frac{1}{\left|B_{R_{k}}\right|} \int_{B_{R_{k}}} \nabla \Phi_{k}=\frac{1}{\left|B_{R_{k}}\right|} \int_{\partial B_{R_{k}}} x \nu \cdot \nabla \Phi_{k} .
$$

Observe that by (1.6) $\nabla \cdot \bar{j}_{k}=\mu-1$ in $B_{R_{k}}$. Combining this with 1.33 implies that $\nabla \cdot\left(\bar{j}_{k}-\nabla v_{k-1}\right)=c$ in $B_{R_{k}}$. Hence, we have again by the divergence theorem

$$
\begin{aligned}
\nabla \Phi_{k}(0)-\frac{1}{\left|B_{R_{k}}\right|} \int_{\partial B_{R_{k}}} x \nu \cdot \nabla v_{k-1} & =\frac{1}{\left|B_{R_{k}}\right|} \int_{\partial B_{R_{k}}} x \nu \cdot\left(\nabla \Phi_{k}-\nabla v_{k-1}\right) \\
& \stackrel{1.26}{=} \frac{1}{\left|B_{R_{k}}\right|} \int_{\partial B_{R_{k}}} x \nu \cdot\left(\bar{j}_{k}-\nabla v_{k-1}\right) \\
& =\frac{1}{\left|B_{R_{k}}\right|} \int_{B_{R_{k}}}\left(\bar{j}_{k}-\nabla v_{k-1}\right) .
\end{aligned}
$$

Writing

$$
\bar{j}_{k}-\nabla v_{k-1}=\left(\bar{j}_{k}+\nabla \Phi_{k-1}(0)-\bar{j}_{k-1}\right)+\left(\bar{j}_{k-1}-\nabla \Phi_{k-1}(0)-\nabla v_{k-1}\right),
$$

using 1.38 for $\nabla \Phi_{k-1}$ and the fact that $u_{k-1}=\Phi_{k-1}+v_{k-1}$, we obtain as desired

$$
\begin{aligned}
& \left|\nabla \Phi_{k}(0)-\frac{1}{\left|B_{R_{k}}\right|} \int_{\partial B_{R_{k}}} x \nu \cdot \nabla v_{k-1}\right| \\
& \leq\left|\frac{1}{\left|B_{R_{k}}\right|} \int_{B_{R_{k}}}\left(\bar{j}_{k}+\nabla \Phi_{k-1}(0)-\bar{j}_{k-1}\right)\right|+\left|\frac{1}{\left|B_{R_{k}}\right|} \int_{B_{R_{k}}}\left(\bar{j}_{k-1}-\nabla u_{k-1}\right)\right| \\
& \\
& \underset{\frac{1.37}{\lesssim} \frac{\beta\left(R_{k}\right)}{R_{k}}+\frac{E_{k-1}}{R_{k-1}}}{\lesssim \frac{\beta\left(R_{k}\right)}{R_{k}} .}
\end{aligned}
$$

Besides Lemma 1.11, the second main ingredient for the proof of (1.31) is a summation formula for the fluxes of $v_{k}$. 
Lemma 1.14. For every $k \in[1, K]$,

$$
\frac{1}{\left|B_{R_{k}}\right|} \int_{\partial B_{R_{k}}} x \nu \cdot \nabla v_{0}=\sum_{\ell=1}^{k} \frac{1}{\left|B_{R_{\ell}}\right|} \int_{\partial B_{R_{\ell}}} x \nu \cdot \nabla v_{\ell-1} .
$$

The proof of (1.39) is based on the fact that each $v_{k}$ type of solves the same equation (see $(1.33)$ ) and that we may find an explicit formula relating the flux $\frac{1}{\left|B_{R_{k}}\right|} \int_{\partial B_{R_{k}}} x \nu$. $\nabla v_{k-1}$ and the measure $\mu$.

Let us point out that (1.31) is a direct consequence of (1.34), 1.39 and hypothesis (1.7) on $\beta$, once observed that by (1.38), and since $u_{0}=v_{0}+\Phi_{0}$,

$$
\nabla \Phi_{0}(0)-\frac{1}{\left|B_{R_{k}}\right|} \int_{\partial B_{R_{k}}} x \nu \cdot \nabla u_{0}=-\frac{1}{\left|B_{R_{k}}\right|} \int_{\partial B_{R_{k}}} x \nu \cdot \nabla v_{0}
$$

\subsection{Comparison with the first part of [25]}

We close this introduction by stressing the connection and differences between this paper and [25]. This paper replaces and supersedes the first (deterministic) part of [25] (which will thus not be submitted anywhere). The second part containing the application to the optimal matching problem will appear elsewhere. A first main difference with 25 is that we are able here to directly relate the displacements to the flux given by the Poisson equation (see (1.11) ) and thus confirming the linearization ansatz by Caracciolo and al. from [17]. As a by-product, we obtain optimal estimates for the shift $h$ in $(1.12$ while [25, Th. 1.1] was only optimal up to a logarithmic factor in the system size i.e $\left|h_{R}\right| \lesssim \log ^{\frac{3}{2}} \bar{R}$ in dimension $d=2$ and $\left|h_{R}\right| \lesssim \log \bar{R}$ in higher dimension (see (1.29) ). Another main difference is in the statement and proofs of the harmonic approximation result (see Theorem 1.4). Compared with [25, Prop. 1.5], where the source was given by the Lebesgue measure, it is stated here for general source and target measures. While this might look like a minor point, it actually means that we cannot rely at all on McCann's interpolation convexity estimate to obtain an $L^{\infty}$ bound on the Eulerian density $\rho$ (since in general it is only a measure), see for instance [25, Lem.2.4]. Having to go around this relatively rigid argument actually led to a completely revised, simpler and hopefully more flexible proof. Let us point out that having to connect arbitrary source and target measures also called for a new proof of the $L^{\infty}$ bound on the displacement (compare the proofs of Lemma 2.9 and [26, Lem. 4.1]). A last difference, more on the technical side, is that we substantially simplified the Camapanato iteration with respect to [25, Th. 1.1].

\section{Acknowledgements}

MH gratefully acknowledges partial support by the DFG through the CRC 1060 "The Mathematics of Emerging Effects" and by the Hausdorff Center for Mathematics during the first part of this project. During the second part of this work MH has been funded by the Vienna Science and Technology Fund (WWTF) through project 
VRG17-005. In the final part MH has been funded by the Deutsche Forschungsgemeinschaft (DFG, German Research Foundation) under Germany's Excellence Strategy EXC 2044 -390685587, Mathematics Münster: Dynamics-Geometry-Structure MG acknowledges partial support from the ANR project SHAPO. MG and MH thank the Max Planck Institute MIS for its warm hospitality.

\section{Preliminaries}

\section{$2.1 \quad$ Notation}

In this paper we will use the following notation. We recall from the introduction that the symbols $\sim, \gtrsim, \lesssim$ indicate estimates that hold up to a global constant $C$, which typically only depends on the dimension $d$. For instance, $f \lesssim g$ means that there exists such a constant with $f \leq C g, f \sim g$ means $f \lesssim g$ and $g \lesssim f$. An assumption of the form $f \ll 1$ means that there exists $\varepsilon>0$, typically only depending on dimension, such that if $f \leq \varepsilon$, then the conclusion holds. We write log for the natural logarithm. We denote by $\mathcal{H}^{k}$ the $k$-dimensional Hausdorff measure. For a set $E, \nu_{E}$ will always denote the external normal to $E$. When clear from the context we will drop the explicit dependence on the set. We write $|E|$ for the Lebesgue measure of a set $E$ and $I(E)$ for the indicator function of $E$. When no confusion is possible, we will drop the integration measures in the integrals as well as the domain of integration. Similarly, we will often identify, if possible, measures with their densities with respect to the Lebesgue measure. For $R>0$ and $x_{0} \in \mathbb{R}^{d}, B_{R}\left(x_{0}\right)$ denotes the ball of radius $R$ centered in $x_{0}$. When $x_{0}=0$, we will simply write $B_{R}$ for $B_{R}(0)$. We denote the gradient on $\partial B_{R}$ by $\nabla_{\tan }$. For a measure $\rho$ on $\mathbb{R}^{d} \times[0,1]$, we denote by $\bar{\rho}$ its time integral i.e., the measure defined for $\zeta \in C_{c}^{0}\left(\mathbb{R}^{d}\right)$ as

$$
\int_{\mathbb{R}^{d}} \zeta d \bar{\rho}=\int_{\mathbb{R}^{d}} \int_{0}^{1} \zeta d \rho .
$$

From now on, we use the letter $\zeta$ for a generic real-valued test function and $\xi$ for a generic vector-valued test function, that is $\xi \in C_{c}^{0}\left(\mathbb{R}^{d}\right)^{d}$. For a given radius $R>0$, we let $\Pi_{R}(x):=R \frac{x}{|x|}$ be the projection on $\partial B_{R}$ and define for every measure $\rho$ on $\mathbb{R}^{d}$, the projected measure on $\partial B_{R}, \hat{\rho}:=\Pi_{R} \# \rho$, that is

$$
\int \zeta d \hat{\rho}=\int \zeta\left(R \frac{x}{|x|}\right) d \rho(x)
$$

For given $f$ and $g$ (potentially measures), we let $c$ be the generic constant such that the Poisson equation

$$
\Delta \varphi=g+c \text { in } B_{R} \quad \text { and } \quad \nu \cdot \nabla \varphi=f \text { on } \partial B_{R}
$$

is solvable i.e

$$
c=\frac{1}{\left|B_{R}\right|}\left(\int_{\partial B_{R}} d f-\int_{B_{R}} d g\right)
$$


We use $W^{2}$ for the squared 2-Wasserstein distance (see Section 2.3 for more details). For a (positive) measure $\mu$ and a set $O \subset \mathbb{R}^{d}$, we define $\kappa:=\frac{\mu(O)}{|O|}$ the generic constant such that

$$
W_{O}^{2}(\mu, \kappa):=W^{2}(\mu\llcorner O, \kappa d x\llcorner O)
$$

is finite. For $\zeta$ a function on $\mathbb{R}^{d} \times \mathbb{R}$ and $t \in \mathbb{R}$, we will often use the short-hand notation $\zeta_{t}:=\zeta(\cdot, t)$.

\section{$2.2 \quad$ Elliptic estimates}

We gather in this section a few technical lemmas giving more or less standard estimates for solutions of the Poisson equation.

Lemma 2.1. Let $R \in(3,4)$ and $0 \leq r \ll 1$ be fixed. Letting $f_{r}$ denote the convolution at scale $r$ on $\partial B_{R}$ (with the convention that $f_{0}=f$ ) and then $\varphi^{r}$ with $\int_{B_{R}} \varphi^{r}=0$ be the solution of

$$
\Delta \varphi^{r}=c \quad \text { in } B_{R} \quad \text { and } \quad \nu \cdot \nabla \varphi^{r}=f_{r} \quad \text { on } \partial B_{R} .
$$

Then $\varphi^{r}$ is regular up to the boundary with

$$
r^{2} \sup _{B_{R}}\left|\nabla^{3} \varphi^{r}\right|+r \sup _{B_{R}}\left|\nabla^{2} \varphi^{r}\right|+\sup _{B_{R}}\left|\nabla \varphi^{r}\right| \lesssim\left(\frac{1}{r^{d-1}} \int_{\partial B_{R}} f^{2}\right)^{\frac{1}{2}},
$$

is close to $\varphi$ in the interior

$$
\sup _{B_{2}}\left|\nabla\left(\varphi^{r}-\varphi\right)\right| \lesssim r\left(\int_{\partial B_{R}} f^{2}\right)^{\frac{1}{2}}
$$

and satisfies the uniform-in-r regularity estimates both up to the boundary

$$
\left(\int_{\partial B_{s}}\left|\nabla \varphi^{r}\right|^{2}\right)^{\frac{1}{2}} \lesssim\left(\int_{\partial B_{R}} f_{r}^{2}\right)^{\frac{1}{2}} \quad \forall 0<s \leq R,
$$

and in the interior

$$
\sup _{B_{2}}\left|\nabla^{2} \varphi^{r}\right|+\sup _{B_{2}}\left|\nabla \varphi^{r}\right| \lesssim\left(\int_{B_{R}}\left|\nabla \varphi^{r}\right|^{2}\right)^{\frac{1}{2}} \lesssim\left(\int_{\partial B_{R}} f_{r}^{2}\right)^{\frac{1}{2}} .
$$

Proof. A proof of (2.6) and (2.7) can be found for instance in [26, Lem. 2.1], so we are left to show (2.4) and (2.5).

Up to subtracting a quadratic function to $\varphi^{r}$, we may assume without loss of generality that $c=0$ (and thus $\varphi^{r}$ is harmonic in $B_{R}$ ). We start by the argument for (2.4) and restrict to the (more involved) estimate on $\nabla^{3} \varphi^{r}$. By the maximum principle for the component-wise harmonic $\nabla^{3} \varphi^{r}$, it is enough to establish

$$
r^{2} \sup _{\partial B_{R}}\left|\nabla^{3} \varphi^{r}\right| \lesssim\left(\frac{1}{r^{d-1}} \int_{\partial B_{R}} f^{2}\right)^{\frac{1}{2}}
$$


Fixing an integer $n>\frac{d-1}{2}$, we first claim that for $r \in(0,1]$,

$$
\sup _{\partial B_{R}}|\zeta| \lesssim r^{n}\left(\frac{1}{r^{d-1}} \int_{\partial B_{R}}\left|\nabla_{t a n}^{n} \zeta\right|^{2}\right)^{\frac{1}{2}}+\left(\frac{1}{r^{d-1}} \int_{\partial B_{R}}|\zeta|^{2}\right)^{\frac{1}{2}} .
$$

To prove (2.8), we start with the standard Sobolev embedding on $\mathbb{R}^{d-1}, \sup _{\mathbb{R}^{d-1}}|\zeta|^{2} \lesssim$ $\int_{\mathbb{R}^{d}}\left(\left|\nabla^{n} \zeta\right|^{2}+\zeta^{2}\right)$, which by scaling turns into

$$
\sup _{\mathbb{R}^{d-1}}|\zeta|^{2} \lesssim r^{2 n-(d-1)} \int_{\mathbb{R}^{d}}\left|\nabla^{n} \zeta\right|^{2}+\frac{1}{r^{d-1}} \int_{\mathbb{R}^{d-1}} \zeta^{2} .
$$

This can easily be localized (with help of an extension operator) to

$$
\sup _{[0,1]^{d-1}}|\zeta|^{2} \lesssim r^{2 n-(d-1)} \int_{[0,1]^{d-1}}\left|\nabla^{n} \zeta\right|^{2}+\frac{1}{r^{d-1}} \int_{[0,1]^{d-1}} \zeta^{2}
$$

Covering $\partial B_{R}$ by finitely many patches that are diffeomorphic to $[0,1]^{d-1}$, we conclude the proof of 2.8 .

Applying 2.8 to $\zeta=\nabla^{3} \varphi^{r}$, we are left with the proof of

$$
r^{n+2}\left(\int_{\partial B_{R}}\left|\nabla_{t a n}^{n} \nabla^{3} \varphi^{r}\right|^{2}\right)^{\frac{1}{2}}+r^{2}\left(\int_{\partial B_{R}}\left|\nabla^{3} \varphi^{r}\right|^{2}\right)^{\frac{1}{2}} \lesssim\left(\int_{\partial B_{R}} f^{2}\right)^{\frac{1}{2}} .
$$

This is a direct consequence of the estimate

$$
\int_{\partial B_{R}}\left|\nabla_{t a n}^{m} \nabla^{3} \varphi^{r}\right|^{2} \lesssim \int_{\partial B_{R}}\left|\nabla_{t a n}^{m+2} f_{r}\right|^{2}
$$

which will be shown to hold for every $m \in \mathbb{N}$, and the fact that

$$
r^{m+2}\left(\int_{\partial B_{R}}\left|\nabla_{t a n}^{m+2} f_{r}\right|^{2}\right)^{\frac{1}{2}} \lesssim\left(\int_{\partial B_{R}} f^{2}\right)^{\frac{1}{2}}
$$

which follows from the properties of the convolution.

Let us prove (2.9) and fix $m \in \mathbb{N}$. By Pohozaev identity (see [21, Lem. 8.3.2]) we have for every harmonic function $\zeta$ in $B_{R}$,

$$
\int_{\partial B_{R}}|\nabla \zeta|^{2} \lesssim \int_{\partial B_{R}}\left|\nabla_{\tan } \zeta\right|^{2} \lesssim \int_{\partial B_{R}}|\nu \cdot \nabla \zeta|^{2}
$$

In particular, the Dirichlet-to-Neumann map $\zeta \mapsto \nabla \zeta$ is bounded from $H^{1}\left(\partial B_{R}\right)$ into $L^{2}\left(\partial B_{R}\right)$. As the map commutes with rotations, it also commutes with their infinitesimal generators $\left\{x_{i} \partial_{j}-x_{j} \partial_{i}\right\}_{i \neq j=1, \cdots, d}$, which in turn span the tangent space of $\partial B_{R}$. Hence, for $k \in \mathbb{N}$, the map is also bounded from $H^{k+1}\left(\partial B_{R}\right)$ to $H^{k}\left(\partial B_{R}\right)$. Applying this first to $\zeta=\nabla^{2} \varphi^{r}$ and $k=m$ and then to $\zeta=\nabla \varphi^{r}$ and $k=m+1$, we get

$$
\int_{\partial B_{R}}\left|\nabla_{t a n}^{m} \nabla^{3} \varphi^{r}\right|^{2} \lesssim \int_{\partial B_{R}}\left|\nabla_{t a n}^{m+2} \nabla \varphi^{r}\right|^{2}
$$


Arguing as above we obtain that also the Neumann-to-Dirichlet map $\nu \cdot \nabla \zeta \rightarrow \zeta$ is bounded from $H^{k}\left(\partial B_{R}\right)$ to $H^{k+1}\left(\partial B_{R}\right)$ for $k \in \mathbb{N}$. Applying this to $\zeta=\varphi^{r}$ and $k=m+2$, we conclude the proof of $(2.9)$.

We finally prove (2.5). By interior regularity for the harmonic $\varphi^{r}-\varphi$, we have

$$
\sup _{B_{2}}\left|\nabla\left(\varphi^{r}-\varphi\right)\right| \lesssim\left(\int_{\partial B_{R}}\left(\varphi^{r}-\varphi\right)^{2}\right)^{\frac{1}{2}} \lesssim r\left(\int_{\partial B_{R}}\left|\nabla_{\tan } \varphi\right|^{2}\right)^{\frac{1}{2}} \stackrel{2.10}{\lesssim} r\left(\int_{\partial B_{R}}|f|^{2}\right)^{\frac{1}{2}},
$$

where in the last inequality we used that $\varphi$ is harmonic to apply (2.10).

Lemma 2.2. For $R>0$, recall that $\Pi_{R}(x)=R \frac{x}{|x|}$. For every $(f, g)$, with $g \geq 0$ and Spt $g \subset \bar{B}_{R} \backslash B_{\frac{R}{2}}$, letting $\hat{g}:=\Pi_{R} \# g$ (see $(2.2)$ ), the unique solution $\varphi$ (up to additive constants) of

$$
\Delta \varphi=g+c \text { in } B_{R} \quad \text { and } \quad \nu \cdot \nabla \varphi=f \text { on } \partial B_{R}
$$

satisfies

$$
\int_{B_{R}}|\nabla \varphi|^{2} \lesssim R\left(\int_{\partial B_{R}} f^{2}+\int_{\partial B_{R}} \hat{g}^{2}\right)
$$

Proof. By scaling, we may assume that $R=1$. Without loss of generality, we may also assume that $\int_{B_{1}} \varphi=0$ and that $\int_{\partial B_{1}} f^{2}+\int_{\partial B_{1}} \hat{g}^{2}<\infty$, otherwise there is nothing to prove. Using integration by parts, the trace inequality for Sobolev functions together with the Poincaré inequality for functions of mean zero,

$$
\begin{aligned}
\int_{B_{1}}|\nabla \varphi|^{2} & =\int_{\partial B_{1}} \varphi f-\int_{B_{1}} \varphi \Delta \varphi \\
& \leq\left(\int_{\partial B_{1}} \varphi^{2}\right)^{\frac{1}{2}}\left(\int_{\partial B_{1}} f^{2}\right)^{\frac{1}{2}}+\left|\int_{B_{1}} g \varphi\right| \\
& \lesssim\left(\int_{B_{1}}|\nabla \varphi|^{2}\right)^{\frac{1}{2}}\left(\int_{\partial B_{1}} f^{2}\right)^{\frac{1}{2}}+\left|\int_{B_{1}} g \varphi\right| .
\end{aligned}
$$

Hence, it is now enough to prove that

$$
\left|\int_{B_{1}} g \varphi\right| \lesssim\left(\int_{B_{1}}|\nabla \varphi|^{2}\right)^{\frac{1}{2}}\left(\int_{\partial B_{1}} \hat{g}^{2}\right)^{\frac{1}{2}} .
$$

Using that by hypothesis Spt $g \subset \bar{B}_{1} \backslash B_{\frac{1}{2}}$, we can write by definition 2.2

$$
\begin{aligned}
\left|\int_{B_{1}} g \varphi\right| & \leq\left|\int_{\partial B_{1}} \varphi \hat{g}\right|+\left|\int_{\partial B_{1}} \int_{\frac{1}{2}}^{1}(\varphi(r \omega)-\varphi(\omega)) g(r \omega) r^{d-1} d r d \omega\right| \\
& \lesssim\left(\int_{\partial B_{1}} \varphi^{2}\right)^{\frac{1}{2}}\left(\int_{\partial B_{1}} \hat{g}^{2}\right)^{\frac{1}{2}}+\int_{\partial B_{1}} \int_{\frac{1}{2}}^{1}|\varphi(r \omega)-\varphi(\omega)| g(r \omega) r^{d-1} d r d \omega \\
& \lesssim\left(\int_{B_{1}}|\nabla \varphi|^{2}\right)^{\frac{1}{2}}\left(\int_{\partial B_{1}} \hat{g}^{2}\right)^{\frac{1}{2}}+\int_{\partial B_{1}} \int_{\frac{1}{2}}^{1}|\varphi(r \omega)-\varphi(\omega)| g(r \omega) r^{d-1} d r d \omega
\end{aligned}
$$


where in the last line we used once more that $\int_{\partial B_{1}} \varphi^{2} \lesssim \int_{B_{1}}|\nabla \varphi|^{2}$. Since for $r \in\left(\frac{1}{2}, 1\right)$

$$
|\varphi(r \omega)-\varphi(\omega)| \leq\left(\int_{\frac{1}{2}}^{1}\left|\partial_{r} \varphi(s \omega)\right|^{2} d s\right)^{\frac{1}{2}} \lesssim\left(\int_{0}^{1}\left|\partial_{r} \varphi(s \omega)\right|^{2} s^{d-1} d s\right)^{\frac{1}{2}}
$$

estimate 2.12 follows from

$$
\begin{aligned}
& \int_{\partial B_{1}} \int_{\frac{1}{2}}^{1}|\varphi(r \omega)-\varphi(\omega)| g(r \omega) r^{d-1} d r d \omega \\
& \lesssim \int_{\partial B_{1}} \int_{\frac{1}{2}}^{1}\left(\int_{0}^{1}\left|\partial_{r} \varphi(s \omega)\right|^{2} s^{d-1} d s\right)^{\frac{1}{2}} g(r \omega) r^{d-1} d r d \omega \\
& \stackrel{2.2}{=} \int_{\partial B_{1}}\left(\int_{0}^{1}\left|\partial_{r} \varphi(s \omega)\right|^{2} s^{d-1} d s\right)^{\frac{1}{2}} \hat{g}(\omega) d \omega \\
& \leq\left(\int_{\partial B_{1}} \int_{0}^{1}\left|\partial_{r} \varphi(s \omega)\right|^{2} s^{d-1} d s\right)^{\frac{1}{2}}\left(\int_{\partial B_{1}} \hat{g}^{2}\right)^{\frac{1}{2}} .
\end{aligned}
$$

Lemma 2.2 will often be used in combination with the following elementary estimate.

Lemma 2.3. For $R>0$, recall that $\Pi_{R}(x)=R \frac{x}{|x|}$ denotes the projection on $\partial B_{R}$. There exists $1 \geq \varepsilon(d)>0$ (depending only on the dimension) such that for every $g \geq 0$ with Spt $g \subset B_{(1+\varepsilon) R} \backslash B_{(1-\varepsilon) R}$, setting $\hat{g}:=\Pi_{R} \# g$ (see 2.2)), we have

$$
R^{1-d}\left(\int g\right)^{2} \lesssim \int_{\partial B_{R}} \hat{g}^{2} \lesssim \sup g \int|R-| x|| g
$$

Proof. By scaling we may assume that $R=\sup g=1$. The first inequality is a direct consequence of Cauchy-Schwarz inequality in the form

$$
\left(\int g\right)^{2}=\left(\int_{\partial B_{1}} \hat{g}\right)^{2} \lesssim \int_{\partial B_{1}} \hat{g}^{2}
$$

We now turn to the second inequality. Let us first point out that if $\varepsilon \ll 1$, we have $\sup _{\partial B_{1}}|\hat{g}| \ll 1$ since Spt $g \subset B_{1+\varepsilon} \backslash B_{1-\varepsilon}$. Momentarily fixing $\omega \in \partial B_{1}$ and setting $\psi(r):=r^{d-1} g(r \omega)$ for $r>0$, we have $0 \leq \psi \leq(1+\varepsilon)^{d-1} \leq 2$ and

$$
\int_{0}^{\infty} \psi=\hat{g}(\omega)
$$

so that for $\omega \in \partial B_{1}$,

$$
\int_{0}^{\infty}|1-r| r^{d-1} g(r \omega) \geq \min _{\substack{0 \leq \tilde{\psi} \leq 2 \\ \int \tilde{\psi}=\hat{g}(\omega)}} \int_{0}^{\infty}|1-r| \widetilde{\psi}(r) \gtrsim \hat{g}(\omega),
$$


where the last inequality follows since the minimizer of

$$
\min _{\substack{0 \leq \tilde{\psi} \leq 2 \\ \int \tilde{\psi}=\hat{g}(\omega)}} \int_{0}^{\infty}|1-r| \widetilde{\psi}(r)
$$

is given by $2 I\left(|r-1| \leq \frac{1}{4} \hat{g}(\omega)\right)$ (which is admissible since $|\hat{g}(\omega)| \ll 1$ ).

Lemma 2.4. For every $R \in(3,4)$, every positive measures $f$ and $g$ on $\partial B_{R}$ with $f\left(\partial B_{R}\right)=g\left(\partial B_{R}\right)$ and every $0<\alpha<1$, the solution $\varphi$ with $\int_{B_{R}} \varphi=0$ of

$$
\Delta \varphi=0 \quad \text { in } B_{R} \quad \text { and } \quad \nu \cdot \nabla \varphi=f-g \text { on } \partial B_{R}
$$

satisfies

$$
\sup _{B_{2}}\left|\nabla^{2} \varphi\right|+\sup _{B_{2}}|\nabla \varphi| \lesssim W_{\partial B_{R}}^{\alpha}(f, g)\left[g\left(\partial B_{R}\right)\right]^{1-\frac{\alpha}{2}},
$$

where the implicit constant depends on $\alpha$.

Proof. Let $1<p<\frac{d}{d-1}$ be defined by $\frac{1}{p}=\frac{\alpha}{d}+\frac{d-1}{d}$. By the mean value formula,

$$
\sup _{B_{2}}\left|\nabla^{2} \varphi\right|+\sup _{B_{2}}|\nabla \varphi| \lesssim\left(\int_{B_{R}}|\nabla \varphi|^{p}\right)^{\frac{1}{p}}
$$

and it is therefore enough to prove that

$$
\left(\int_{B_{R}}|\nabla \varphi|^{p}\right)^{\frac{1}{p}} \lesssim W_{\partial B_{R}}^{\alpha}(f, g)\left[g\left(\partial B_{R}\right)\right]^{1-\frac{\alpha}{2}} .
$$

We prove this estimate by duality. Consider $q$ the dual exponent of $p$, i.e. $\frac{1}{p}+\frac{1}{q}=1$, and for arbitrary $\xi$ smooth and compactly supported in $B_{R}$ denote by $\omega$ the solution of the (dual) problem

$$
\Delta \omega=\nabla \cdot \xi \text { in } B_{R} \quad \text { and } \quad \nu \cdot \nabla \omega=0 \text { on } \partial B_{R}
$$

with $\int_{B_{R}} \omega=0$. Noting that $q>d$ and $\alpha=1-\frac{d}{q}$, Sobolev's embedding followed by Calderón-Zygmund estimates for the Neumann-Laplacian (see for instance [34, Th. $3.16])$ yields

$$
[\omega]_{\alpha, B_{R}} \lesssim\left(\int_{B_{R}}|\nabla \omega|^{q}\right)^{\frac{1}{q}} \lesssim\left(\int_{B_{R}}|\xi|^{q}\right)^{\frac{1}{q}} .
$$

Let $\pi$ be an optimal transference plan between $f$ and $g$. Using integration by parts 
we obtain

$$
\begin{aligned}
\int_{B_{R}} \nabla \varphi \cdot \xi=-\int_{B_{R}} \varphi \nabla \cdot \xi & =\int_{\partial B_{R}} \omega d(f-g) \\
& =\int_{\partial B_{R} \times \partial B_{R}}(\omega(x)-\omega(y)) d \pi(x, y) \\
& \lesssim[\omega]_{\alpha, B_{R}} \int_{\partial B_{R} \times \partial B_{R}}|x-y|^{\alpha} d \pi \\
& \stackrel{\text { Hölder }}{\lesssim}\left(\int_{B_{R}}|\xi|^{q}\right)^{\frac{1}{q}} W^{\alpha}(f, g)\left[g\left(\partial B_{R}\right)\right]^{1-\frac{\alpha}{2}} \\
& \lesssim\left(\int_{B_{R}}|\xi|^{q}\right)^{\frac{1}{q}} W_{\partial B_{R}}^{\alpha}(f, g)\left[g\left(\partial B_{R}\right)\right]^{1-\frac{\alpha}{2}} .
\end{aligned}
$$

This concludes the proof of 2.15 .

Arguing along the same lines, we may also prove:

Lemma 2.5. For $R>0$, let $\mu$ be a signed measure on $B_{R}$ and $f$ be a signed measure on $\partial B_{R}$, both with finite total variation. Then, if $\varphi$ solves

$$
\Delta \varphi=\mu \text { in } B_{R} \quad \text { and } \quad \nu \cdot \nabla \varphi=f \quad \text { on } \partial B_{R},
$$

we have $\nabla \varphi \in L^{p}\left(B_{R}\right)$ for each exponent $1 \leq p<\frac{d}{d-1}$.

\subsection{The optimal transport problem}

To fix notation, let us recall a few definitions and well-known facts about optimal transportation. Much more may be found for instance in the books [35, 31].

For a measure $\pi$ on $\mathbb{R}^{d} \times \mathbb{R}^{d}$, we denote its marginals by $\pi_{1}$ and $\pi_{2}$ i.e. $\pi_{1}(A)=$ $\pi\left(A \times \mathbb{R}^{d}\right)$ and $\pi_{2}(A)=\pi\left(\mathbb{R}^{d} \times A\right)$. For $\mu$ and $\lambda$ two positive measures with compact support and equal mass, we define the Wasserstein distance between $\mu$ and $\lambda$ as

$$
W^{2}(\mu, \lambda):=\min _{\pi_{1}=\mu, \pi_{2}=\lambda} \int|x-y|^{2} d \pi .
$$

Under our hypothesis, an optimal coupling always exists. By the Knott-Smith optimality criterion (see [35, Th. 2.12]), the coupling $\pi$ is optimal if and only if its support is cyclically monotone, i.e. there exists a convex lower semi-continuous function $\psi$ such that $\operatorname{Spt} \pi \subset \operatorname{Graph}(\partial \psi)$. Moreover, if $\mu$ does not give mass to Lebesgue negligible sets, by Brenier's Theorem (see [35, Th. 2.12] again), $\pi$ is optimal if and only if $\pi=(\mathrm{id} \times \nabla \psi) \# \mu$, where $\nabla \psi$ is the unique gradient of a convex function with $\nabla \psi \# \mu=\lambda$.

For $O \subset \mathbb{R}^{d}$, we denote $W_{O}(\mu, \lambda):=W(\mu\llcorner O, \lambda\llcorner O)$ and we recall that we let $\kappa$ be the generic constant such that $W_{O}(\mu, \kappa)$ is well-defined (see (2.3)). As in [26], a

\footnotetext{
${ }^{\text {ii here we identified the measure } \kappa d x \text { with } \kappa}$
} 
central point for our analysis is the Eulerian version of optimal transportation, also known as the Benamou-Brenier formulation (see for instance [35, Th. 8.1] or [4, Ch. $8])$. It states that

$$
W^{2}(\mu, \lambda)=\min _{(\rho, j)}\left\{\int_{\mathbb{R}^{d}} \int_{0}^{1} \frac{1}{\rho}|j|^{2}: \partial_{t} \rho+\nabla \cdot j=0, \rho_{0}=\mu \text { and } \rho_{1}=\lambda\right\},
$$

where the continuity equation and the boundary data are understood in the distributional sense, i.e. for every $\zeta \in C_{c}^{1}\left(\mathbb{R}^{d} \times[0,1]\right)$,

$$
\int_{\mathbb{R}^{d}} \int_{0}^{1} \partial_{t} \zeta d \rho+\nabla \zeta \cdot d j=\int_{\mathbb{R}^{d}} \zeta_{1} d \lambda-\int_{\mathbb{R}^{d}} \zeta_{0} d \mu
$$

(recall that $\left.\zeta_{t}=\zeta(\cdot, t)\right)$ and where

$$
\int_{\mathbb{R}^{d}} \int_{0}^{1} \frac{1}{\rho}|j|^{2}=\int_{\mathbb{R}^{d}} \int_{0}^{1}|v|^{2} d \rho
$$

if $j \ll \rho$ with $\frac{d j}{d \rho}=v$ and infinity otherwise (see [3, Th. 2.34]). Let us point out that in particular, the admissible measures for (2.18) are allowed to contain singular parts with respect to the Lebesgue measure. We also recall that for every $R>0$ we have the following dual definition of the Eulerian energy (see [31, Prop. 5.18])

$$
\frac{1}{2} \int_{B_{R}} \int_{0}^{1} \frac{1}{\rho}|j|^{2}=\sup _{\xi \in C_{c}^{0}\left(B_{R} \times[0,1]\right)^{d}} \int_{B_{R}} \int_{0}^{1} \xi \cdot d j-\frac{|\xi|^{2}}{2} d \rho .
$$

We also note that if $\pi$ is optimal for $W^{2}(\mu, \lambda)$, then defining for $t \in[0,1]$,

$$
\int \zeta d \rho_{t}=\int \zeta((1-t) x+t y) d \pi \text { and } \int \xi \cdot d j_{t}=\int \xi((1-t) x+t y) \cdot(y-x) d \pi,
$$

the couple $(\rho, j)=\left(\rho_{t} d t, j_{t} d t\right)$ is optimal for 2.18). Using 2.21) in combination with (2.20) and (2.18), we obtain the localized counterpart of (2.18)

$$
\int_{B_{R}} \int_{0}^{1} \frac{1}{\rho}|j|^{2}=\iint_{0}^{1} I(|(1-t) x+t y|<R)|x-y|^{2} d \pi .
$$

We notice that since any admissible $(\rho, j)$ for 2.18$)$ is divergence free (in $(t, x))$, for every $R>0$ it admits internal (and external) traces on $\partial\left(B_{R} \times(0,1)\right)$ (see [19]) i.e. there exists a measure $f$ on $\partial B_{R} \times(0,1)$ such that

$$
\int_{B_{R}} \int_{0}^{1} \partial_{t} \zeta d \rho+\nabla \zeta \cdot d j=\int_{B_{R}} \zeta_{1} d \lambda-\int_{B_{R}} \zeta_{0} d \mu+\int_{\partial B_{R}} \int_{0}^{1} \zeta d f .
$$

Moreover, recalling that $\bar{j}=\int_{0}^{1} d j_{t}$ (see (2.1)), we have

$$
\nabla \cdot \bar{j}=\mu-\lambda
$$

\footnotetext{
iii Indeed, from (2.21) and (2.20), together with $\xi \cdot(y-x)-\frac{1}{2}|\xi|^{2} \leq \frac{1}{2}|x-y|^{2}$ for every $\xi \in \mathbb{R}^{d}$, we have that $\int_{B_{R}} \int_{0}^{1} \frac{1}{\rho}|j|^{2} \leq \int I(|(1-t) x+t y|<R)|x-y|^{2} d \pi$ and the other inequality then follows from 2.18 and another application of 2.20 with $B_{R}$ replaced by $B_{R}^{c}$ to rule out strict inequality.
} 
and also $\bar{j}$ has internaliv (and external) traces on $\partial B_{R}$ which agree for every $R$ such that $|\bar{j}|\left(\partial B_{R}\right)=\mu\left(\partial B_{R}\right)=\lambda\left(\partial B_{R}\right)=0$.

For $R>0, \mu$ and $\lambda$ positive measures on $\partial B_{R}$ with equal mass, we denote (with a slight abuse of notation)

$$
W_{\partial B_{R}}^{2}(\mu, \lambda):=\min _{\pi_{1}=\mu, \pi_{2}=\lambda} \int_{\partial B_{R} \times \partial B_{R}} d_{\partial B_{R}}^{2}(x, y) d \pi,
$$

where $d_{\partial B_{R}}$ denotes the geodesic distance on $\partial B_{R}$. Notice that $W_{\partial B_{R}}^{2}(\mu, \lambda)$ is comparable with the Euclidean Wasserstein distance $W^{2}(\mu, \lambda)$ and that the analog of the Benamou-Brenier formulation (2.18) holds (see [36, Th. 13.8])

$$
W_{\partial B_{R}}^{2}(\mu, \lambda)=\min _{(\rho, j)}\left\{\int_{\partial B_{R}} \int_{0}^{1} \frac{1}{\rho}|j|^{2}: \partial_{t} \rho+\nabla_{\tan } \cdot j=0, \rho_{0}=\mu \text { and } \rho_{1}=\lambda\right\}
$$

The following lemma will be useful in the construction part of the proof of the harmonic approximation result.

Lemma 2.6. Let $f$ and $g$ be two positive measures on $\partial B_{R} \times[0,1]$ such that $\operatorname{Spt} f \subset$ $\partial B_{R} \times\left[0, t_{-}\right]$, Spt $g \subset \partial B_{R} \times\left[t_{+}, 1\right]$ with $\tau:=t_{+}-t_{-}>0$ and $\bar{f}\left(\partial B_{R}\right)=\bar{g}\left(\partial B_{R}\right)$ (where $\bar{f}:=\int_{0}^{1} f$, see (2.1) ). Then, there exists a density-flux pair $(\rho, j)$ on $\partial B_{R} \times$ $[0,1]$ such that

$$
\int_{\partial B_{R}} \int_{0}^{1} \partial_{t} \zeta d \rho+\nabla_{t a n} \zeta \cdot d j=\int_{\partial B_{R}} \int_{0}^{1} \zeta d(g-f)
$$

and

$$
\int_{\partial B_{R}} \int_{0}^{1} \frac{1}{\rho}|j|^{2}=\frac{1}{\tau} W_{\partial B_{R}}^{2}(\bar{f}, \bar{g}) .
$$

Proof. We first connect in $\left(0, t_{-}\right)$, the density $f$ to $\bar{f}$ (at no cost), then in $\left(t_{-}, t_{+}\right)$we connect $\bar{f}$ to $\bar{g}$ (at $\operatorname{cost} \frac{1}{\tau} W_{\partial B_{R}}^{2}(\bar{f}, \bar{g})$ ) and finally connect in $\left(t_{+}, 1\right], \bar{g}$ to $g$ (again at no cost).

More precisely, for $t \in[0, a]$, let

$$
\int \zeta d \rho_{t}=\int_{\partial B_{R}} \int_{0}^{t} \zeta(x) d f(x, s) \quad \text { and } \quad j_{t}:=0
$$

so that

$$
\int_{\partial B_{R}} \int_{0}^{t_{-}} \partial_{t} \zeta d \rho+\nabla_{t a n} \zeta \cdot d j=\int_{\partial B_{R}} \zeta_{t_{-}} d \bar{f}-\int_{\partial B_{R}} \int_{0}^{1} \zeta d f .
$$

Let then $(\widetilde{\rho}, \widetilde{j})$ be a minimizer of the Benamou-Brenier version 2.25 of $W_{\partial B_{R}}(\bar{f}, \bar{g})$ and consider for $t \in\left(t_{-}, t_{+}\right)$the rescaling

$$
\left(\rho_{t}, j_{t}\right):=\left(\widetilde{\rho}_{\frac{t-t_{-}}{\tau}}, \frac{1}{\tau} \widetilde{j}_{\frac{t-t_{-}}{\tau}}\right),
$$

\footnotetext{
${ }^{\text {iv }}$ and of course the internal trace coincides with $\bar{f}=\int_{0}^{1} f$.
} 
so that

$$
\int_{\partial B_{R}} \int_{t_{-}}^{t_{+}} \partial_{t} \zeta d \rho+\nabla_{t a n} \zeta \cdot d j=\int_{\partial B_{R}} \zeta_{t_{+}} d \bar{g}-\int_{\partial B_{R}} \zeta_{t_{-}} d \bar{f}
$$

and

$$
\int_{\partial B_{R}} \int_{t_{-}}^{t_{+}} \frac{1}{\rho}|j|^{2}=\frac{1}{\tau} W_{\partial B_{R}}^{2}(\bar{f}, \bar{g}) .
$$

Finally for $t \in\left(t_{+}, 1\right)$, we let

$$
\int \zeta d \rho_{t}=\int_{\partial B_{R}} \int_{t}^{1} \zeta(x) d g(x, s) \quad \text { and } \quad j_{t}:=0
$$

so that

$$
\int_{\partial B_{R}} \int_{t_{+}}^{1} \partial_{t} \zeta d \rho+\nabla_{t a n} \zeta \cdot d j=\int_{\partial B_{R}} \int_{0}^{1} \zeta d g-\int_{\partial B_{R}} \zeta_{t_{+}} d \bar{g} .
$$

Putting together 2.28, 2.29) and 2.30, we get 2.26).

\subsubsection{A few technical results}

We collect in this section a couple of technical results which will be useful later on.

Lemma 2.7. For every $R>0$ and every measure $\mu$ on $B_{R}$,

$$
W_{B_{R}}(\mu, \kappa) \lesssim W_{B_{R}}(\mu+\kappa d x, 2 \kappa) .
$$

Proof. By the triangle inequality and the monotonicity of $W_{B_{R}}$,

$$
\begin{aligned}
W_{B_{R}}(\mu, \kappa) & \leq W_{B_{R}}\left(\mu, \frac{1}{2}(\mu+\kappa d x)\right)+W_{B_{R}}\left(\frac{1}{2}(\mu+\kappa d x), \kappa\right) \\
& \leq W_{B_{R}}\left(\frac{1}{2} \mu, \frac{1}{2} \kappa\right)+W_{B_{R}}\left(\frac{1}{2}(\mu+\kappa d x), \kappa\right) \\
& =\frac{1}{\sqrt{2}}\left(W_{B_{R}}(\mu, \kappa)+W_{B_{R}}(\mu+\kappa d x, 2 \kappa)\right),
\end{aligned}
$$

which concludes the proof of $(2.31)$.

Lemma 2.8. For every $R>0, \zeta \in C^{\infty}\left(B_{R}\right)$, and $\mu$ supported in $B_{R}$,

$$
\left|\int_{B_{R}} \zeta(d \mu-\kappa d x)\right| \leq\left(\kappa \int_{B_{R}}|\nabla \zeta|^{2}\right)^{\frac{1}{2}} W_{B_{R}}(\mu, \kappa)+\frac{1}{2} \sup _{B_{R}}\left|\nabla^{2} \zeta\right| W_{B_{R}}^{2}(\mu, \kappa) .
$$

Proof. Integrating the Taylor expansion $|\zeta(x)-\zeta(y)-\nabla \zeta(y) \cdot(x-y)| \leq \frac{1}{2} \sup _{B_{R}} \mid \nabla^{2} \zeta \| x-$ $\left.y\right|^{2}$ against an optimal transport plan between $\mu$ and $\kappa d x$ gives (2.32) after an application of Cauchy-Schwarz. 


\subsubsection{An $L^{\infty}$ bound on the displacement}

We now prove an $L^{\infty}$ bound on the transport. This is one of the fundamental ingredients for the proof of Theorem 1.4. It is similar to [26, Lem. 3.1] with the technical difference that we consider here couplings between arbitrary measures.

Lemma 2.9. Let $\pi$ be a coupling between two measures $\mu$ and $\lambda$. Assume that $\operatorname{Spt} \pi$ is monotone and that for some $R>0, \frac{1}{R^{2}} E+\frac{1}{R^{2}} D \ll 1$ where

$$
E:=\frac{1}{\left|B_{6 R}\right|} \int_{\left(B_{6 R} \times \mathbb{R}^{d}\right) \cup\left(\mathbb{R}^{d} \times B_{6 R}\right)}|x-y|^{2} d \pi
$$

and

$$
D:=\frac{1}{\left|B_{6 R}\right|} W_{B_{6 R}}^{2}\left(\mu, \kappa_{\mu}\right)+\frac{R^{2}}{\kappa_{\mu}}\left(\kappa_{\mu}-1\right)^{2}+\frac{1}{\left|B_{6 R}\right|} W_{B_{6 R}}^{2}\left(\lambda, \kappa_{\lambda}\right)+\frac{R^{2}}{\kappa_{\lambda}}\left(\kappa_{\lambda}-1\right)^{2} .
$$

Then, for every $(x, y) \in \operatorname{Spt} \pi \cap\left(\left(B_{5 R} \times \mathbb{R}^{d}\right) \cup\left(\mathbb{R}^{d} \times B_{5 R}\right)\right)$

$$
|x-y| \lesssim R\left(\frac{1}{R^{2}} E+\frac{1}{R^{2}} D\right)^{\frac{1}{d+2}}=: R M .
$$

As a consequence, for $(x, y) \in \operatorname{Spt} \pi$ and $t \in[0,1]$,

$$
(1-t) x+t y \in B_{2 R} \quad \Longrightarrow \quad x \in B_{3 R} \quad \text { or } y \in B_{3 R}
$$

and

$$
x \in B_{R} \quad \text { or } y \in B_{R} \quad \Longrightarrow \quad(1-t) x+t y \in B_{2 R} .
$$

Moreover,

$$
\frac{1}{\left|B_{5 R}\right|} \int_{\left(B_{5 R} \times \mathbb{R}^{d}\right) \cup\left(\mathbb{R}^{d} \times B_{5 R}\right)} d \pi \lesssim 1
$$

Proof. By scaling we may assume that $R=1$. We first prove (2.33). Fix $(x, y) \in$ Spt $\pi \cap\left(\left(B_{5} \times \mathbb{R}^{d}\right) \cup\left(\mathbb{R}^{d} \times B_{5}\right)\right)$. Without loss of generality, we may assume that $(x, y) \in B_{5} \times \mathbb{R}^{d}$. By monotonicity, we then have for every $\left(x^{\prime}, y^{\prime}\right) \in \operatorname{Spt} \pi$ that $\left(y-y^{\prime}\right) \cdot\left(x-x^{\prime}\right) \geq 0$. Writing $y-y^{\prime}=(y-x)+\left(x-x^{\prime}\right)+\left(x^{\prime}-y^{\prime}\right)$ this may be rewritten as

$$
\begin{aligned}
(x-y) \cdot\left(x-x^{\prime}\right) & \leq\left|x-x^{\prime}\right|^{2}+\left(x-x^{\prime}\right) \cdot\left(x^{\prime}-y^{\prime}\right) \\
& \leq \frac{3}{2}\left|x-x^{\prime}\right|^{2}+\frac{1}{2}\left|x^{\prime}-y^{\prime}\right|^{2} .
\end{aligned}
$$

Integrating this inequality against the measure $\eta\left(x^{\prime}\right) d \pi\left(x^{\prime}, y^{\prime}\right)$ for some smooth function $\eta \in[0,1]$ supported in $B_{6}$ we get

$$
\begin{aligned}
&(x-y) \cdot \int\left(x-x^{\prime}\right) \eta\left(x^{\prime}\right) d \mu\left(x^{\prime}\right) \lesssim \int\left|x-x^{\prime}\right|^{2} \eta\left(x^{\prime}\right) d \mu\left(x^{\prime}\right)+\int_{B_{6} \times \mathbb{R}^{d}}\left|x^{\prime}-y^{\prime}\right|^{2} d \pi \\
& \lesssim \int\left|x-x^{\prime}\right|^{2} \eta\left(x^{\prime}\right) d \mu\left(x^{\prime}\right)+E
\end{aligned}
$$


We now fix an arbitrary unit vector $e$ and a radius $0<r \ll 1$ and assume that $\eta$ is supported in $B_{r}(x-r e)$ (which is contained in $B_{6}$ since $\left.x \in B_{5}\right)$ with $r \sup |\nabla \eta|+$ $r^{2}\left|\nabla^{2} \eta\right| \lesssim 1$. We want to replace the measure $\mu$ by the Lebesgue measure and for this use (2.32) and $\kappa_{\mu} \sim 1$ to get

$$
\left|\int\left(x-x^{\prime}\right) \eta\left(x^{\prime}\right) d \mu\left(x^{\prime}\right)-\kappa_{\mu} \int\left(x-x^{\prime}\right) \eta\left(x^{\prime}\right) d x^{\prime}\right| \lesssim\left(r^{d} D\right)^{\frac{1}{2}}+\frac{1}{r} D
$$

and

$$
\left|\int\right| x-\left.x^{\prime}\right|^{2} \eta\left(x^{\prime}\right) d \mu\left(x^{\prime}\right)-\kappa_{\mu} \int\left|x-x^{\prime}\right|^{2} \eta\left(x^{\prime}\right) d x^{\prime} \mid \lesssim\left(r^{d+2} D\right)^{\frac{1}{2}}+D .
$$

Appropriately normalizing $\eta$ and choosing it to be radially symmetric around $x-r e$ we have

$$
\int\left(x-x^{\prime}\right) \eta\left(x^{\prime}\right) d x^{\prime}=r^{d+1} e \quad \text { and } \quad \int\left|x-x^{\prime}\right|^{2} \eta\left(x^{\prime}\right) d x^{\prime} \lesssim r^{d+2} .
$$

Using again that $\kappa_{\mu} \sim 1,2.38$ and $2.39,2.37$ becomes

$$
r^{d+1}(x-y) \cdot e \lesssim\left(\left(r^{d} D\right)^{\frac{1}{2}}+\frac{1}{r} D\right)|x-y|+r^{d+2}+\left(r^{d+2} D\right)^{\frac{1}{2}}+D+E .
$$

Since $e$ is arbitrary, using Young's inequality i.e. $\left(r^{d} D\right)^{\frac{1}{2}} \lesssim \varepsilon r^{d+1}+\frac{1}{r \varepsilon} D$, this yields

$$
r^{d+1}|x-y| \lesssim \frac{1}{r} D|x-y|+r^{d+2}+D+E .
$$

Choosing $r$ to be a large multiple of $(E+D)^{\frac{1}{d+2}}$, we conclude the proof of 2.33 .

To show (2.36) we estimate

$$
\frac{1}{\left|B_{5}\right|} \int_{\left(B_{5} \times \mathbb{R}^{d}\right) \cup\left(\mathbb{R}^{d} \times B_{5}\right)} d \pi \leq \frac{1}{\left|B_{5}\right|} \int_{B_{6} \times \mathbb{R}^{d}} d \pi+\frac{1}{\left|B_{5}\right|} \int_{\mathbb{R}^{d} \times B_{6}} d \pi=\frac{\mu\left(B_{6}\right)+\lambda\left(B_{6}\right)}{\left|B_{5}\right|} \lesssim \kappa_{\mu}+\kappa_{\lambda}
$$

so that 2.36) follows from $\kappa_{\mu}+\kappa_{\lambda} \sim 1$. Since 2.35) is a direct consequence of (2.33), we are left with the proof of 2.34 . Since $E+D \ll 1$, there exists $\left(x^{\prime}, y^{\prime}\right) \in \operatorname{Spt} \pi$ with $\left|x^{\prime}\right|+\left|y^{\prime}\right| \ll 1$. By monotonicity of Spt $\pi$, we then have

$$
\begin{aligned}
4+o(1) & \geq\left|(1-t) x+t y-(1-t) x^{\prime}-t y^{\prime}\right|^{2} \\
& =(1-t)^{2}\left|x-x^{\prime}\right|^{2}+2 t(1-t)\left(x-x^{\prime}\right) \cdot\left(y-y^{\prime}\right)+t^{2}\left|y-y^{\prime}\right|^{2} \\
& \geq(1-t)^{2}\left|x-x^{\prime}\right|^{2}+t^{2}\left|y-y^{\prime}\right|^{2} \\
& \geq \frac{1}{2} \min \left(\left|x-x^{\prime}\right|^{2},\left|y-y^{\prime}\right|^{2}\right) \geq \frac{1}{2} \min \left(|x|^{2},|y|^{2}\right)-o(1),
\end{aligned}
$$

which concludes the proof. 


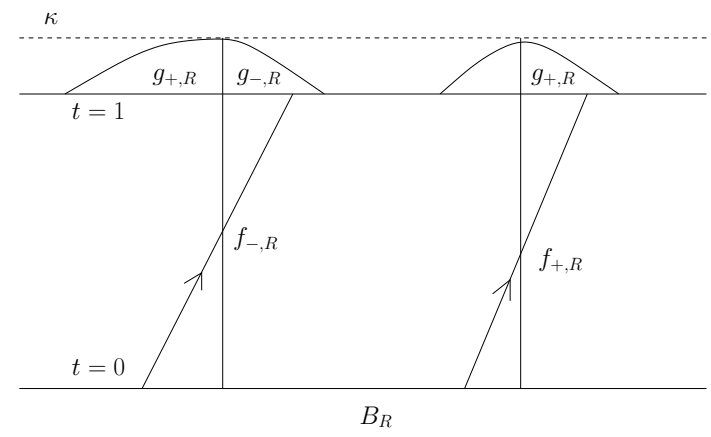

Figure 2: The definition of $g_{ \pm, R}$.

\subsubsection{A localization result}

Our next lemma shows that the quantity $W_{B_{5}}^{2}(\mu, \kappa)+\frac{1}{\kappa}(\kappa-1)^{2}$, which captures the (squared) distance of a measure $\mu$ to the Lebesgue measure, is well behaved under restricting $\mu$ to balls $B_{R}$ with $R \sim 1$. However, this seems true only on average in $R$. Since in the application to the matching problem we will need the lemma for cubes instead of balls, we will state it in a general form.

Lemma 2.10. We have for any positive measure $\mu$

$$
\int_{3}^{4}\left(W_{B_{R}}^{2}\left(\mu, \kappa_{R}\right)+\frac{1}{\kappa_{R}}\left(\kappa_{R}-1\right)^{2}\right) d R \lesssim D
$$

provided

$$
D:=W_{O}^{2}(\mu, \kappa)+\frac{1}{\kappa}(\kappa-1)^{2} \ll 1,
$$

for some set $O \supset B_{5}$.

Before starting the proof, let us point out that the proofs in Section 3.1 use similar ideas and techniques in a slightly more complicated setup. Hence, inside the proof we use similar notation.

Proof. Let $\pi$ denote the optimal transference plan for $W_{O}^{2}(\mu, \kappa)$. It is convenient to introduce the corresponding (non-normalized) measure $\mathbb{P} \geq 0$ on straight trajectories $[0,1] \ni t \mapsto X(t)$ : It is the push-forward of $\pi$ under $X(t)=t y+(1-t) x$. For given $R \in(0,5)$ consider the set of exiting and entering trajectories, respectively (see Figure 2),

$$
\begin{aligned}
& \Omega_{+, R}:=\{X|| X(0)|<R,| X(1) \mid \geq R\}, \\
& \Omega_{-, R}:=\{X|| X(0)|\geq R,| X(1) \mid<R\} .
\end{aligned}
$$

We define the measures $0 \leq g_{+, R} \leq \kappa$ on $B_{R}^{c}$ and $0 \leq g_{-, R} \leq \kappa$ on $B_{R}$ which record where exiting and entering trajectories end up:

$$
\int \zeta d g_{ \pm, R}:=\int_{\Omega_{ \pm, R}} \zeta(X(1)) d \mathbb{P} .
$$


We claim that these measures are, on average in $R$, concentrated close to $\partial B_{R}$ :

$$
\int_{3}^{4}\left(\int(|x|-R) d g_{+, R}+\int(R-|x|) d g_{-, R}\right) d R \leq W_{O}^{2}(\mu, \kappa) .
$$

Indeed, by definition 2.42 of $g_{ \pm, R}$, we have

$$
\begin{aligned}
& \int(|x|-R) d g_{+, R}+\int(R-|x|) d g_{-, R} \\
& =\int_{|X(0)|<R \leq|X(1)|}(|X(1)|-R) d \mathbb{P}+\int_{|X(1)|<R \leq|X(0)|}(R-|X(1)|) d \mathbb{P} \\
& \leq \int_{\min \{|X(0)|,|X(1)|\}<R \leq \max \{|X(0)|,|X(1)|\}}|| X(1)|-| X(0)|| d \mathbb{P},
\end{aligned}
$$

so that

$$
\begin{aligned}
& \int_{3}^{4}\left(\int(|x|-R) d g_{+, R}+\int(R-|x|) d g_{-, R}\right) d R \\
& \leq \int|\max \{|X(0)|,|X(1)|\}-\min \{|X(0)|,|X(1)|\}||X(1)|-|X(0)| \mid d \mathbb{P} \\
& \leq \int|X(1)-X(0)|^{2} d \mathbb{P}=W_{O}^{2}(\mu, \kappa) .
\end{aligned}
$$

Momentarily fixing $R$ and setting

$$
\kappa_{+, R}:=\frac{1}{\left|B_{R}\right|} g_{+, R}\left(\mathbb{R}^{d}\right), \quad \kappa_{-, R}:=\frac{1}{\left|B_{R}\right|} g_{-, R}\left(\mathbb{R}^{d}\right),
$$

we claim that for every $R \in(3,4)$ with $\mu\left(\partial B_{R}\right)=0$,

$$
W_{B_{R}}^{2}\left(\mu, \kappa_{R}\right) \lesssim W_{O}^{2}(\mu, \kappa)+\int(|x|-R) d g_{+, R}+\int(R-|x|) d g_{-, R},
$$

where

$$
\kappa_{R}:=\kappa-\kappa_{-, R}+\kappa_{+, R} .
$$

We first notice that thanks to the $L^{\infty}$ bound 2.33, and the definitions 2.41), 2.42 and 2.44), for any $R \in(3,4)$,

$$
\kappa_{ \pm, R}=\frac{1}{\left|B_{R}\right|} \mathbb{P}\left(\Omega_{ \pm, R}\right) \stackrel{\sqrt[2.33]{\lesssim}}{\lesssim} \mathbb{P}\left(|| X(1)|-R| \lesssim D^{\frac{1}{d+2}}\right) \stackrel{2.40}{\ll} \kappa .
$$

${ }^{v}$ Notice that by 2.41) (while the test function $I(|x|<R$ ) is not continuous, a simple approximation argument shows that this is actually not an issue),

$$
\mu\left(B_{R}\right)=\mathbb{P}(|X(0)|<R)=\mathbb{P}(|X(1)|<R)-\mathbb{P}\left(\Omega_{-, R}\right)+\mathbb{P}\left(\Omega_{+, R}\right)=\left|B_{R}\right|\left(\kappa-\kappa_{-, R}+\kappa_{+, R}\right) .
$$


We now turn to $(2.45)$ and to this purpose introduce the measure $f_{+, R} \geq 0$ on $\partial B_{R} \times[0,1]$ that keeps track of where and when trajectories exit (see Figure 2)

$$
\int \zeta d f_{+, R}:=\int_{\Omega_{+, R}} \zeta\left(X\left(t_{+}\right), t_{+}\right) d \mathbb{P},
$$

here $t_{+}$denotes the unique $t_{+} \in(0,1]$ such that $\left|X\left(t_{+}\right)\right|=R$. We also introduce the measures $\hat{g}_{ \pm, R} \geq 0$ and $\bar{f}_{+, R} \geq 0$ on $\partial B_{R}$ by projection (note that $\hat{g}_{ \pm, R}$ has a density)

$$
\int \zeta d \hat{g}_{ \pm, R}:=\int \zeta\left(R \frac{x}{|x|}\right) d g_{ \pm, R}(x), \quad \int \zeta d \bar{f}_{+, R}:=\int \zeta(x) d f_{+, R}(x, t) .
$$

We now use the triangle inequality for $W_{B_{R}}$ and to split the estimate 2.45 into

$$
\begin{aligned}
W_{B_{R}}^{2}\left(\mu, \kappa_{R}\right) \lesssim & W_{\bar{B}_{R}}^{2}\left(\mu, \kappa d x-g_{-, R}+\bar{f}_{+, R}\right) \\
& +W_{\bar{B}_{R}}^{2}\left(\kappa d x-g_{-, R}+\bar{f}_{+, R},\left(\kappa-\kappa_{-, R}\right) d x+\hat{g}_{+, R}\right) \\
& +W_{\bar{B}_{R}}^{2}\left(\left(\kappa-\kappa_{-, R}\right) d x+\hat{g}_{+, R}, \kappa_{R}\right),
\end{aligned}
$$

and then the sub-additivity of $W_{\bar{B}_{R}}^{2}$ to split the middle term further into

$$
\begin{aligned}
W_{\bar{B}_{R}}^{2}\left(\kappa d x-g_{-, R}+\bar{f}_{+, R},\right. & \left.\left(\kappa-\kappa_{-, R}\right) d x+\hat{g}_{+, R}\right) \\
\leq & W_{B_{R}}^{2}\left(\kappa d x-g_{-, R}, \kappa-\kappa_{-, R}\right)+W_{\partial B_{R}}^{2}\left(\bar{f}_{+, R}, \hat{g}_{+, R}\right) .
\end{aligned}
$$

We start with the two easier terms. We claim that the first right-hand side term in 2.49 is estimated as follows

$$
W_{\bar{B}_{R}}^{2}\left(\mu, \kappa d x-g_{-, R}+\bar{f}_{+, R}\right) \leq W_{O}^{2}(\mu, \kappa) .
$$

Indeed, an admissible transference plan $\pi_{R}$ is given by restricting the plan $\pi$

$$
\int \zeta d \pi_{R}=\int_{|X(0)|<R,|X(1)|<R} \zeta(X(0), X(1)) d \mathbb{P}+\int_{\Omega_{+, R}} \zeta\left(X(0), X\left(t_{+}\right)\right) d \mathbb{P} .
$$

Likewise, the second right-hand side term of $(2.50)$ is estimated in the same way:

$$
W_{\partial B_{R}}^{2}\left(\bar{f}_{+, R}, \hat{g}_{+, R}\right) \leq W_{O}^{2}(\mu, \kappa) .
$$

Again, an admissible transference plan $\pi_{+, R}$ is given by

$$
\int \zeta d \pi_{+, R}=\int_{\Omega_{+, R}} \zeta\left(X\left(t_{+}\right), R \frac{X(1)}{|X(1)|}\right) d \mathbb{P} .
$$

We now turn to the remaining two more subtle terms. For the last right-hand side term in (2.49) we claim that

$$
W_{\bar{B}_{R}}^{2}\left(\left(\kappa-\kappa_{-, R}\right) d x+\hat{g}_{+, R}, \kappa_{R}\right) \lesssim \int(|x|-R) d g_{+, R} .
$$


Indeed, recalling the definition (2.46) of $\kappa_{R}$, an admissible density-flux pair $(\rho, j)$ for the Benamou-Brenier formulation (2.18) is given by

$$
\rho_{t}:=\left(\kappa-\kappa_{-, R}+t \kappa_{+, R}\right) d x+(1-t) \hat{g}_{+, R}, \quad j_{t}:=\left\{\begin{array}{cl}
\nabla \varphi & \text { in } B_{R} \\
0 & \text { else }
\end{array}\right\},
$$

where $\varphi$ is a solution of

$$
\Delta \varphi=-\kappa_{+, R} \text { in } B_{R} \quad \text { and } \quad \nu \cdot \nabla \varphi=-\hat{g}_{+, R} \text { on } \partial B_{R},
$$

so that we have

$$
W_{\bar{B}_{R}}^{2}\left(\left(\kappa-\kappa_{-, R}\right) d x+\hat{g}_{+, R}, \kappa_{R}\right) \leq \frac{1}{\kappa-\kappa_{-, R}} \int_{B_{R}}|\nabla \varphi|^{2} .
$$

Combining this with the energy estimate 2.11 (applied to $f=\hat{g}_{+, R}$ and $g=0$ ), (2.33) to be able to apply (2.13) and 2.47), this concludes the proof of (2.51).

Likewise, the first right-hand side term in 2.50 is estimated as

$$
W_{B_{R}}^{2}\left(\kappa d x-g_{-, R}, \kappa-\kappa_{-, R}\right) \lesssim \int(R-|x|) d g_{-, R} .
$$

Indeed, by (2.31) it is a consequence of

$$
W_{B_{R}}^{2}\left(\left(2 \kappa-\kappa_{-, R}\right) d x-g_{-, R}, 2\left(\kappa-\kappa_{-, R}\right)\right) \lesssim \int(R-|x|) d g_{-, R} .
$$

In turn, this follows arguing as in (2.51) and using as admissible density-flux pair $(\rho, j)$

$$
\rho_{t}:=2 \kappa-\kappa_{-, R}-t \kappa_{-, R}-(1-t) g_{-, R}, \quad j_{t}:=\left\{\begin{array}{cl}
\nabla \varphi & \text { in } B_{R} \\
0 & \text { else }
\end{array}\right\}
$$

where $\varphi$ is a solution of

$$
\Delta \varphi=\kappa_{-, R}-g_{-, R} \text { in } B_{R} \quad \text { and } \quad \nu \cdot \nabla \varphi=0 \text { on } \partial B_{R} .
$$

This concludes the proof of 2.45).

Finally, by the definition 2.46) of $\kappa_{R}$ and (2.47),

$$
\frac{1}{\kappa_{R}}\left(1-\kappa_{R}\right)^{2} \lesssim \frac{1}{\kappa}(1-\kappa)^{2}+\kappa_{-, R}^{2}+\kappa_{+, R}^{2}
$$

Combining this with $\kappa_{ \pm, R}=\frac{1}{\left|B_{R}\right|} g_{ \pm, R}\left(\mathbb{R}^{d}\right)$ (cf. 2.44) and 2.13), we obtain

$$
\frac{1}{\kappa_{R}}\left(1-\kappa_{R}\right)^{2} \lesssim \frac{1}{\kappa}(1-\kappa)^{2}+\int(|x|-R) d g_{+, R}+\int(R-|x|) d g_{-, R},
$$

which together with 2.45 and 2.43 concludes the proof of the lemma. 
Remark 2.11. Let us point out that with a very similar proof, it may be shown that if $\pi$ is the optimal coupling between a measure $\mu$ and the Lebesgue measure for which $E+D \ll 1$ where

$$
E:=\frac{1}{\left|B_{5}\right|} \int_{\left(B_{5} \times \mathbb{R}^{d}\right) \cup\left(\mathbb{R}^{d} \times B_{5}\right)}|x-y|^{2} d \pi \quad \text { and } \quad D:=\frac{1}{\left|B_{5}\right|} W_{B_{5}}^{2}(\mu, \kappa),
$$

then

$$
\int_{3}^{4}\left(W_{B_{R}}^{2}\left(\mu, \kappa_{R}\right)+\frac{1}{\kappa_{R}}\left(\kappa_{R}-1\right)^{2}\right) d R \lesssim E+D .
$$

In particular, this means that when the target measure is the Lebesgue measure, up to choosing a good radius, we have a good control on $\left.\frac{1}{\kappa}(\kappa-1)^{2}\right)$.

\section{The harmonic approximation result}

In this section, which is the core of the paper, we prove Theorem 1.4. As already explained, by scaling we will prove it on the unit scale. For $\pi$ an optimal coupling between two measures $\mu$ and $\lambda$, we recall that we set

$$
\begin{aligned}
& E=\frac{1}{\left|B_{6}\right|} \int_{\left(B_{6} \times \mathbb{R}^{d}\right) \cup\left(\mathbb{R}^{d} \times B_{6}\right)}|x-y|^{2} d \pi, \\
& D=\frac{1}{\left|B_{6}\right|} W_{B_{6}}^{2}\left(\mu, \kappa_{\mu}\right)+\frac{1}{\kappa_{\mu}}\left(\kappa_{\mu}-1\right)^{2}+\frac{1}{\left|B_{6}\right|} W_{B_{6}}^{2}\left(\lambda, \kappa_{\lambda}\right)+\frac{1}{\kappa_{\lambda}}\left(\kappa_{\lambda}-1\right)^{2}
\end{aligned}
$$

and work under the hypothesis that $E+D \ll 1$. As explained in the introduction, the strategy of the proof is to argue at the Eulerian level and first choose a good radius $R \in(3,4)$ for which the solution $\Phi$ (respectively $\phi$ ) of the Poisson equation in $B_{R}$ with Neumann boundary condition given by the Eulerian flux (respectively a regularized version of ) $\bar{j}$ on $\partial B_{R}$ is 'well-behaved'. This is done in Section 3.1. Then, we prove in Section 3.2.1 an approximate orthogonality result, which combined with the construction of a competitor with controlled energy in Section 3.2 .2 and the minimality of the density-flux pair $(\rho, j)$ yields Theorem 1.4 .

\subsection{Choice of a good radius}

In this section we define the main densities and fluxes which will appear in the proof of Theorem 1.4 and prove that we may choose a good radius for which they are well-behaved.

\subsubsection{Unregularized fluxes}

Using the Gluing Lemma (see [35, Lem. 7.6]) and disintegration, we couple the optimal transport between $\mu$ and $\lambda$ on the time interval $t \in(0,1)$ to the optimal transport between $\kappa_{\mu}$ and $\mu$ (restricted to $\left.B_{6} \times B_{6}\right)$ in the interval $(-1,0)$ and to the optimal transport between $\lambda$ and $\kappa_{\lambda}$ (restricted to $\left.B_{6} \times B_{6}\right)$ in the interval $(1,2)$. In view of Lemma 2.9 , there exists a (not normalized) measure $\mathbb{P}$ on continuous 
trajectories $[-1,2] \ni t \mapsto X(t) \in \mathbb{R}^{d}$ that couples $\kappa_{\mu} d x, \mu, \lambda$, and $\kappa_{\lambda} d x$ in the sense that for any $\zeta \in C_{c}^{0}\left(B_{5}\right)$ (see Figure 1)

$$
\begin{aligned}
\int \zeta(X(-1)) d \mathbb{P} & =\int \zeta \kappa_{\mu} d x, \\
\int \zeta(X(0)) d \mathbb{P} & =\int \zeta d \mu, \\
\int \zeta(X(1)) d \mathbb{P} & =\int \zeta d \lambda, \\
\int \zeta(X(2)) d \mathbb{P} & =\int \zeta \kappa_{\lambda} d x .
\end{aligned}
$$

Moreover, the trajectories are piecewise affine on $(-1,0) \cup(0,1) \cup(1,2)$ and optimal in the sense that

$$
\begin{array}{lll}
\int|X(0)-X(-1)|^{2} d \mathbb{P} & \leq W_{B_{6}}^{2}\left(\mu, \kappa_{\mu}\right) & \leq\left|B_{6}\right| D \\
\int|X(1)-X(0)|^{2} d \mathbb{P} & & \leq\left|B_{6}\right| E \\
\int|X(2)-X(1)|^{2} d \mathbb{P} & \leq W_{B_{6}}^{2}\left(\lambda, \kappa_{\lambda}\right) & \leq\left|B_{6}\right| D .
\end{array}
$$

Finally, by (2.21) and (3.1), the density/flux pair $\left(\rho=\rho_{t} d t, j=j_{t} d t\right)$ can be recovered from $\mathbb{P}$ on $B_{5} \times(0,1)$ in the sense that for $t \in[0,1], \zeta \in C_{c}^{0}\left(B_{5}\right)$, and $\xi \in C_{c}^{0}\left(B_{5}\right)^{d}$

$$
\begin{aligned}
\int \zeta(X(t)) d \mathbb{P} & =\int \zeta d \rho_{t}, \\
\int \xi(X(t)) \cdot \dot{X}(t) d \mathbb{P} & =\int \xi \cdot d j_{t} .
\end{aligned}
$$

We then recall the definitions of the time integrated fluxes (see 2.1)

$$
\bar{\rho}=\int_{0}^{1} \rho_{t} d t \quad \text { and } \quad \bar{j}=\int_{0}^{1} j_{t} d t
$$

Let us notice that using $(2.34)$ (in combination with $E+D \ll 1$ ) on the one hand and 3.2 together with 3.3 on the other hand, we have

$$
\int_{B_{2}} d \bar{\rho} \lesssim 1 \quad \text { and } \quad \int_{B_{5}} \int_{0}^{1} \frac{1}{\rho}|j|^{2} \leq E .
$$

In the upcoming Lemma 3.1, we derive that for any radius $R$, the (inner) flux boundary data $f_{R}$ of $j$ on $\partial B_{R} \times[0,1]$ admit a representation in terms of $\mathbb{P}$. Indeed, consider the set $\Omega_{+, R}$ of trajectories that exit $B_{R}$ and the set $\Omega_{-, R}$ of those that enter:

$$
\begin{aligned}
& \Omega_{+, R}:=\{X|| X(1) \mid \geq R \text { and }|X(t)|<R \text { for some } t \in[0,1]\}, \\
& \Omega_{-, R}:=\{X|| X(0) \mid \geq R \text { and }|X(t)|<R \text { for some } t \in[0,1]\} .
\end{aligned}
$$

We notice that these sets are not necessarily disjoint. We also define the set of trajectories $\Omega_{R}$ which enter, exit or are entirely contained in $B_{R}$ :

$$
\Omega_{R}:=\{X|| X(t) \mid<R \text { for some } t \in[0,1]\}
$$


and point out that by the $L^{\infty}$ bound (2.33), if $E+D \ll 1$ then for $R \leq 5, X \in \Omega_{R}$ and $(s, t) \in[-1,2]$,

$$
|X(t)-X(s)| \lesssim M
$$

where we recall that $M=(E+D)^{\frac{1}{d+2}}$. We associate exiting and entering times to the trajectories that spend time in $B_{R}$ :

$$
\left.\begin{array}{ll}
t_{+}:=\sup \{t \in[0,1]|| X(t) \mid<R\} & \in(0,1] \\
t_{-}:=\inf \{t \in[0,1]|| X(t) \mid<R\} & \in[0,1)
\end{array}\right\} \quad \text { for } X \in \Omega_{R}
$$

note that $t_{+}=1$ if the trajectory ends in $\bar{B}_{R}$, and $t_{-}=0$ if the trajectory starts in $\bar{B}_{R}$. We introduce the non-negative measures $f_{ \pm, R}$ on $\partial B_{R} \times[0,1]$ that keep track of where in space-time trajectories exit and enter:

$$
\int \zeta d f_{ \pm, R}=\int_{\Omega_{ \pm, R}} \zeta\left(X\left(t_{ \pm}\right), t_{ \pm}\right) d \mathbb{P}
$$

We are now in the position to introduce signed measure on $\partial B_{R} \times[0,1]$ :

$$
f_{R}:=f_{+, R}-f_{-, R}
$$

Lemma 3.1. For every $R \in(0,5)$, the measure $f_{R}$ coincides with the inner trace of $j$ on $\partial B_{R} \times(0,1)$ in the sense of 2.23$)$ i.e. for every $\zeta \in C_{c}^{1}\left(\mathbb{R}^{d} \times[0,1]\right)$

$$
\int_{B_{R}} \int_{0}^{1} \partial_{t} \zeta d \rho+\nabla \zeta \cdot d j=\int_{B_{R}}\left(\zeta_{1} d \lambda-\zeta_{0} d \mu\right)+\int_{\partial B_{R}} \int_{0}^{1} \zeta d f_{R} .
$$

As a consequence, for every pair $(\widetilde{\rho}, \widetilde{j})$ satisfying

$$
\iint_{0}^{1} \partial_{t} \zeta d \widetilde{\rho}+\nabla \zeta \cdot \widetilde{d j}=\int_{B_{R}}\left(\zeta_{1} d \lambda-\zeta_{0} d \mu\right)+\int_{\partial B_{R}} \int_{0}^{1} \zeta d f_{R}
$$

there holds,

$$
\int_{B_{R}} \int_{0}^{1} \frac{1}{\rho}|j|^{2} \leq \iint_{0}^{1} \frac{1}{\widetilde{\rho}}|\widetilde{j}|^{2}
$$

Proof. We first prove 3.12 . For $\zeta \in C_{c}^{1}\left(\mathbb{R}^{d} \times[0,1]\right)$, by definition 3.3$)$ of $(\rho, j)$ vi

$$
\begin{aligned}
& \int_{B_{R}} \int_{0}^{1} \partial_{t} \zeta d \rho+\nabla \zeta \cdot d j \\
& =\iint_{0}^{1}\left[\partial_{t} \zeta(X(t), t)+\nabla \zeta(X(t), t) \cdot \dot{X}(t)\right] I(|X(t)|<R) d t d \mathbb{P} \\
& =\int_{\Omega_{R}}\left(\zeta\left(X\left(t_{+}\right), t_{+}\right)-\zeta\left(X\left(t_{-}\right), t_{-}\right)\right) d \mathbb{P}
\end{aligned}
$$

\footnotetext{
${ }^{\mathrm{vi}}$ as in the proof of Lemma 2.10, it can be seen that we may use the (discontinuous) test functions $I(|x|<R) \partial_{t} \zeta$ and $I(|x|<R) \nabla \zeta$.
} 


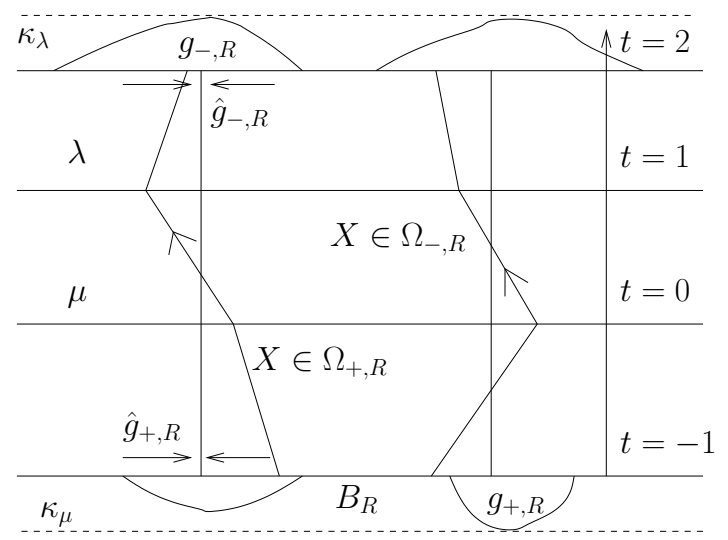

Figure 3: The regularized fluxes $\hat{g}_{ \pm}$.

Now by (3.6), $\Omega_{+, R}=\Omega_{R} \cap\{X|| X(1) \mid \geq R\}$ and thus,

$$
\begin{aligned}
\int_{\Omega_{R}} \zeta\left(X\left(t_{+}\right), t_{+}\right) d \mathbb{P}= & \int_{\Omega_{R}} \zeta(X(1), 1) I(|X(1)|<R) d \mathbb{P} \\
& +\int_{\Omega_{R}} \zeta\left(X\left(t_{+}\right), t_{+}\right) I(|X(1)| \geq R) d \mathbb{P} \\
& \stackrel{3.1] \&(3.10)}{=} \int_{B_{R}} \zeta_{1} d \lambda+\int_{\partial B_{R}} \int_{0}^{1} \zeta d f_{+, R} .
\end{aligned}
$$

Analogously,

$$
\int_{\Omega_{R}} \zeta\left(X\left(t_{-}\right), t_{-}\right) d \mathbb{P}=\int_{B_{R}} \zeta_{0} d \mu+\int_{\partial B_{R}} \int_{0}^{1} \zeta d f_{-, R}
$$

which concludes the proof of $(3.12)$.

Let now $(\widetilde{\rho}, \widetilde{j})$ be a pair satisfying $(3.13)$. The pair $(\check{\rho}, \check{j}):=(\widetilde{\rho}, \widetilde{j})+(\rho, j)\left\llcorner B_{R}^{c}\right.$ is then a competitor for 2.18 so that by subadditivity of $\int \frac{1}{\rho}|j|^{2}$ (which follows from (2.20),

$$
\int \frac{1}{\rho}|j|^{2} \leq \int \frac{1}{\check{\rho}}|\check{j}|^{2} \leq \int \frac{1}{\widetilde{\rho}}|\widetilde{j}|^{2}+\int_{B_{R}^{c}} \int_{0}^{1} \frac{1}{\rho}|j|^{2}
$$

from which (3.14) follows.

\subsubsection{Regularized fluxes and good radius for the approximate or- thogonality}

We now introduce the regularized boundary flux (see Figure 3 )

$$
\hat{g}_{R}:=\hat{g}_{+, R}-\hat{g}_{-, R}
$$


where $\hat{g}_{+, R} \geq 0$ and $\hat{g}_{-, R} \geq 0$ on $\partial B_{R}$ are defined through the projection onto $\partial B_{R}$ of where these trajectories started and ended, respectively,

$$
\int \zeta d \hat{g}_{+, R}:=\int_{\Omega_{+, R}} \zeta\left(R \frac{X(-1)}{|X(-1)|}\right) d \mathbb{P}, \quad \int \zeta d \hat{g}_{-, R}:=\int_{\Omega_{-, R}} \zeta\left(R \frac{X(2)}{|X(2)|}\right) d \mathbb{P} .
$$

From the definition it follows that these measures have densities. We will show that these densities are square integrable and $\hat{g}_{ \pm, R}$ are close to the following non-negative measures on $\partial B_{R}$ :

$$
\int \zeta d \bar{f}_{ \pm, R}:=\int_{\Omega_{ \pm, R}} \zeta\left(X\left(t_{ \pm}\right)\right) d \mathbb{P}
$$

For later reference, we also introduce the notation

$$
\bar{f}_{R}:=\bar{f}_{+, R}-\bar{f}_{-, R} .
$$

Lemma 3.2 (Approximation). With $M=(E+D)^{\frac{1}{d+2}}$ we have

$$
\begin{array}{r}
\int_{3}^{4} \int_{\partial B_{R}} \hat{g}_{ \pm, R}^{2} d R \lesssim E+D, \\
\int_{3}^{4} W_{\partial B_{R}}^{2}\left(\hat{g}_{ \pm, R}, \bar{f}_{ \pm, R}\right) d R \lesssim M(E+D) .
\end{array}
$$

Proof. By symmetry, we can focus on the case with the +-sign (exiting trajectories). We consider the measure $0 \leq g_{+, R} \leq \kappa_{\mu}$ on $\mathbb{R}^{d}$ where trajectories in $\Omega_{+, R}$ initially started (so that in particular $\hat{g}_{+, R}=\Pi_{R} \# g_{+, R}$, where $\Pi_{R}$ is the projection on $\partial B_{R}$ )

$$
\int \zeta d g_{+, R}=\int_{\Omega_{+, R}} \zeta(X(-1)) d \mathbb{P}
$$

and claim that it is concentrated near $\partial B_{R}$ in the sense that

$$
\int_{3}^{4} \int|R-| x|| d g_{+, R} d R \lesssim E+D .
$$

Then, 3.19 would follow from 2.13.

By definition (3.21) of $g_{+, R}$ and definition 3.9 of $t_{+}$on $\Omega_{+, R}$ we have

$$
\int|R-| x|| d g_{+, R}=\int_{\Omega_{+}, R}|| X\left(t_{+}\right)|-| X(-1)|| d \mathbb{P}
$$

and thus by the triangle inequality and the straightness of trajectories on $(0,1)$

$$
\int|R-| x|| d g_{+, R} \leq \int_{\Omega_{+, R}}(|X(1)-X(0)|+|X(0)-X(-1)|) d \mathbb{P} .
$$

Furthermore, since by definition (3.6), for $X \in \Omega_{+, R}$ we have $|X(1)| \geq R \geq \min _{[0,1]}|X|$ and thus

$$
\int_{0}^{\infty} I\left(X \in \Omega_{+, R}\right) d R=|X(1)|-\min _{[0,1]}|X| \leq|X(1)-X(0)|
$$


Combining (3.23) with (3.24) we obtain

$$
\begin{aligned}
& \int_{3}^{4} \int|R-| x|| d g_{+, R} d R \\
& \leq \int|X(1)-X(0)|(|X(1)-X(0)|+|X(0)-X(-1)|) d \mathbb{P} .
\end{aligned}
$$

By Cauchy-Schwarz and $(3.2)$ this yields as desired $\int_{3}^{4} \int(R-|x|) d g_{+, R} d R \leq E+$ $(E D)^{\frac{1}{2}}$.

We now turn to $(3.20)$. It follows from the definitions $(3.16)$ and $(3.17)$ that

$$
\int \zeta(x, y) d \pi_{R}(x, y)=\int_{\Omega_{+, R}} \zeta\left(X\left(t_{+}\right), R \frac{X(-1)}{|X(-1)|}\right) d \mathbb{P}
$$

defines a coupling $\pi_{R}$ between $\bar{f}_{+, R}$ and $\hat{g}_{+, R}$ so that in particular

$$
W_{\partial B_{R}}^{2}\left(\bar{f}_{+, R}, \hat{g}_{+, R}\right) \lesssim W^{2}\left(\bar{f}_{+, R}, \hat{g}_{+, R}\right) \leq \int_{\Omega_{+, R}}\left|X\left(t_{+}\right)-R \frac{X(-1)}{|X(-1)|}\right|^{2} d \mathbb{P} .
$$

Given $X \in \Omega_{+, R}$ note that since $\left|X\left(t_{+}\right)\right|=R$ we have (recall $R \in(3,4)$ )

$$
\left|X\left(t_{+}\right)-R \frac{X(-1)}{|X(-1)|}\right|=R\left|\frac{X\left(t_{+}\right)}{\left|X\left(t_{+}\right)\right|}-\frac{X(-1)}{|X(-1)|}\right| \leq 4\left|\frac{X\left(t_{+}\right)}{\left|X\left(t_{+}\right)\right|}-\frac{X(-1)}{|X(-1)|}\right|
$$

and since by $(3.8)$ and $M \ll 1$,

$$
|X(-1)| \geq\left|X\left(t_{+}\right)\right|-\left|X\left(t_{+}\right)-X(0)\right|-|X(0)-X(-1)| \geq 3-C M \geq 2,
$$

we thus get $\left|X\left(t_{+}\right)-R \frac{X(-1)}{|X(-1)|}\right| \lesssim\left|X\left(t_{+}\right)-X(-1)\right|$, so that

$$
W_{\partial B_{R}}^{2}\left(\bar{f}_{+, R}, \hat{g}_{+, R}\right) \lesssim \int_{\Omega_{+, R}}\left|X\left(t_{+}\right)-X(-1)\right|^{2} d \mathbb{P} .
$$

By the triangle inequality and the straightness of trajectories $X$ on $(0,1)$ this yields

$$
W_{\partial B_{R}}^{2}\left(\bar{f}_{+, R}, \hat{g}_{+, R}\right) \lesssim \int_{\Omega_{+, R}}\left(|X(1)-X(0)|^{2}+|X(0)-X(-1)|^{2}\right) d \mathbb{P} .
$$

By (3.24) this upgrades to

$$
\begin{aligned}
& \int_{3}^{4} W_{\partial B_{R}}^{2}\left(\bar{f}_{+, R}, \hat{g}_{+, R}\right) d R \\
& \lesssim \int_{\Omega_{5}}|X(1)-X(0)|\left(|X(1)-X(0)|^{2}+|X(0)-X(-1)|^{2}\right) d \mathbb{P} \\
& \stackrel{(3.8)}{\lesssim} M(E+D) .
\end{aligned}
$$


Using Lemma 2.10, we now show that we can choose $R$ such that $\bar{\rho}$ (recall (3.4)) is close to the Lebesgue measure in Wasserstein distance.

Lemma 3.3. We have

$$
\int_{3}^{4}\left(W_{B_{R}}^{2}\left(\bar{\rho}, \kappa_{R}\right)+\frac{1}{\kappa_{R}}\left(\kappa_{R}-1\right)^{2}\right) d R \lesssim E+D .
$$

Proof. From the first item in (3.1) and from 3.2 we have for all $t \in[0,1]$ that

$$
W_{B_{5}}^{2}\left(\rho_{t}^{\prime}, \kappa_{\mu}\right) \leq E+D,
$$

where the measure $0 \leq \rho_{t}^{\prime} \leq \rho_{t}$ is defined via

$$
\int \zeta d \rho_{t}^{\prime}=\int \zeta(X(t)) I(|X(-1)|<5) d \mathbb{P} .
$$

By (3.8), we obtain from 3.3 that $\rho_{t}^{\prime}=\rho_{t}$ on $B_{4}$. Hence, applying Lemma 2.10 with $\rho_{t}^{\prime}$ playing the role of $\mu$ yields (recall (2.3))

$$
\int_{3}^{4}\left(W_{B_{R}}^{2}\left(\rho_{t}, \kappa_{t}\right)+\frac{1}{\kappa_{t}}\left(\kappa_{t}-1\right)^{2}\right) d R \lesssim E+D
$$

which once integrated in time and thanks to the sub-additivity of $W^{2}$ gives the claim.

\subsubsection{Good radius for the construction}

For the construction part of the proof, we will need to introduce initial/terminal layers of size (multiples of) $\tau \ll 1$. We will treat a class of exceptional trajectories separately, namely exiting trajectories that exit too early and entering trajectories that enter too late:

$$
\delta \Omega_{R}:=\left(\Omega_{+, R} \cap\left\{t_{+} \leq 3 \tau\right\}\right) \cup\left(\Omega_{-, R} \cap\left\{t_{-} \geq 1-3 \tau\right\}\right) .
$$

These trajectories will be inserted into our construction of a competitor.

This means that we will have to pass (however only inside the construction part of the proof) to modified versions of the fluxes. We thus introduce the reduced sets of trajectories

$$
\Omega_{+, R}^{\prime}:=\Omega_{+, R} \backslash \delta \Omega_{R}, \quad \Omega_{-, R}^{\prime}:=\Omega_{-, R} \backslash \delta \Omega_{R}
$$

and then the reduced fluxes on $\partial B_{R} \times[0,1]$

$$
\int \zeta d f_{ \pm, R}^{\prime}=\int_{\Omega_{ \pm, R}^{\prime}} \zeta\left(X\left(t_{ \pm}\right), t_{ \pm}\right) d \mathbb{P} \quad \text { and } \quad f_{R}^{\prime}:=f_{+, R}^{\prime}-f_{-, R}^{\prime}
$$

from which $\bar{f}_{ \pm, R}^{\prime}$ and $\bar{f}_{R}^{\prime}$ are naturally defined as their time integral. Notice that $f_{R}^{\prime}$ is the flux created by all the trajectories which are not in $\delta \Omega_{R}$. We finally introduce the corresponding (constant in time) regularized fluxes:

$$
\int \zeta d \hat{g}_{+, R}^{\prime}=\int_{\Omega_{+, R}^{\prime}} \zeta\left(R \frac{X(-1)}{|X(-1)|}\right) d \mathbb{P}, \quad \int \zeta d \hat{g}_{-, R}^{\prime}=\int_{\Omega_{-, R}^{\prime}} \zeta\left(R \frac{X(2)}{|X(2)|}\right) d \mathbb{P}
$$


and

$$
\hat{g}_{R}^{\prime}:=\hat{g}_{+, R}^{\prime}-\hat{g}_{-, R}^{\prime}
$$

Arguing as in Lemma 3.2 , we obtain the following analogous estimates:

Lemma 3.4. With $M=(E+D)^{\frac{1}{d+2}}$, we have

$$
\begin{array}{r}
\int_{3}^{4} \int_{\partial B_{R}}\left(\hat{g}_{ \pm, R}^{\prime}\right)^{2} d R \lesssim E+D, \\
\int_{3}^{4} W_{\partial B_{R}}^{2}\left(\hat{g}_{ \pm, R}^{\prime}, \bar{f}_{ \pm, R}^{\prime}\right) d R \lesssim M(E+D) .
\end{array}
$$

Moreover,

$$
\int_{3}^{4} \int_{\partial B_{R}}\left(\hat{g}_{ \pm, R}^{\prime}-\hat{g}_{ \pm, R}\right)^{2} d R \lesssim \tau^{2} E+D
$$

Proof. The proofs of (3.31) and 3.32 are the same as the proofs of 3.19 and 3.20 so that we only need to comment on (3.33). The proof follows the lines of $(3.19)$. We focus once again only on the case with +-sign. Recall the definition (3.21) of $g_{+, R}$ and analogously define $g_{+, R}^{\prime}$ so that

$$
\int \zeta d\left(g_{+, R}-g_{+, R}^{\prime}\right)=\int_{\Omega_{+, R} \cap \delta \Omega_{R}} \zeta(X(-1)) d \mathbb{P} .
$$

Since by definition 3.26 of $\delta \Omega_{R}$

$$
\Omega_{+, R} \cap \delta \Omega_{R}=\left(\Omega_{+, R} \cap\left\{t_{+} \leq 3 \tau\right\}\right) \cup\left(\Omega_{+, R} \cap\left\{t_{-} \geq 1-3 \tau\right\}\right),
$$

using the piecewise straightness of trajecteries in form of $\left|X\left(t_{+}\right)-X(0)\right| \leq 3 \tau \mid X(1)-$ $X(0) \mid$, the analog of $(3.23)$ becomes

$$
\begin{aligned}
\int|R-| x|| d\left(g_{+, R}-g_{+, R}^{\prime}\right) & \leq \int_{\Omega_{+, R} \cap\left\{t_{+} \leq 3 \tau\right\}}(3 \tau|X(1)-X(0)|+|X(0)-X(-1)|) d \mathbb{P} \\
+ & \int_{\Omega_{+, R} \cap\left\{t_{-} \geq 1-3 \tau\right\}}(|X(1)-X(0)|+|X(0)-X(-1)|) d \mathbb{P} .
\end{aligned}
$$

Since for $X \in \Omega_{+, R} \cap\left\{t_{+} \leq 3 \tau\right\}$ we have $\min _{[0,3 \tau]}|X|<R \leq|X(3 \tau)|$ and therefore, the analog of (3.24) for the first term on the right-hand side turns into

$$
\int_{0}^{\infty} I\left(X \in \Omega_{+, R} \cap\left\{t_{+} \leq 3 \tau\right\}\right) d R \lesssim \tau|X(1)-X(0)| .
$$

We now estimate the second term on the right-hand side and note that for $X \in$ $\Omega_{+, R} \cap\left\{t_{-} \geq 1-3 \tau\right\}$ we have by definitions (3.6) and $(3.9)$ that $|X(1)|,|X(1-3 \tau)| \geq R$ while $\min _{[1-3 \tau, 1]}|X|<R$. By the straightness of trajectories and the $L^{\infty}$ bound $(3.8)$ in form of

$$
\max _{t \in[1-3 \tau, 1]}|X(1)-X(t)| \leq 3 \tau|X(1)-X(0)| \leq 3 \tau M
$$


in conjunction with $R \geq 3$, we always have $\min _{[1-3 \tau, 1]}|X| \geq 2$. Hence the elementary inequality $\min \{|X(1)|,|X(1-3 \tau)|\}-\min _{[1-3 \tau, 1]}|X| \leq \frac{|X(1)-X(1-3 \tau)|^{2}}{8 \min _{[1-3 \tau, 1]}|X|}$ implies

$$
\begin{gathered}
\int_{3}^{4} I\left(X \in \Omega_{+, R} \cap\left\{t_{-} \geq 1-3 \tau\right\}\right) d R \leq \frac{1}{16}|X(1)-X(1-3 \tau)|^{2} \\
\stackrel{3.34}{\leq} \frac{3}{16} \tau^{2} M|X(1)-X(0)| \ll \tau^{2}|X(1)-X(0)|,
\end{gathered}
$$

(in fact, this contribution will be of higher order). Summing up, we obtain

$$
\begin{aligned}
& \int_{3}^{4} \int(R-|x|) d\left(g_{+, R}-g_{+, R}^{\prime}\right) d \mathbb{P} \\
& \lesssim \int \tau|X(1)-X(0)|(\tau|X(1)-X(0)|+|X(0)-X(-1)|) d \mathbb{P} \\
& +\int \tau^{2}|X(1)-X(0)|(|X(1)-X(0)|+|X(0)-X(-1)|) d \mathbb{P}
\end{aligned}
$$

so that 3.33 follows from Young's inequality in form of $2 \tau \mid X(1)-X(0) \| X(0)-$ $X(-1)\left|\leq \tau^{2}\right| X(1)-\left.X(0)\right|^{2}+|X(0)-X(-1)|^{2}$ and $(3.2)$.

Define the sets of generic trajectories (recall (3.7) and (3.26)) that on $[0,1]$ start in $B_{R}$ and do not exit too early or that end in $B_{R}$ and do not enter too late, respectively,

$$
\begin{aligned}
& \Omega_{0, R}:=\quad\left(\Omega_{R} \backslash \delta \Omega_{R}\right) \cap\{|X(0)|<R\}=\left\{|X(0)|<R \text { and } t_{+}>3 \tau\right\}, \\
& \Omega_{1, R}:=\left(\Omega_{R} \backslash \delta \Omega_{R}\right) \cap\{|X(1)|<R\}=\left\{|X(1)|<R \text { and } t_{-}<1-3 \tau\right\} .
\end{aligned}
$$

In line with (3.1), we define the measures in $B_{R}$

$$
\int \zeta d \mu_{R}^{\prime}=\int_{\Omega_{0, R}} \zeta(X(0)) d \mathbb{P}, \quad \int \zeta d \lambda_{R}^{\prime}=\int_{\Omega_{1, R}} \zeta(X(1)) d \mathbb{P},
$$

which correspond to where the trajectories which are not in $\delta \Omega_{R}$ start and end provided they start or end in $B_{R}$. An important step in the construction is to connect over a time $\tau$ the measure $0 \leq \mu_{R}^{\prime} \leq \mu$ to a constant $\kappa_{\mu_{R}^{\prime}}$ and the measure $0 \leq \lambda_{R}^{\prime} \leq \lambda$ to another constant $\kappa_{\lambda_{R}^{\prime}}$

Lemma 3.5. We have

$$
\begin{array}{r}
\int_{3}^{4}\left(W_{B_{R}}^{2}\left(\mu_{R}^{\prime}, \kappa_{\mu_{R}^{\prime}}\right)+\frac{1}{\kappa_{\mu_{R}^{\prime}}}\left(\kappa_{\mu_{R}^{\prime}}-1\right)^{2}+W_{B_{R}}^{2}\left(\lambda_{R}^{\prime}, \kappa_{\lambda_{R}^{\prime}}\right)+\frac{1}{\kappa_{\lambda_{R}^{\prime}}}\left(\kappa_{\lambda_{R}^{\prime}}-1\right)^{2}\right) d R \\
\lesssim \tau^{2} E+D .
\end{array}
$$

Proof. By symmetry, we may restrict to $\mu_{R}^{\prime}$. The statement follows from combining the outcome of the four steps below.

STEP 1. Introducing the measure $0 \leq g_{0, R} \leq \kappa_{\mu}$ that captures where the generic trajectories $X \in \Omega_{0, R}$ start at time $t=-1$, that is,

$$
\int \zeta d g_{0, R}=\int_{\Omega_{0, R}} \zeta(X(-1)) d \mathbb{P}
$$


we claim that its deviation from the characteristic function $\kappa_{\mu} I\left(B_{R}\right)$ is small (on average in $R \in(3,4))$ :

$$
\begin{gathered}
\int_{3}^{4} \int_{B_{R}}(R-|x|) d\left(\kappa_{\mu}-g_{0, R}\right)+\int_{B_{R}^{c}}(|x|-R) d g_{0, R} d R \\
\lesssim \tau^{2} E+D .
\end{gathered}
$$

The argument is a refinement of $\left(3.22\right.$ in Lemma 3.2 . By $\Omega_{0, R} \subset\{|X(0)|<R\}$, cf. (3.35), we have on the one hand

$$
\int_{B_{R}^{c}}(|x|-R) d g_{0, R} \leq \int_{|X(0)|<R \leq|X(-1)|}|X(-1)|-|X(0)| d \mathbb{P} .
$$

Note that by (3.1) and (3.38), we have on the other hand

$$
\int_{B_{R}}(R-|x|) d\left(\kappa_{\mu}-g_{0, R}\right)=\int_{\Omega_{0, R}^{c}}(R-|X(-1)|)_{+} d \mathbb{P}
$$

and thus, since $\Omega_{0, R}^{c} \subset\{|X(0)| \geq R\} \cup\left\{t_{+} \leq 3 \tau\right\} \subset\{\max (|X(0)|,|X(3 \tau)|) \geq R\}$, cf. (3.35) and (3.9),

$$
\begin{aligned}
& \int_{B_{R}}(R-|x|) d\left(\kappa_{\mu}-g_{0, R}\right) \\
& \leq \int_{|X(-1)|<R \leq \max (|X(0)|,|X(3 \tau)|)} \max (|X(0)|,|X(3 \tau)|)-|X(-1)| d \mathbb{P} .
\end{aligned}
$$

Integrating these two estimates in $R$ and using the straightness of $X$ on $[0,1]$ in form of

$$
\max (|X(0)|,|X(3 \tau)|)-|X(-1)| \leq 3 \tau|X(1)-X(0)|+|X(0)-X(-1)|,
$$

we obtain 3.39 ).

STEP 2. We claim that the total mass density $\kappa_{\mu_{R}^{\prime}}=\frac{\mu_{R}^{\prime}\left(B_{R}\right)}{\left|B_{R}\right|}$ is close to 1 on average:

$$
\int_{3}^{4} \frac{1}{\kappa_{\mu_{R}^{\prime}}}\left(\kappa_{\mu_{R}^{\prime}}-1\right)^{2} d R \lesssim \tau^{2} E+D
$$

Indeed, from definitions (3.36) and 3.38 we obtain $\mu_{R}^{\prime}\left(B_{R}\right)=\mathbb{P}\left(\Omega_{0, R}\right)=\int d g_{0, R}$ which we write as

$$
\left|B_{R}\right|\left(\kappa_{\mu_{R}^{\prime}}-\kappa_{\mu}\right)=\int_{B_{R}}\left(g_{0, R}-\kappa_{\mu}\right)+\int_{B_{R}^{c}} g_{0, R} .
$$

We now appeal to 2.13) in Lemma 2.3 , and use $0 \leq g_{0, R} \leq \kappa_{\mu} \sim 1$ to obtain

$$
\left(\kappa_{\mu_{R}^{\prime}}-\kappa_{\mu}\right)^{2} \lesssim \int_{B_{R}}(R-|x|)\left(\kappa_{\mu}-g_{0, R}\right)+\int_{B_{R}^{c}}(|x|-R) g_{0, R} .
$$


Thus 3.40 follows from $3.39, \frac{1}{\kappa_{\mu}}\left(\kappa_{\mu}-1\right)^{2} \leq D \ll 1$ and the fact that by 3.8

$$
\kappa_{\mu_{R}^{\prime}} \sim 1 \quad \forall R \in(3,4) \text {. }
$$

STEP 3. We claim the inequality

$$
\begin{aligned}
& W_{B_{R}}\left(\mu_{R}^{\prime}, \kappa_{\mu_{R}^{\prime}}\right) \\
& \leq D^{\frac{1}{2}}+\left(M \int_{B_{R}^{c}}(|x|-R) d g_{0, R}\right)^{\frac{1}{2}}+W_{\bar{B}_{R}}\left(g_{0, R}+\hat{g}_{0, R}, \kappa_{\mu_{R}^{\prime}}\right),
\end{aligned}
$$

where in line with $(3.29), \hat{g}_{0, R}$ is defined as the projection of $g_{0, R}$ from $B_{R}^{c}$ onto $\partial B_{R}$, that is,

$$
\int \zeta d \hat{g}_{0, R}=\int_{B_{R}^{c}} \zeta\left(R \frac{x}{|x|}\right) d g_{0, R}
$$

Indeed, we start from the triangle inequality

$$
\begin{aligned}
& W_{B_{R}}\left(\mu_{R}^{\prime}, \kappa_{\mu_{R}^{\prime}}\right) \\
& \leq W\left(\mu_{R}^{\prime}, g_{0, R}\right)+W\left(g_{0, R}, g_{0, R} I\left(B_{R}\right)+\hat{g}_{0, R}\right)+W_{\bar{B}_{R}}\left(g_{0, R}+\hat{g}_{0, R}, \kappa_{\mu_{R}^{\prime}}\right) .
\end{aligned}
$$

Clearly, by the definitions (3.36) and (3.38), it follows from (3.2) that $W\left(\mu_{R}^{\prime}, g_{0, R}\right) \leq$ $D^{\frac{1}{2}}$. By monotonicity, $W\left(g_{0, R}, g_{0, R} I\left(B_{R}\right)+\hat{g}_{0, R}\right) \leq W\left(g_{0, R} I\left(B_{R}^{c}\right), \hat{g}_{0, R}\right)$; in view of definition (3.43) and a purely radial coupling we obtain $W^{2}\left(g_{0, R} I\left(B_{R}^{c}\right), \hat{g}_{0, R}\right) \leq$ $\int_{B_{R}^{c}}(|x|-R)^{2} d g_{0, R}$; by definition 3.38 and the $L^{\infty}$-bound $(3.8), g_{0, R}$ is supported in $B_{R+M}$, so that we obtain the claimed inequality on this term, too.

STEP 4. We claim that the last term in 3.42 is controlled as follows

$$
\begin{aligned}
& W_{\bar{B}_{R}}^{2}\left(g_{0, R}+\hat{g}_{0, R}, \kappa_{\mu_{R}^{\prime}}\right) \\
& \lesssim \int_{B_{R}}(R-|x|) d\left(\kappa_{\mu}-g_{0, R}\right)+\int_{B_{R}^{c}}(|x|-R) d g_{0, R} .
\end{aligned}
$$

We follow the argument from Lemma 2.10. We first appeal to 2.31) in Lemma 2.7 to reduce to an estimate of $W_{\bar{B}_{R}}^{2}\left(g_{0, R}+\hat{g}_{0, R}+\kappa_{\mu_{R}^{\prime}}, 2 \kappa_{\mu_{R}^{\prime}}\right)$. We then argue as for 2.51 to reduce further to an estimate of $\frac{1}{\kappa_{\mu_{R}^{\prime}}} \int_{B_{R}}|\nabla \varphi|^{2}$, where $\nabla \varphi$ is determined through

$$
\Delta \varphi=c-g_{0, R} \text { in } B_{R} \quad \text { and } \quad \nu \cdot \nabla \varphi=\hat{g}_{0, R} \text { on } \partial B_{R},
$$

and $c$ is such that the problem is solvable. By (3.41) we just need to control $\int_{B_{R}}|\nabla \varphi|^{2}$, for which we appeal to the energy estimate (2.11) in conjunction with 2.13 .

\subsubsection{Choice of the good radius and definition of $\Phi$ and $\phi$}

We can finally choose a good radius. We refer to (3.4) for the definition of $\bar{\rho}$, to (3.16) for the definition of $\hat{g}_{ \pm, R}$, to 3.17) for the definition of $\bar{f}_{ \pm, R}$, to (3.26) for the definition of $\delta \Omega_{R}$, to 3.28 for the definition of $\bar{f}_{ \pm, R}^{\prime}$, to 3.29 for the definition of $\hat{g}_{ \pm, R}^{\prime}$, and to 3.36 for the definitions of $\mu_{R}^{\prime}$ and $\lambda_{R}^{\prime}$. 
Proposition 3.6. Let $\pi$ be an optimal coupling between $\mu$ and $\lambda$ such that $E+D \ll 1$. Recall the definition $M=(E+D)^{\frac{1}{d+2}}$. Then, there exists a set of radii $R \in(3,4)$ of positive measure such that we have on the one hand:

$$
\begin{aligned}
& W_{B_{R}}^{2}\left(\mu, \kappa_{\mu, R}\right)+W_{B_{R}}^{2}\left(\lambda, \kappa_{\lambda, R}\right) \lesssim D, \\
& W_{B_{R}}^{2}(\bar{\rho}, \kappa)+\frac{1}{\kappa}(\kappa-1)^{2} \lesssim E+D, \\
& W_{\partial B_{R}}^{2}\left(\bar{f}_{ \pm, R}, \hat{g}_{ \pm, R}\right) \lesssim M(E+D), \\
& \int_{\partial B_{R}} \hat{g}_{ \pm, R}^{2} \lesssim E+D
\end{aligned}
$$

and on the other hand:

$$
\begin{aligned}
& \int_{\partial B_{R}}\left(\hat{g}_{ \pm, R}^{\prime}\right)^{2} \lesssim E+D \\
& W_{\partial B_{R}}^{2}\left(\hat{g}_{ \pm, R}^{\prime}, \bar{f}_{ \pm, R}^{\prime}\right) \lesssim M(E+D), \\
& \int_{\partial B_{R}}\left(\hat{g}_{ \pm, R}^{\prime}-\hat{g}_{ \pm}\right)^{2} \lesssim \tau^{2} E+D, \\
& W_{B_{R}}^{2}\left(\mu_{R}^{\prime}, \kappa_{\mu_{R}^{\prime}}\right)+\frac{1}{\kappa_{\mu_{R}^{\prime}}}\left(\kappa_{\mu_{R}^{\prime}}-1\right)^{2}+W_{B_{R}}^{2}\left(\lambda_{R}^{\prime}, \kappa_{\lambda_{R}^{\prime}}\right)+\frac{1}{\kappa_{\lambda_{R}^{\prime}}}\left(\kappa_{\lambda_{R}^{\prime}}-1\right)^{2} \\
& \quad \lesssim \tau^{2} E+D .
\end{aligned}
$$

Proof. Estimate (3.44) follows from an application of Lemma 2.10 both to $\mu$ and $\lambda$; (3.45) is obtained from (3.25) of Lemma 3.3. (3.46) and (3.47) follow from Lemma 3.2 (3.48), (3.49) and (3.50) are a by-product of Lemma 3.4 and (3.51) follows from Lemma 3.5 .

From now on we fix a radius $R \in(3,4)$ satisfying all the conditions of Proposition 3.6 .

Convention: we drop the notational dependence in $R$ when it does not lead to confusion (and write in particular $f$ for $f_{R}, \hat{g}$ for $\hat{g}_{R}$ etc...).

We recall the definition of $\Phi$ from (1.15), the solution to the Poisson equation with non-regularized flux boundary conditions (recall the definition $(3.18)$ of $\bar{f}$ )

$$
\Delta \Phi=c \text { in } B_{R} \quad \nu \cdot \nabla \Phi=\bar{f} \text { on } \partial B_{R}
$$

and define the function $\phi$, which is the solution to the same Poisson equation but with regularized flux boundary conditions

$$
\Delta \phi=c \text { in } B_{R} \quad \nu \cdot \nabla \phi=\hat{g} \text { on } \partial B_{R} .
$$

We normalize $\Phi$ and $\phi$ by imposing $\int_{B_{R}} \Phi=\int_{B_{R}} \phi=0$. As already explained, we will first prove the harmonic approximation result with the function $\phi$ and then prove that we can replace it by the function $\Phi$. 


\subsection{Eulerian version of the harmonic approximation re- sult}

The aim of this section is to prove the Eulerian version of the harmonic approximation result, that is Proposition 1.6, which we recall for the reader's convenience:

Proposition 1.6. For every $0<\tau \ll 1$, there exist positive constants $\varepsilon(\tau)>0$ and $C(\tau)>0$ such that if $E+D \leq \varepsilon(\tau)$, then there exists $R \in(3,4)$ such that if $\Phi$ is defined through 3.52,

$$
\int_{B_{2}} \int_{0}^{1} \frac{1}{\rho}|j-\rho \nabla \Phi|^{2} \leq \tau E+C(\tau) D .
$$

Moreover,

$$
\sup _{B_{2}}\left|\nabla^{2} \Phi\right|^{2}+\sup _{B_{2}}|\nabla \Phi|^{2} \lesssim E+D .
$$

Most of the work will be devoted to the proof of the analog statement but with $\Phi$ replaced by $\phi$.

Proposition 3.7. For every $0<\tau \ll 1$, there exist positive constants $\varepsilon(\tau)>0$ and $C(\tau)>0$ such that if $E+D \leq \varepsilon(\tau)$, then there exists $R \in(3,4)$ such that if $\phi$ is defined through (3.53),

$$
\int_{B_{2}} \int_{0}^{1} \frac{1}{\rho}|j-\rho \nabla \phi|^{2} \leq \tau E+C(\tau) D .
$$

Proof. The proof is a direct combination of the orthogonality Lemma 1.7 and the construction Lemma 1.8 which are contained in the next two subsections together with (3.14) and the choice of parameters $r=\tau$.

Before coming to the proof of Lemma 1.7 and Lemma 1.8, let us derive Proposition 1.6 from Proposition 3.7

Proof of Proposition 1.6. Let $\varphi_{ \pm}$be the solution of

$$
\Delta \varphi_{ \pm}=0 \text { in } B_{R} \quad \text { and } \quad \nu \cdot \nabla \varphi_{ \pm}=\bar{f}_{ \pm}-\hat{g}_{ \pm} \text {on } \partial B_{R}
$$

with $\int_{B_{R}} \varphi_{ \pm}=0$ so that $\Phi-\phi=\varphi_{+}-\varphi_{-}$. Applying Lemma 2.4 to $\varphi_{ \pm}$, we find that for every $0<\alpha<1$,

$$
\begin{aligned}
\sup _{B_{2}}\left|\nabla^{2}(\Phi-\phi)\right|+\sup _{B_{2}}|\nabla(\Phi-\phi)| & \lesssim W_{\partial B_{R}}^{\alpha}\left(\bar{f}_{ \pm}, \hat{g}_{ \pm}\right)\left[\hat{g}_{ \pm}\left(\partial B_{R}\right)\right]^{1-\frac{\alpha}{2}} \\
& \stackrel{3.46}{\lesssim}(M(E+D))^{\frac{\alpha}{2}}\left(\int_{\partial B_{R}} \hat{g}_{ \pm}^{2}\right)^{\frac{1}{2}-\frac{\alpha}{4}} \\
& \stackrel{3.47}{\lesssim}(M(E+D))^{\frac{\alpha}{2}}(E+D)^{\frac{1}{2}-\frac{\alpha}{4}} \\
& \lesssim M^{\frac{\alpha}{2}}(E+D)^{\frac{1}{2}+\frac{\alpha}{4}}
\end{aligned}
$$


Combining this with (2.7) applied to $\phi$ and (3.47) we first obtain (3.55). This also yields

$$
\begin{aligned}
\int_{B_{2}} \int_{0}^{1} \frac{1}{\rho}|j-\rho \nabla \Phi|^{2} & \lesssim \int_{B_{2}} \int_{0}^{1} \frac{1}{\rho}|j-\rho \nabla \phi|^{2}+\int_{B_{2}}|\nabla \Phi-\nabla \phi|^{2} d \bar{\rho} \\
& \stackrel{3.56)}{\lesssim} \tau E+C(\tau) D+M^{\alpha}(E+D)^{1+\frac{\alpha}{2}} \int_{B_{2}} d \bar{\rho} \\
& \stackrel{3.5}{\lesssim} \tau E+C(\tau) D+M^{\alpha}(E+D)^{1+\frac{\alpha}{2}},
\end{aligned}
$$

which concludes the proof of 3.54 .

\subsubsection{Approximate orthogonality}

In this section we prove the approximate orthogonality property, that is Lemma 1.7 which we recall for the reader's conveniencevii:

Lemma 1.7 (Orthogonality). For every $0<r \ll 1$, there exist $\varepsilon(r)>0$ and $C(r)>0$ such that if $E+D \leq \varepsilon$,

$$
\int_{B_{2}} \int_{0}^{1} \frac{1}{\rho}|j-\rho \nabla \phi|^{2}-\left(\int_{B_{R}} \int_{0}^{1} \frac{1}{\rho}|j|^{2}-\int_{B_{R}}|\nabla \phi|^{2}\right) \leq r E+C D .
$$

Proof. Let $0<r \ll 1$ be given and let us recall the notation from Lemma 2.1. If $\hat{g}$ is given by (3.15), we let $\hat{g}_{r}$ be the convolution with a smooth convolution kernel at scale $r$ (on $\partial B_{R}$ ) of $\hat{g}$. We then let $\phi^{r}$ with $\int_{B_{R}} \phi^{r}=0$ be the solution of

$$
\Delta \phi^{r}=c \text { in } B_{R} \quad \text { and } \quad \nu \cdot \nabla \phi^{r}=\hat{g}_{r} \text { on } \partial B_{R} .
$$

We first prove (3.57) with $\phi^{r}$ instead of $\phi$ and then get rid of this regularization.

STEP 1. We prove that

$$
\begin{aligned}
\int_{B_{R}} \int_{0}^{1} \frac{1}{\rho}\left|j-\rho \nabla \phi^{r}\right|^{2} & -\left(\int_{B_{R}} \int_{0}^{1} \frac{1}{\rho}|j|^{2}-\int_{B_{R}}\left|\nabla \phi^{r}\right|^{2}\right) \\
& \lesssim r E+\frac{1}{r} D+\frac{1}{r^{d+2}} D^{2}+\frac{1}{r^{d+1}} E^{2}+\frac{1}{r^{\frac{d+1}{2}}} M^{\frac{1}{2}}(E+D)^{\frac{5}{4}}
\end{aligned}
$$

Our argument relies on the formula

$$
\begin{aligned}
\int_{B_{R}} \int_{0}^{1} \frac{1}{\rho}\left|j-\rho \nabla \phi^{r}\right|^{2}= & \int_{B_{R}} \int_{0}^{1} \frac{1}{\rho}|j|^{2}-\int_{B_{R}}\left|\nabla \phi^{r}\right|^{2} \\
& +2 \int_{B_{R}} \phi^{r} d(\mu-\lambda)+2 \int_{\partial B_{R}} \phi^{r} d\left(\hat{g}_{r}-\bar{f}\right) \\
& +\int_{B_{R}}\left|\nabla \phi^{r}\right|^{2}(d \bar{\rho}-d x),
\end{aligned}
$$

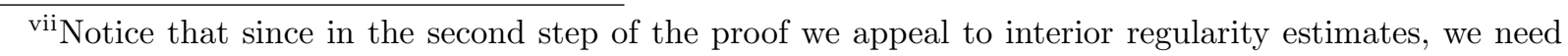
to go from $B_{R}$ in the term between parenthesis on the left-hand side of (3.57) to the smaller ball $B_{2}$ for the first term on the left-hand side of 3.57 
where we recall the definitions of $\bar{f}$ in $(3.18)$ and $\bar{\rho}$ in (3.4). This formula is obtained by expanding the square and combining (3.12) with (3.58), both tested with $\phi^{r}$ (which is smooth), and using that $\int_{B_{R}} \phi^{r}=0$.

SteP 1.1. We first estimate $\int_{B_{R}} \phi^{r} d(\mu-\lambda)$. For this, using again $\int_{B_{R}} \phi^{r}=0$, we write it as (recall (3.44) for the definition of $\kappa_{\mu, R}$ and $\kappa_{\lambda, R}$ )

$$
\int_{B_{R}} \phi^{r} d(\mu-\lambda)=\int_{B_{R}} \phi^{r}\left(d \mu-\kappa_{\mu, R} d x\right)+\int_{B_{R}} \phi^{r}\left(d \lambda-\kappa_{\lambda, R} d x\right)
$$

and estimate using $\kappa_{\mu, R} \sim 1$ in the second step

$$
\begin{aligned}
& \left|\int_{B_{R}} \phi^{r}\left(d \mu-\kappa_{\mu, R} d x\right)\right| \\
& \stackrel{2.32}{\leq}\left(\kappa_{\mu, R} \int_{B_{R}}\left|\nabla \phi^{r}\right|^{2}\right)^{\frac{1}{2}} W_{B_{R}}\left(\mu, \kappa_{\mu, R}\right)+\frac{1}{2} \sup _{B_{R}}\left|\nabla^{2} \phi^{r}\right| W_{B_{R}}^{2}\left(\mu, \kappa_{\mu, R}\right) \\
& \underbrace{\infty 2.77}_{\lesssim}\left(\int_{\partial B_{R}} \hat{g}_{r}^{2}\right)^{\frac{1}{2}} W_{B_{R}}\left(\mu, \kappa_{\mu, R}\right)+\frac{1}{r}\left(\frac{1}{r^{d-1}} \int_{\partial B_{R}} \hat{g}_{r}^{2}\right)^{\frac{1}{2}} W_{B_{R}}^{2}\left(\mu, \kappa_{\mu, R}\right) \\
& \underbrace{\lesssim}_{\lesssim}(E+D)^{\frac{1}{2}} D^{\frac{1}{2}}+\frac{1}{r^{\frac{d+1}{2}}}(E+D)^{\frac{1}{2}} D \\
& \lesssim r E+\frac{1}{r} D+\frac{1}{r^{d+2}} D^{2} .
\end{aligned}
$$

The term $\int_{B_{R}} \phi^{r}\left(d \lambda-\kappa_{\lambda, R} d x\right)$ may be estimated analogously.

STEP 1.2. We then estimate $\int_{\partial B_{R}} \phi^{r} d\left(\hat{g}_{r}-\bar{f}\right)$. For this we split this term as follows

$$
\int_{\partial B_{R}} \phi^{r} d\left(\hat{g}_{r}-\bar{f}\right)=\int_{\partial B_{R}}\left(\phi_{r}^{r}-\phi^{r}\right) d \hat{g}+\int_{\partial B_{R}} \phi^{r} d(\hat{g}-\bar{f}) .
$$

On the one hand we have

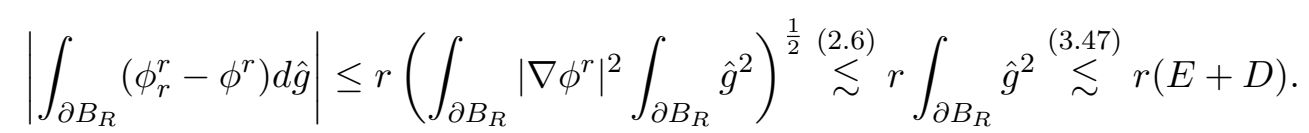

On the other hand, treating separately the positive and negative parts and arguing as for (2.32), we obtain

$$
\begin{aligned}
& \left|\int_{\partial B_{R}} \phi^{r} d\left(\hat{g}_{ \pm}-\bar{f}_{ \pm}\right)\right| \\
& \leq \sup _{\partial B_{R}}\left|\nabla_{\tan } \phi^{r}\right|\left(\int_{\partial B_{R}} \hat{g}_{ \pm}\right)^{\frac{1}{2}} W_{\partial B_{R}}\left(\bar{f}_{ \pm}, \hat{g}_{ \pm}\right)+\frac{1}{2} \sup _{\partial B_{R}}\left|\nabla_{t a n}^{2} \phi^{r}\right| W_{\partial B_{R}}^{2}\left(\bar{f}_{ \pm}, \hat{g}_{ \pm}\right) \\
& \stackrel{\underbrace{2.4})}{\lesssim} \frac{1}{r^{\frac{d-1}{2}}}\left(\int_{\partial B_{R}} \hat{g}_{ \pm}^{2}\right)^{\frac{3}{4}} W_{\partial B_{R}}\left(\bar{f}_{ \pm}, \hat{g}_{ \pm}\right)+\frac{1}{r}\left(\frac{1}{r^{d-1}} \int_{\partial B_{R}} \hat{g}_{ \pm}^{2}\right)^{\frac{1}{2}} W_{\partial B_{R}}^{2}\left(\bar{f}_{ \pm}, \hat{g}_{ \pm}\right) \\
& \qquad \underset{r^{3.46} \& \sqrt{3.47}}{\lesssim} \frac{1}{r^{\frac{d-1}{2}}} M^{\frac{1}{2}}(E+D)^{\frac{5}{4}}+\frac{1}{r^{\frac{d+1}{2}}} M(E+D)^{\frac{3}{2}} \lesssim \frac{1}{r^{\frac{d+1}{2}}} M^{\frac{1}{2}}(E+D)^{\frac{5}{4}}
\end{aligned}
$$


STEP 1.3. We finally estimate $\int_{B_{R}}\left|\nabla \phi^{r}\right|^{2}\left(d \bar{\rho}-d x\right.$ ). Let $\zeta:=\left|\nabla \phi^{r}\right|^{2}$ (which is smooth) so that

$$
\begin{aligned}
& \left|\int_{B_{R}} \zeta(d \bar{\rho}-d x)\right| \\
& \leq\left|\int_{B_{R}} \zeta(d \bar{\rho}-\kappa d x)\right|+|\kappa-1| \int_{B_{R}} \zeta \\
& \stackrel{2.32}{\leq}\left(\int_{B_{R}}|\nabla \zeta|^{2}\right)^{\frac{1}{2}} W_{B_{R}}(\bar{\rho}, \kappa)+\frac{1}{2} \sup _{B_{R}}\left|\nabla^{2} \zeta\right| W_{B_{R}}^{2}(\bar{\rho}, \kappa)+|\kappa-1| \int_{B_{R}} \zeta \\
& \stackrel{\sqrt[3.45]{\lesssim}}{\lesssim}\left(\int_{B_{R}}|\nabla \zeta|^{2}\right)^{\frac{1}{2}}(E+D)^{\frac{1}{2}}+\sup _{B_{R}}\left|\nabla^{2} \zeta\right|(E+D)+\left(\int_{B_{R}} \zeta\right)(E+D)^{\frac{1}{2}}
\end{aligned}
$$

Since

$$
\begin{aligned}
& \int_{B_{R}} \zeta=\int_{B_{R}}\left|\nabla \phi^{r}\right|^{2} \stackrel{\sqrt[2.7]{\lesssim}}{\lesssim} \int_{\partial B_{R}} \hat{g}^{2} \stackrel{\sqrt[3.47]{\lesssim}}{\lesssim} E+D, \\
& \left(\int_{B_{R}}|\nabla \zeta|^{2}\right)^{\frac{1}{2}} \lesssim \sup _{B_{R}}\left|\nabla^{2} \phi^{r}\right|\left(\int_{B_{R}}\left|\nabla \phi^{r}\right|^{2}\right)^{\frac{1}{2}} \\
& \stackrel{2.4}{\lesssim} \frac{1}{\lesssim} \frac{1.7}{r^{\frac{d+1}{2}}} \int_{\partial B_{R}} \hat{g}^{2} \stackrel{\sqrt[3.47]{\lesssim}}{\lesssim} \frac{1}{r^{\frac{d+1}{2}}}(E+D),
\end{aligned}
$$

and

$$
\begin{aligned}
\sup _{B_{R}}\left|\nabla^{2} \zeta\right| & \lesssim \sup _{B_{R}}\left|\nabla \phi^{r}\right| \sup _{B_{R}}\left|\nabla^{3} \phi^{r}\right|+\sup _{B_{R}}\left|\nabla^{2} \phi^{r}\right|^{2} \\
& \stackrel{2.4}{\lesssim} \frac{1}{r^{d+1}} \int_{\partial B_{R}} \hat{g}^{2} \stackrel{3.47}{\lesssim} \frac{1}{r^{d+1}}(E+D),
\end{aligned}
$$

we conclude that

$$
\begin{aligned}
\left.\left|\int_{B_{R}}\right| \nabla \phi^{r}\right|^{2}(d \bar{\rho}-d x) \mid \lesssim \frac{1}{r^{\frac{d+1}{2}}}(E+D)^{\frac{3}{2}}+\frac{1}{r^{d+1}}(E+D)^{2}+(E+D)^{\frac{3}{2}} \\
\\
\lesssim \frac{1}{r^{\frac{d+1}{2}}}(E+D)^{\frac{3}{2}}+\frac{1}{r^{d+1}}(E+D)^{2}
\end{aligned}
$$

Note that because of $M=(E+D)^{\frac{1}{d+2}}$, also the first right-hand side term is contained in the last right-hand side term of 3.59 .

Combining Step 1.1, Step 1.2 and Step 1.3 together, we obtain (3.59).

SteP 2. We now pass from $\phi^{r}$ to $\phi$. Using the identity

$$
\frac{1}{\rho}|j-\rho \nabla \phi|^{2}-\frac{1}{\rho}\left|j-\rho \nabla \phi^{r}\right|^{2}=2 j \cdot \nabla\left(\phi-\phi^{r}\right)+\rho\left(|\nabla \phi|^{2}-\left|\nabla \phi^{r}\right|^{2}\right),
$$


and Cauchy-Schwarz's inequality, we first obtain

$$
\begin{aligned}
& \int_{B_{2}} \int_{0}^{1} \frac{1}{\rho}|j-\rho \nabla \phi|^{2}-\int_{B_{2}} \int_{0}^{1} \frac{1}{\rho}\left|j-\rho \nabla \phi^{r}\right|^{2} \\
& \lesssim\left(\int_{B_{2}} d \bar{\rho} \int_{B_{2}} \int_{0}^{1} \frac{1}{\rho}|j|^{2}\right)^{\frac{1}{2}} \sup _{B_{2}}\left|\nabla\left(\phi-\phi^{r}\right)\right| \\
& +\left(\sup _{B_{2}}\left|\nabla\left(\phi-\phi^{r}\right)\right|\right)\left(\sup _{B_{2}}|\nabla \phi|+\left|\nabla \phi^{r}\right|\right) \int_{B_{2}} d \bar{\rho} \\
& \stackrel{2.5) \& 2.55 \& 2.7}{\lesssim} r E^{\frac{1}{2}}\left(\int_{\partial B_{R}} \hat{g}^{2}\right)^{\frac{1}{2}}+r \int_{\partial B_{R}} \hat{g}^{2} \\
& \stackrel{\sqrt[3.47]{\lesssim}}{\lesssim} r(E+D) \text {. }
\end{aligned}
$$

Now by (3.53) and (3.58), we also have

$$
\begin{aligned}
\int_{B_{R}}|\nabla \phi|^{2}-\int_{B_{R}}\left|\nabla \phi^{r}\right|^{2} & =\int_{\partial B_{R}}\left(\phi+\phi^{r}\right)\left(\hat{g}-\hat{g}_{r}\right) \\
& =\int_{\partial B_{R}}\left[\left(\phi+\phi^{r}\right)-\left(\phi+\phi^{r}\right)_{r}\right] \hat{g} \\
& \lesssim r\left(\int_{\partial B_{R}}\left|\nabla\left(\phi+\phi^{r}\right)\right|^{2}\right)^{\frac{1}{2}}\left(\int_{\partial B_{R}} \hat{g}^{2}\right)^{\frac{1}{2}} \\
& \stackrel{\lesssim}{\lesssim} r(E+D),
\end{aligned}
$$

so that combined with (3.59) and the obvious $\int_{B_{2}} \int_{0}^{1} \frac{1}{\rho}\left|j-\rho \nabla \phi^{r}\right|^{2} \leq \int_{B_{R}} \int_{0}^{1} \frac{1}{\rho} \mid j-$ $\left.\rho \nabla \phi^{r}\right|^{2}$, we conclude

$$
\begin{aligned}
& \int_{B_{2}} \int_{0}^{1} \frac{1}{\rho}|j-\rho \nabla \phi|^{2}-\left(\int_{B_{R}} \int_{0}^{1} \frac{1}{\rho}|j|^{2}-\int_{B_{R}}|\nabla \phi|^{2}\right) \\
& =\int_{B_{2}} \int_{0}^{1} \frac{1}{\rho}|j-\rho \nabla \phi|^{2}-\int_{B_{R}} \int_{0}^{1} \frac{1}{\rho}\left|j-\rho \nabla \phi^{r}\right|^{2} \\
& \quad+\int_{B_{R}}|\nabla \phi|^{2}-\int_{B_{R}}\left|\nabla \phi^{r}\right|^{2} \\
& \quad+\int_{B_{R}} \int_{0}^{1} \frac{1}{\rho}\left|j-\rho \nabla \phi^{r}\right|^{2}-\left(\int_{B_{R}} \int_{0}^{1} \frac{1}{\rho}|j|^{2}-\int_{B_{R}}\left|\nabla \phi^{r}\right|^{2}\right) \\
& \lesssim r(E+D)+r E+\frac{1}{r} D+\frac{1}{r^{d+2}} D^{2}+\frac{1}{r^{d+1}} E^{2}+\frac{1}{r^{\frac{d+1}{2}}} M^{\frac{1}{2}}(E+D)^{\frac{5}{4}} \\
& \lesssim r E+\frac{1}{r} D+\frac{1}{r^{d+2}} D^{2}+\frac{1}{r^{d+1}} E^{2}+\frac{1}{r^{\frac{d+1}{2}}} M^{\frac{1}{2}}(E+D)^{\frac{5}{4}} .
\end{aligned}
$$

Therefore, for every fixed $r>0$, provided $E+D$ is small enough we see that (3.57) holds. 


\subsubsection{The construction}

We now turn to the construction part of the proof i.e. Lemma 1.8,

Lemma 1.8. For every $0<\tau \ll 1$, there exist $\varepsilon(\tau)>0$ and $C(\tau)>0$ such that if $E+D \leq \varepsilon$, then there exists a density-flux pair $(\widetilde{\rho}, \widetilde{j})$ satisfying (3.13) and such that

$$
\iint_{0}^{1} \frac{1}{\widetilde{\rho}}|\widetilde{j}|^{2}-\int_{B_{R}}|\nabla \phi|^{2} \leq \tau E+C D
$$

Proof. We split the construction and its estimate into the following steps:

- SteP 1: the construction in the initial layer $B_{R} \times(0, \tau)$, using (3.51) to connect the measure $\mu^{\prime}$ defined in (3.36) to the constant $\kappa_{\mu^{\prime}}$ and in the final layer $B_{R} \times(1-\tau, 1)$, connecting in a similar way the constant $\kappa_{\lambda^{\prime}}$ to the measure $\lambda^{\prime}$ (see Figure 4),

- SteP 2: the construction in the boundary layer $\left(B_{R} \backslash \bar{B}_{R-r}\right) \times(\tau, 1-\tau)$ (see Figure 5), to connect the constant-in-t flux (cf. (3.30) $\hat{g}^{\prime}$ to (cf. (3.29) and (3.30)

$$
\widetilde{g}^{\prime}:= \begin{cases}\frac{1}{\tau} \hat{g}_{+}^{\prime} & \text { for } t \in(\tau, 2 \tau) \\ -\frac{1}{\tau} \hat{g}_{-}^{\prime} & \text { for } t \in(1-2 \tau, 1-\tau),\end{cases}
$$

- STEP 3: the leading order construction in $B_{R} \times(\tau, 1-\tau)$ to connect the constant $\kappa_{\mu^{\prime}}$ to the constant $\kappa_{\lambda^{\prime}}$ (see Figure 5), using $\nabla \phi^{\prime}$ defined by

$$
\Delta \phi^{\prime}=c \text { in } B_{R} \quad \text { and } \quad \nu \cdot \nabla \phi^{\prime}=\hat{g}^{\prime} \text { on } \partial B_{R},
$$

as flux,

- Step 4: the construction in the boundary $\partial B_{R} \times(\tau, 1-\tau)$, to connect the flux $\widetilde{g}^{\prime}$ to the flux $f^{\prime}$ defined in (3.28) (see Figure 6),

- Step 5: the estimate of cost of kept trajectories (see Figure 7),

- Step 6: the passage from $\phi^{\prime}$ to $\phi$,

- Step 7: the collection of the error terms.

Before starting, we notice for further reference that

$$
\int_{B_{R}}\left(\left|\nabla \phi^{\prime}\right|^{2}+|\nabla \phi|^{2}\right) \stackrel{\sqrt[2.11]{\lesssim}}{\lesssim} \int_{\partial B_{R}}\left(\left(\hat{g}^{\prime}\right)^{2}+\hat{g}^{2}\right) \stackrel{\sqrt[3.48]{\&} \underset{3.47}{\lesssim}}{\lesssim} E+D .
$$

Step 1. Construction in the initial and final layers. By the Eulerian formulation of the Wasserstein distance (cf. (3.51)), there exists $\left(\rho^{\text {in }}, j^{\text {in }}\right)$ supported in $\bar{B}_{R} \times[0, \tau]$ such that (see Figure 4 )

$$
\int_{B_{R}} \int_{0}^{1} \partial_{t} \zeta d \rho^{\text {in }}+\nabla \zeta \cdot d j^{\text {in }}=\int_{B_{R}}\left(\zeta_{\tau} \kappa_{\mu^{\prime}} d x-\zeta_{0} d \mu^{\prime}\right)
$$

and

$$
\int_{B_{R}} \int_{0}^{1} \frac{1}{\rho^{\text {in }}}\left|j^{\text {in }}\right|^{2}=\frac{1}{\tau} W_{B_{R}}^{2}\left(\mu^{\prime}, \kappa_{\mu^{\prime}}\right) \stackrel{\sqrt[3.51]{\lesssim}}{\lesssim} \tau E+\frac{1}{\tau} D
$$




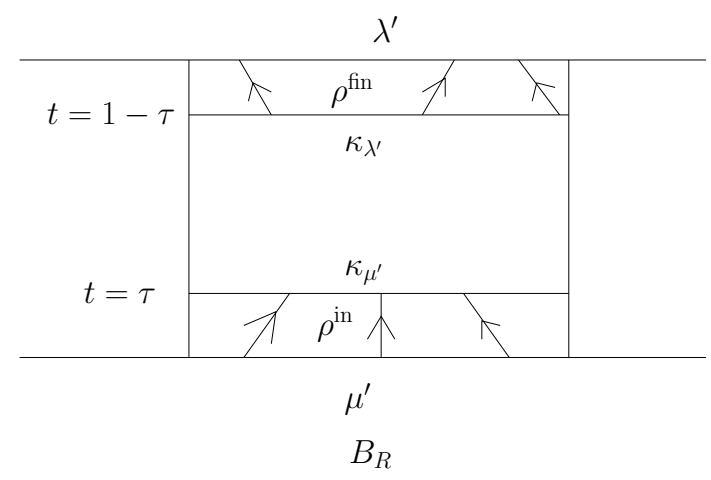

Figure 4: Initial and final layers.

Similarly, there exists $\left(\rho^{\mathrm{fin}}, j^{\mathrm{fin}}\right)$ supported in $B_{R} \times[1-\tau, 1]$ such that (see Figure 4 )

$$
\int_{B_{R}} \int_{0}^{1} \partial_{t} \zeta d \rho^{\mathrm{fin}}+\nabla \zeta \cdot d j^{\mathrm{fin}}=\int_{B_{R}}\left(\zeta_{1} d \lambda^{\prime}-\zeta_{1-\tau} \kappa_{\lambda^{\prime}} d x\right)
$$

and

$$
\int_{B_{R}} \int_{0}^{1} \frac{1}{\rho^{\text {fin }}}\left|j^{\text {fin }}\right|^{2} \lesssim \tau E+\frac{1}{\tau} D
$$

Step 2. Construction in the boundary layer. By definition (3.61) of $\widetilde{g}^{\prime}$, we have for every $x \in \partial B_{R}$,

$$
\int_{\tau}^{1-\tau} \widetilde{g}^{\prime}(t, x) d t=\int_{\tau}^{1-\tau} \frac{1}{1-2 \tau} \hat{g}^{\prime}(x) d t
$$

and

$$
\int_{\partial B_{R}} \int_{\tau}^{1-\tau}\left(\widetilde{g}^{\prime}-\frac{1}{1-2 \tau} \hat{g}^{\prime}\right)^{2} \lesssim \frac{1}{\tau} \int_{\partial B_{R}}\left(\hat{g}^{\prime}\right)^{2} \stackrel{\sqrt{3.48}}{\lesssim} \frac{1}{\tau}(E+D) .
$$

Therefore, applying [26, Lem. 2.4] to $\widetilde{g}^{\prime}-\frac{1}{1-2 \tau} \hat{g}^{\prime}$ and choosing $r$ to be a large but order one multiple of $\left(\int_{B_{R}} \int_{\tau}^{1-\tau}\left(\widetilde{g}^{\prime}-\frac{1}{1-2 \tau} \hat{g}^{\prime}\right)^{2}\right)^{\frac{1}{d+1}}$, we get the existence of a pair $(s, q)$ supported in $B_{R} \backslash \bar{B}_{R-r} \times(\tau, 1-\tau)$ (see Figure 5) with $|s| \leq \frac{1}{2}$, such that

$$
\int_{B_{R}} \int_{\tau}^{1-\tau} \partial_{t} \zeta d s+\nabla \zeta \cdot d q=\int_{\partial B_{R}} \int_{\tau}^{1-\tau} \zeta\left(\widetilde{g}^{\prime}-\frac{1}{1-2 \tau} \hat{g}^{\prime}\right)
$$

and

$$
\int_{B_{R} \backslash B_{R-r}} \int_{\tau}^{1-\tau}|q|^{2} \lesssim r \int_{\partial B_{R}} \int_{\tau}^{1-\tau}\left(\widetilde{g}^{\prime}-\frac{1}{1-2 \tau} \hat{g}^{\prime}\right)^{2} \lesssim\left(\frac{1}{\tau}(E+D)\right)^{\frac{d+2}{d+1}}
$$

Step 3. Leading order construction. For $t \in[\tau, 1-\tau]$ we define (see Figure 5) 


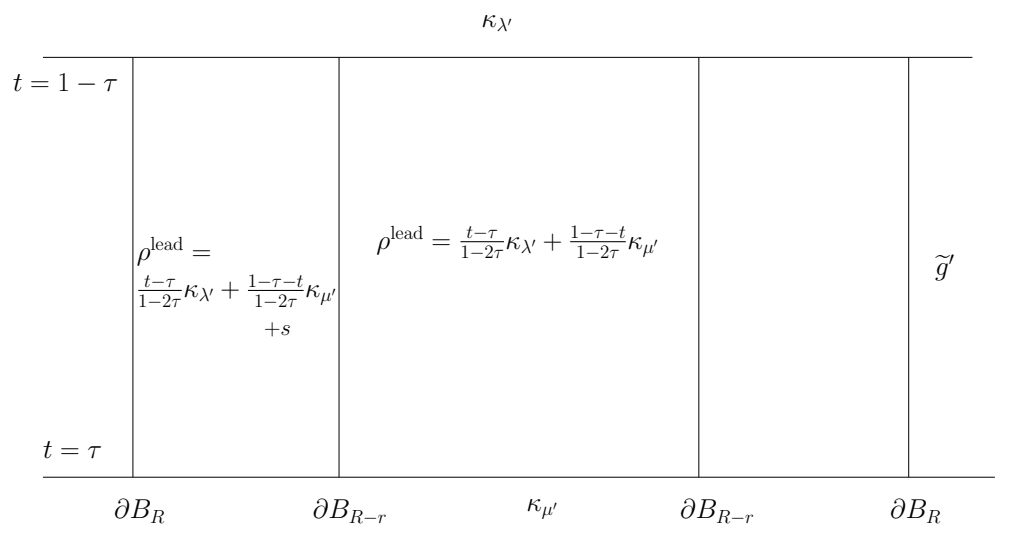

Figure 5: Leading order construction.

$$
\rho^{\text {lead }}:=\frac{t-\tau}{1-2 \tau} \kappa_{\lambda^{\prime}}+\frac{1-\tau-t}{1-2 \tau} \kappa_{\mu^{\prime}}+s, \quad j^{\text {lead }}:=\frac{1}{1-2 \tau} \nabla \phi^{\prime}+q,
$$

both restricted to $B_{R} \times[\tau, 1-\tau]$. Notice that by (3.35) and (3.27), $\Omega_{0} \backslash \Omega_{1}=\Omega_{+}^{\prime} \backslash \Omega_{-}^{\prime}=$ $\left\{|X(0)|<R,|X(1)| \geq R, t_{+} \geq 3 \tau\right\}$ and likewise $\Omega_{1} \backslash \Omega_{0}=\Omega_{-}^{\prime} \backslash \Omega_{+}^{\prime}$, and therefore

$$
\begin{aligned}
\left|B_{R}\right|\left(\kappa_{\mu^{\prime}}-\kappa_{\lambda^{\prime}}\right)=\int_{B_{R}}\left(d \mu^{\prime}-d \lambda^{\prime}\right) \stackrel{\sqrt{3.36}}{-} & \mathbb{P}\left(\Omega_{0}\right)-\mathbb{P}\left(\Omega_{1}\right) \\
& =\mathbb{P}\left(\Omega_{+}^{\prime}\right)-\mathbb{P}\left(\Omega_{-}^{\prime}\right)^{\sqrt[3.29]{-}} \int_{\partial B_{R}}\left(\hat{g}_{+}^{\prime}-\hat{g}_{-}^{\prime}\right),
\end{aligned}
$$

so that using (3.68) and 3.62

$$
\int_{B_{R}} \int_{\tau}^{1-\tau} \partial_{t} \zeta d \rho^{\text {lead }}+\nabla \zeta \cdot d j^{\text {lead }}=\int_{B_{R}}\left(\zeta_{1-\tau} \kappa_{\lambda^{\prime}}-\zeta_{\tau} \kappa_{\mu^{\prime}}\right)+\int_{\partial B_{R}} \int_{\tau}^{1-\tau} \zeta \widetilde{g}^{\prime} .
$$

Using that $(s, q)$ is supported in $B_{R} \backslash \bar{B}_{R-r} \times(\tau, 1-\tau)$ with $|s| \leq \frac{1}{2}$, the cost is majorized by

$$
\begin{aligned}
\int_{B_{R}} \int_{\tau}^{1-\tau} \frac{1}{\rho^{\text {lead }}}\left|j^{\text {lead }}\right|^{2}-\frac{1}{1-2 \tau} \int_{B_{r}}\left|\nabla \phi^{\prime}\right|^{2} & \\
& \lesssim \int_{B_{R} \backslash B_{R-r}}\left|\nabla \phi^{\prime}\right|^{2}+\int_{\tau}^{1-\tau} \int_{B_{R} \backslash B_{R-r}}|q|^{2} .
\end{aligned}
$$

Therefore, using (3.63), 3.51), 2.6), and (3.69) and recalling the choice of $r$ (see Step 2) we find

$$
\begin{aligned}
\int_{B_{R}} \int_{\tau}^{1-\tau} & \frac{1}{\rho^{\text {lead }}}\left|j^{\text {lead }}\right|^{2}-\int_{B_{R}}\left|\nabla \phi^{\prime}\right|^{2} \\
\lesssim\left(\tau+\left(\tau^{2} E+D\right)^{\frac{1}{2}}\right)(E+D)+ & r(E+D)+\left(\frac{1}{\tau}(E+D)\right)^{\frac{d+2}{d+1}} \\
& \lesssim \tau E+\frac{1}{\tau} D+\left(\frac{1}{\tau}(E+D)\right)^{\frac{d+2}{d+1}}
\end{aligned}
$$



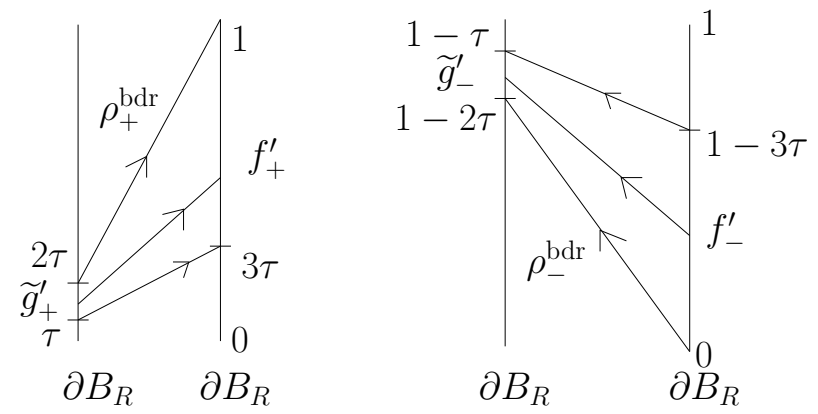

Figure 6: Construction in the boundary.

Step 4. Construction in the Boundary. We will separate the construction for the terms with the +-sign and with the --sign. We start with the +-sign. Notice that by definition (3.61), the positive part of $\widetilde{g}^{\prime}$, denoted by $\widetilde{g}_{+}^{\prime}$, satisfies $\int_{0}^{1} \widetilde{g}_{+}^{\prime}=\hat{g}_{+}^{\prime}$ and Spt $\widetilde{g}_{+}^{\prime} \subset \partial B_{R} \times(\tau, 2 \tau)$. By definition (3.28), we have $\int_{0}^{1} f_{+}^{\prime}=\bar{f}_{+}^{\prime}$ and Spt $f_{+}^{\prime} \subset$ $\partial B_{R} \times(3 \tau, 1)$. Hence, we may apply Lemma 2.6 to $f=\widetilde{g}_{+}^{\prime}$ and $g=f_{+}^{\prime}$ to obtain a density-flux pair $\left(\rho_{+}^{\mathrm{bdr}}, j_{+}^{\mathrm{bdr}}\right)$ in $\partial B_{R} \times(0,1)$ with (see Figure 6 )

$$
\int_{\partial B_{R}} \int_{0}^{1} \partial_{t} \zeta d \rho_{+}^{\mathrm{bdr}}+\nabla \zeta \cdot d j_{+}^{\mathrm{bdr}}=\int_{\partial B_{R}} \int_{0}^{1} \zeta d\left(f_{+}^{\prime}-\widetilde{g}_{+}^{\prime}\right)
$$

and

$$
\int_{\partial B_{R}} \int_{0}^{1} \frac{1}{\rho_{+}^{\mathrm{bdr}}}\left|j_{+}^{\mathrm{bdr}}\right|^{2} \leq \frac{1}{\tau} W_{\partial B_{R}}^{2}\left(\hat{g}_{+}^{\prime} \bar{f}_{+}^{\prime}\right) \stackrel{(3.49)}{\lesssim} \frac{M}{\tau}(E+D) .
$$

Similarly, applying Lemma 2.6 to $f=f_{-}^{\prime}$ and $g=\widetilde{g}_{-}^{\prime}$, we obtain a density-flux pair $\left(\rho_{-}^{\mathrm{bdr}}, j_{-}^{\mathrm{bdr}}\right)$ in $\partial B_{R} \times(0,1)$ with (see Figure 6 )

$$
\int_{\partial B_{R}} \int_{0}^{1} \partial_{t} \zeta d \rho_{-}^{\mathrm{bdr}}+\nabla \zeta \cdot d j_{-}^{\mathrm{bdr}}=\int_{\partial B_{R}} \int_{0}^{1} \zeta d\left(\widetilde{g}_{-}^{\prime}-f_{-}^{\prime}\right)
$$

and

$$
\int_{\partial B_{R}} \int_{0}^{1} \frac{1}{\rho_{-}^{\mathrm{bdr}}}\left|j_{-}^{\mathrm{bdr}}\right|^{2} \lesssim \frac{M}{\tau}(E+D)
$$

Letting $\left(\rho^{\mathrm{bdr}}, j^{\mathrm{bdr}}\right):=\left(\rho_{+}^{\mathrm{bdr}}+\rho_{-}^{\mathrm{bdr}}, j_{+}^{\mathrm{bdr}}+j_{-}^{\mathrm{bdr}}\right)$, we conclude that

$$
\int_{\partial B_{R}} \int_{0}^{1} \partial_{t} \zeta d \rho^{\mathrm{bdr}}+\nabla \zeta \cdot d j^{\mathrm{bdr}}=\int_{\partial B_{R}} \int_{0}^{1} \zeta d\left(f^{\prime}-\tilde{g}^{\prime}\right)
$$

and

$$
\int_{\partial B_{R}} \int_{0}^{1} \frac{1}{\rho^{\mathrm{bdr}}}\left|j^{\mathrm{bdr}}\right|^{2} \lesssim \frac{M}{\tau}(E+D) .
$$

Step 5. Cost of KePt trajectories. Recalling the definition $(3.26)$ of $\delta \Omega$, we let for $t \in(0,1)$ in line with $(3.3)$ (see Figure 7), 


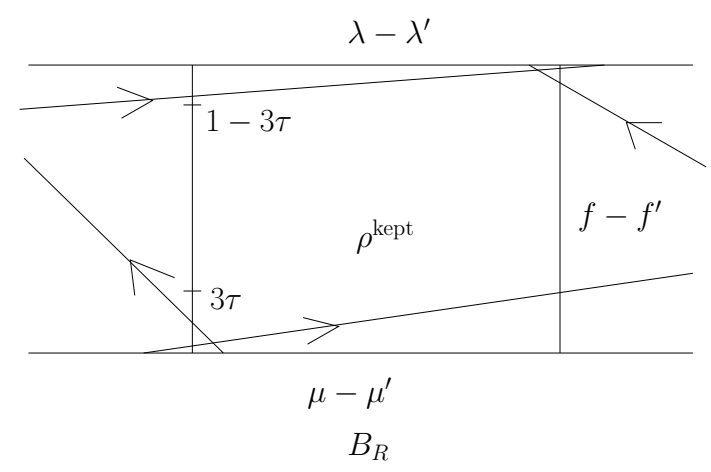

Figure 7: Kept trajectories.

$$
\begin{aligned}
\int \zeta d \rho_{t}^{\mathrm{kept}} & =\int_{\delta \Omega} I(|X(t)|<R) \zeta(X(t)) d \mathbb{P}, \\
\int \xi \cdot d j_{t}^{\mathrm{kept}} & =\int_{\delta \Omega} I(|X(t)|<R) \xi(X(t)) \cdot \dot{X}(t) d \mathbb{P}
\end{aligned}
$$

so that by definition (3.28) of $f_{R}^{\prime}$ and 3.36 of $\mu^{\prime}$ and $\lambda^{\prime}$,

$$
\begin{aligned}
\int_{B_{R}} \int_{0}^{1} \partial_{t} \zeta d \rho^{\mathrm{kept}}+\nabla \zeta \cdot d j^{\mathrm{kept}} & \\
& =\int_{B_{R}} \zeta_{1} d\left(\lambda-\lambda^{\prime}\right)+\int_{B_{R}} \zeta_{0} d\left(\mu-\mu^{\prime}\right)+\int_{\partial B_{R}} \int_{0}^{1} \zeta d\left(f-f^{\prime}\right) .
\end{aligned}
$$

By the representation 2.20 , we may bound

$$
\begin{aligned}
\frac{1}{2} \int_{B_{R}} \int_{0}^{1} \frac{1}{\rho^{\mathrm{kept}}}\left|j^{\mathrm{kept}}\right|^{2}=\sup _{\xi \in C_{c}^{0}\left(B_{R} \times[0,1]\right)^{d}} \int \xi \cdot d j-\frac{|\xi|^{2}}{2} d \rho & \\
=\sup _{\xi \in C_{c}^{0}\left(B_{R} \times[0,1]\right)^{d}} \int_{\delta \Omega} \int_{0}^{1} I(|X(t)| & <R)\left(\xi \cdot \dot{X}-\frac{|\xi|^{2}}{2}\right) d t d \mathbb{P} \\
& \leq \frac{1}{2} \int_{\delta \Omega} \int_{t_{-}}^{t_{+}}|\dot{X}|^{2} d t d \mathbb{P} \lesssim \tau E
\end{aligned}
$$

where in the last estimate we used that trajectories are straight and that for $X \in \delta \Omega$, $\left|t_{+}-t_{-}\right| \leq 3 \tau$.

Step 6. The passage from $\phi^{\prime}$ To $\phi$. Since $\int_{B_{R}} \phi=\int_{B_{R}} \phi^{\prime}=0$, using the definitions (3.53), 3.62 and integration by parts, we obtain

$$
\int_{B_{R}}\left|\nabla \phi^{\prime}\right|^{2}-\int_{B_{R}}|\nabla \phi|^{2}=\int_{\partial B_{R}}\left(\phi^{\prime}+\phi\right)\left(\hat{g}^{\prime}-\hat{g}\right) .
$$


Therefore, by Cauchy-Schwarz and the Poincaré-trace estimate,

$$
\begin{aligned}
\left.\left|\int_{B_{R}}\right| \nabla \phi^{\prime}\right|^{2}-\int_{B_{R}}|\nabla \phi|^{2} \mid & \lesssim\left(\left(\int_{B_{R}}\left|\nabla \phi^{\prime}\right|^{2}+\int_{B_{R}}|\nabla \phi|^{2}\right) \int_{\partial B_{R}}\left(\hat{g}^{\prime}-\hat{g}\right)^{2}\right)^{\frac{1}{2}} \\
& \stackrel{3.63}{\lesssim}\left((E+D) \int_{\partial B_{R}}\left(\hat{g}_{ \pm}^{\prime}-\hat{g}_{ \pm}\right)^{2}\right)^{\frac{1}{2}} \\
& \stackrel{3.50}{\lesssim}\left((E+D)\left(\tau^{2} E+D\right)\right)^{\frac{1}{2}} \lesssim \tau E+\frac{1}{\tau} D .
\end{aligned}
$$

Step 7. Collecting ERror terms. We use

$$
(\widetilde{\rho}, \widetilde{j}):=\left(\rho^{\mathrm{in}}+\rho^{\mathrm{fin}}+\rho^{\text {lead }}+\rho^{\mathrm{bdr}}+\rho^{\mathrm{kept}}, j^{\text {in }}+j^{\text {fin }}+j^{\text {lead }}+j^{\mathrm{bdr}}+j^{\text {kept }}\right)
$$

as our competitor. Putting together (3.64), (3.66), (3.71), 3.73) and (3.75), we see that the continuity equation (3.13) is satisfied. Putting together estimates (3.65) and (3.67) coming from the construction in the final and initial layers, 3.72$)$ coming from the leading order construction, (3.74) coming from transporting in $\partial B_{R}$, 3.76) counting the contribution from the kept trajectories and (3.77) estimating the error of passing from $\phi^{\prime}$ to $\phi$, we get

$$
\begin{aligned}
& \int_{B_{R}} \int_{0}^{1} \frac{1}{\widetilde{\rho}}|\widetilde{j}|^{2}-\int_{B_{R}}|\nabla \phi|^{2} \\
& \lesssim \tau E+\frac{1}{\tau} D+\left(\frac{1}{\tau}(E+D)\right)^{\frac{d+2}{d+1}}+\frac{M}{\tau}(E+D)+\tau E+\frac{1}{\tau} D \\
& \lesssim\left(\tau+\frac{M}{\tau}\right) E+\left(\frac{1}{\tau}(E+D)\right)^{\frac{d+2}{d+1}}+\frac{1}{\tau} D,
\end{aligned}
$$

from which 3.60 follows if $E+D$ is small enough.

\subsection{Proof of the harmonic approximation result}

We may now finally prove our main harmonic approximation result in its Lagrangian version, Theorem 1.4

Theorem 1.4. For every $\tau>0$, there exist positive constants $\varepsilon(\tau)$ and $C(\tau)$ such that if $\pi$ is an optimal transference plan between two measures $\mu$ and $\lambda$ with $E+D \leq \varepsilon$, then there exists a radius $R \in(3,4)$ such that if $\Phi$ is given by

$$
\Delta \Phi=c \text { in } B_{R} \quad \text { and } \quad \nu \cdot \nabla \Phi=\nu \cdot \bar{j} \text { on } \partial B_{R},
$$

then

$$
\int_{\left(B_{1} \times \mathbb{R}^{d}\right) \cup\left(\mathbb{R}^{d} \times B_{1}\right)}|x-y+\nabla \Phi(x)|^{2} d \pi \leq \tau E+C D
$$

and

$$
\sup _{B_{2}}\left|\nabla^{2} \Phi\right|^{2}+\sup _{B_{2}}|\nabla \Phi|^{2} \lesssim E+D
$$


Proof. The proof is almost identical to the proof of [26, Prop. 3.6] but we give it for the reader's convenience. Let us first notice that $(3.79)$ is given by $(3.55)$. We thus only need to derive (3.78) from (3.54). Since $\nabla \Phi$ is smooth in $B_{2}$, we obtain as for 2.22 , that

$\iint_{0}^{1} I(|t y+(1-t) x|<2)|x-y+\nabla \Phi(t y+(1-t) x)|^{2} d \pi d t=\int_{B_{2}} \int_{0}^{1} \frac{1}{\rho}|j-\rho \nabla \Phi|^{2}$.

Recalling that by 2.35) of Lemma 2.9, if $x \in B_{1}$ or $y \in B_{1}$ and $(x, y) \in \operatorname{Spt} \pi$ then $t y+(1-t) x \in B_{2}$, we may therefore estimate

$$
\begin{aligned}
& \int_{\left(B_{1} \times \mathbb{R}^{d}\right) \cup\left(\mathbb{R}^{d} \times B_{1}\right)}|x-y+\nabla \Phi(x)|^{2} d \pi \\
& \lesssim \int_{\left(B_{1} \times \mathbb{R}^{d}\right) \cup\left(\mathbb{R}^{d} \times B_{1}\right)} \int_{0}^{1}|x-y+\nabla \Phi(t y+(1-t) x)|^{2} d \pi d t \\
& \quad+\int_{\left(B_{1} \times \mathbb{R}^{d}\right) \cup\left(\mathbb{R}^{d} \times B_{1}\right)} \int_{0}^{1}|\nabla \Phi(x)-\nabla \Phi(t y+(1-t) x)|^{2} d \pi d t \\
& \lesssim \int_{B_{2}} \int_{0}^{1} \frac{1}{\rho}|j-\rho \nabla \Phi|^{2}+\sup _{B_{2}}\left|\nabla^{2} \Phi\right|^{2} \int_{\left(B_{1} \times \mathbb{R}^{d}\right) \cup\left(\mathbb{R}^{d} \times B_{1}\right)}|y-x|^{2} d \pi \\
& \underset{\Sigma}{\lesssim} \tau E+C(\tau) D+(E+D) E .
\end{aligned}
$$

This concludes the proof of $(3.78)$, provided $E+D$ is small enough.

\section{Quantitative bounds on the displacement}

In this section we prove Theorem 1.2 . For a given measure $\pi$ on $\mathbb{R}^{d} \times \mathbb{R}^{d}$ with cyclically monotone support and $\bar{R}>0$, we let $\mu:=\pi_{1}\left\llcorner B_{\bar{R}}\right.$ and assume from now on that $\pi_{2}\left\llcorner B_{\bar{R}}=d x\right.$.

Let us recall that we fixed a nonnegative rate function $\beta$ satisfying the following hypothesis (see (1.7)): it is increasing, $R \mapsto \frac{\beta(R)}{R}$ is decreasing, and there exists a constant $C_{\beta}>0$ such that

$$
\sum_{\ell \geq 0} \frac{\beta\left(2^{\ell} R\right)}{2^{\ell} R} \leq C_{\beta} \frac{\beta(R)}{R} \quad \text { for every } R \geq 1 \quad \text { and } \quad \beta(1) \leq C_{\beta} .
$$

Note that this in particular implies for any $\ell \in \mathbb{N}$ and $R>2^{\ell}$

$$
2^{-\ell} \beta(R) \lesssim \beta\left(2^{-\ell} R\right)
$$

Moreover, since $R \mapsto \frac{\beta(R)}{R}$ is decreasing, for $R \gg 1$, there holds

$$
\beta(R) \ll R^{2} .
$$




\subsection{The Campanato iteration}

We first prove that as a consequence of Theorem 1.4 and a Campanato iteration, we can obtain an $L^{2}$ bound on the displacement, that is Proposition 1.9 , which we now restate. To this aim, let us first recall some notation. For a sequence of approximately geometric radii i.e.

$$
\bar{R} \geq R_{0} \geq \cdots \geq R_{K} \geq C \quad \text { with } \quad R_{k-1} \geq 2 R_{k} \geq \frac{1}{C} R_{k-1}
$$

with $\bar{R} \sim R_{0}$ and $R_{K} \sim 1$, we put

$$
E_{k}=\frac{1}{\left|B_{6 R_{k}}\right|} \int_{\left(B_{6 R_{k}} \times \mathbb{R}^{d}\right) \cup\left(\mathbb{R}^{d} \times B_{6 R_{k}}\right)}|x-y|^{2} d \pi_{k}
$$

and consider $\pi_{k}$ the coupling defined recursively by $\pi_{0}:=\pi$ and

$$
\pi_{k}=\left(\mathrm{id}, \mathrm{id}-\nabla \Phi_{k-1}(0)\right) \# \pi_{k-1},
$$

where $\Phi_{k}$ solves the Poisson equation

$$
\Delta \Phi_{k}=c \text { in } B_{R_{k}} \quad \text { and } \quad \nu \cdot \nabla \Phi_{k}=\nu \cdot \bar{j}_{k} \text { on } \partial B_{R_{k}} .
$$

As in 2.21, the flux $j_{k}$ is the Eulerian flux related to $\pi_{k}$, that is

$$
\int \xi \cdot j_{k, t}=\int \xi((1-t) x+t y) \cdot(y-x) d \pi_{k}
$$

from which $\bar{j}_{k}$ is obtained by integrating in time (see (2.1)). Let us also recall that from 2.24 and the invariance of the Lebesgue measure under translation,

$$
\nabla \cdot \bar{j}_{k}=\mu-1 \quad \text { in } B_{R_{k}} .
$$

Proposition 1.9. Assume that

$$
\frac{1}{\left|B_{\bar{R}}\right|} \int_{\left(B_{\bar{R}} \times \mathbb{R}^{d}\right) \cup\left(\mathbb{R}^{d} \times B_{\bar{R}}\right)}|x-y|^{2} d \pi \leq \beta(\bar{R})
$$

and

$$
\frac{1}{\left|B_{R}\right|} W_{B_{R}}^{2}(\mu, \kappa) \leq \beta(R) \quad \forall R \in[1, \bar{R}] .
$$

Then, there exists a sequence of approximately geometric radii $\left(R_{k}\right)_{0 \leq k \leq K}$ such that

$$
E_{k} \lesssim \beta\left(R_{k}\right)
$$

and

$$
\left|\nabla \Phi_{k}(0)\right|^{2} \lesssim \beta\left(R_{k}\right)
$$


Proof. Let $6 \bar{R}_{0}=\bar{R}$ and for $\theta \ll 1$ dyadic to be fixed later, let $6 \bar{R}_{k}:=\theta^{k} \bar{R}_{0}$. We prove by induction that there exists a constant $C_{E}>0$ only depending on $d$ and $\beta$ such that for $k \in[0, K]$, there exists $R_{k} \in\left(3 \bar{R}_{k}, 4 \bar{R}_{k}\right)$ such that defining $\Phi_{k-1}$ through (4.6) and then $\pi_{k}$ as in (4.5), we have that the support of $\pi_{k}$ is cyclically monotone, that the first marginal of $\pi_{k}$ in $B_{6} \bar{R}_{k}$ is $\mu$, that its second marginal in $B_{6} \bar{R}_{k}$ is the Lebesgue measure, and that both 4.12 and

$$
\bar{E}_{k}:=\frac{1}{\left|B_{6 \bar{R}_{k}}\right|} \int_{\left(B_{6 \bar{R}_{k}} \times \mathbb{R}^{d}\right) \cup\left(\mathbb{R}^{d} \times B_{6} \bar{R}_{k}\right)}|x-y|^{2} d \pi_{k} \leq C_{E} \beta\left(\bar{R}_{k}\right)
$$

hold. This would upgrade to (4.11). Indeed, by assumption (4.9), 4.11) holds for $k=0$ and if $k \geq 1$, we have by $(4.5)$,

$$
\begin{aligned}
& \frac{1}{\left|B_{6 R_{k}}\right|} \int_{\left(B_{6 R_{k}} \times \mathbb{R}^{d}\right) \cup\left(\mathbb{R}^{d} \times B_{6 R_{k}}\right)}|x-y|^{2} d \pi_{k} \\
& =\frac{1}{\left|B_{6 R_{k}}\right|} \int_{\left(B_{6 R_{k}} \times \mathbb{R}^{d}\right) \cup\left(\mathbb{R}^{d} \times B_{6 R_{k}}\left(\nabla \Phi_{k-1}(0)\right)\right)}\left|x-y+\nabla \Phi_{k-1}(0)\right|^{2} d \pi_{k-1} \\
& \lesssim \frac{1}{\left|B_{6 R_{k}}\right|} \int_{\left(B_{7 R_{k}} \times \mathbb{R}^{d}\right) \cup\left(\mathbb{R}^{d} \times B_{7 R_{k}}\right)}|x-y|^{2} d \pi_{k-1}+\left|\nabla \Phi_{k-1}(0)\right|^{2},
\end{aligned}
$$

where we used that by 4.12 and 4.3$) B_{6 R_{k}}\left(\nabla \Phi_{k-1}(0)\right) \subset B_{7 R_{k}}$ and that by the $L^{\infty}$ bound (2.33) which we may apply since $E_{k-1} \leq \beta\left(R_{k-1}\right) \ll R_{k-1}^{2} \sim R_{k}^{2}$, Spt $\pi_{k-1} \cap$ $\left(B_{7 R_{k}} \times \mathbb{R}^{d}\right) \cup\left(\mathbb{R}^{d} \times B_{7 R_{k}}\right) \subset \mathbb{R}^{d} \times B_{9 R_{k}}$. Therefore, if $\theta$ is sufficiently small such that $7 R_{k} \leq 6 \bar{R}_{k-1}$, using (4.13) and (4.12), we would conclude the proof of (4.11).

We now turn to the inductive argument. Let $0<\tau \ll 1$ be such that Theorem 1.4 applies and define for $k$ such that $6 \bar{R}_{k} \geq 1$ (recall Remark 1.5 )

$$
\bar{D}_{k}:=\frac{1}{\left|B_{6 \bar{R}_{k}}\right|} W_{B_{6 \bar{R}_{k}}}^{2}(\mu, \kappa) \stackrel{4.9 p}{\leq} \beta\left(6 \bar{R}_{k}\right) \leq 6 \beta\left(\bar{R}_{k}\right) .
$$

By hypothesis (4.9), 4.11) holds for $k=0$ and $\frac{1}{\bar{R}_{0}^{2}} \bar{E}_{0}+\frac{1}{\bar{R}_{0}^{2}} \bar{D}_{0} \lesssim \frac{\beta\left(\bar{R}_{0}\right)}{\bar{R}_{0}^{2}} \ll 1$ so that by Theorem 1.4 , there exists $R_{0} \in\left(3 \bar{R}_{0}, 4 \bar{R}_{0}\right)$ such that defining $\Phi_{0}$ through (4.6), 4.12 holds for $k=0$.

Assume now that (4.13) holds for $k$. By (4.13) and (4.14), we have in particular $\frac{1}{\bar{R}_{k}^{2}} \bar{E}_{k}+\frac{1}{\bar{R}_{k}^{2}} \bar{D}_{k} \ll 1$ and thus by Theorem 1.4 . for any $0<\tau \ll 1$ there exists $R_{k} \in\left(3 \bar{R}_{k}, 4 \bar{R}_{k}\right)$ such that defining $\Phi_{k}$ through (4.6), we have (4.12) and

$$
\frac{1}{\left|B_{\bar{R}_{k}}\right|} \int_{\left(B_{\bar{R}_{k}} \times \mathbb{R}^{d}\right) \cup\left(\mathbb{R}^{d} \times B_{\bar{R}_{k}}\right)}\left|x-y+\nabla \Phi_{k}(x)\right|^{2} d \pi_{k} \leq \tau \bar{E}_{k}+C(\tau) \bar{D}_{k} .
$$

Defining $\pi_{k+1}$ through (4.5), we notice that if the support of $\pi_{k}$ is cyclically monotone, then also the support of $\pi_{k+1}$ is cyclically monotone. Moreover, using that by 4.12 ,

$$
B_{6 \bar{R}_{k+1}}\left(\nabla \Phi_{k}(0)\right)=B_{6 \theta \bar{R}_{k}}\left(\nabla \Phi_{k}(0)\right) \subset B_{7 \theta \bar{R}_{k}} \subset B_{\bar{R}_{k}}
$$


we still have that in $B_{6 \bar{R}_{k+1}}$, the first marginal of $\pi_{k+1}$ is $\mu$ and the second marginal is the Lebesgue measure. Moreover, by the $L^{\infty}$ bound 2.33 , ,

$$
\text { Spt } \pi_{k} \cap\left[\left(B_{6 \bar{R}_{k+1}} \times \mathbb{R}^{d}\right) \cup\left(\mathbb{R}^{d} \times B_{6 \bar{R}_{k+1}}\left(\nabla \Phi_{k}(0)\right)\right)\right] \subset B_{7 \theta \bar{R}_{k}} \times \mathbb{R}^{d},
$$

and we may compute

$$
\begin{aligned}
& \frac{1}{\left|B_{6 \bar{R}_{k+1}}\right|} \int_{\left(B_{6 \bar{R}_{k+1}} \times \mathbb{R}^{d}\right) \cup\left(\mathbb{R}^{d} \times B_{6 \bar{R}_{k+1}}\right)}|x-y|^{2} d \pi_{k+1} \\
& =\frac{1}{\left|B_{6 \theta \bar{R}_{k}}\right|} \int_{\left(B_{6 \theta \bar{R}_{k}} \times \mathbb{R}^{d}\right) \cup\left(\mathbb{R}^{d} \times B_{6 \theta \bar{R}_{k}}\left(\nabla \Phi_{k}(0)\right)\right)}\left|x-y+\nabla \Phi_{k}(0)\right|^{2} d \pi_{k} \\
& \stackrel{4.16] \& 4.17}{\lesssim} \frac{1}{\theta^{d}\left|B_{\bar{R}_{k}}\right|} \int_{\left(B_{\bar{R}_{k}} \times \mathbb{R}^{d}\right) \cup\left(\mathbb{R}^{d} \times B_{\bar{R}_{k}}\right)}\left|x-y+\nabla \Phi_{k}(x)\right|^{2} d \pi_{k} \\
& +\sup _{B_{7 \theta \bar{R}_{k}}}\left|\nabla \Phi_{k}-\nabla \Phi_{k}(0)\right|^{2} \\
& \underset{\theta^{4.15}}{\lesssim} \frac{\tau .79]}{\theta^{d}} \bar{E}_{k}+\frac{C(\tau)}{\theta^{d}} \bar{D}_{k}+\theta^{2}\left(\bar{E}_{k}+\bar{D}_{k}\right) \\
& \stackrel{4.133 \& 4.14}{\leq}\left(C C_{E}\left(\frac{\tau}{\theta^{d}}+\theta^{2}\right)+\theta C(\tau, \theta)\right) \beta\left(\bar{R}_{k}\right) \\
& \stackrel{4.2}{\leq}\left(C C_{E}\left(\frac{\tau}{\theta^{d+1}}+\theta\right)+C(\tau, \theta)\right) \beta\left(\bar{R}_{k+1}\right),
\end{aligned}
$$

where $C$ denotes a constant only depending on $d$ and $\beta$, but neither on $\tau$ nor on $\theta$. We first fix a dyadic $0<\theta \ll 1$ such that $C \theta \leq \frac{1}{4}$, then $0<\tau \ll 1$ such that $C \frac{\tau}{\theta^{d+1}} \leq \frac{1}{4}$ and finally let $C_{E}:=2 C(\tau, \theta)$, proving that 4.13 holds for $k+1$.

\subsection{Choosing good radii}

In this subsection, we show that without loss of generality we can make further restrictions on the sequence of radii $R_{k}$ given by Proposition 1.9. Let us recall that $\bar{R}_{k}=\theta^{k} \bar{R}_{0}$ and that for every $k \geq 0, R_{k} \in\left(3 \bar{R}_{k}, 4 \bar{R}_{k}\right)$ was chosen to be a good radius in the sense of Proposition 3.6. For notational simplicity we take as convention that

$$
\bar{j}_{-1}:=0 \quad \text { and } \quad E_{-1}:=\frac{1}{\left|B_{\bar{R}}\right|} \int_{\left(B_{\bar{R}} \times \mathbb{R}^{d}\right) \cup\left(\mathbb{R}^{d} \times B_{\bar{R}}\right)}|x-y|^{2} d \pi .
$$

Lemma 4.1. For every $k \in[0, K]$, we may assume that $R_{k}$ is both a good radius in the sense of Proposition 3.6, that $\mu\left(\partial B_{R_{k}}\right)=\left|\bar{j}_{k-1}\right|\left(\partial B_{R_{k}}\right)=0$ and

$$
\begin{gathered}
\frac{R_{k}}{\left|B_{R_{k}}\right|} \int_{0}^{1} \int\left|I\left(|(1-t) x+t y|<R_{k}\right)-I\left(|y|<R_{k}\right)\right| d \pi_{k} d t \lesssim \sqrt{E_{k-1}}, \\
\frac{R_{k}}{\left|B_{R_{k}}\right|} \int_{0}^{1} \int\left|I\left(|(1-t) x+t y|<R_{k}\right)-I\left(|y|<R_{k}\right)\right||x-y| d \pi_{k} d t \lesssim E_{k-1},
\end{gathered}
$$


hold. Moreover, setting $b_{k}:=\nabla \Phi_{k}(0)$, we may also assume that

$$
\begin{array}{r}
\frac{R_{k+1}}{\left|B_{R_{k+1}}\right|} \int_{0}^{1} \int\left|I\left((1-t) x+t y \mid<R_{k+1}\right)-I\left(\left|(1-t) x+t\left(y+b_{k}\right)\right|<R_{k+1}\right)\right| \\
|x-y| d \pi_{k+1} d t \lesssim\left|b_{k}\right| \sqrt{E_{k-1}} .
\end{array}
$$

holds.

Let us comment on Lemma 4.1 before proving it. We require here that the transport across $\partial B_{R_{k}}$ is not above average in two different ways: first on the level of the mass in (4.19) and second at the level of the displacement in (4.20). As a consequence, we prove in $(4.26)$ that on the level of the Eulerian fluxes, the Lagrangian shift $\nabla \Phi_{k}(0)$ has an almost additive effect. In (4.21), we further require that when choosing $R_{k+1}$ (after having chosen $R_{k}$ ), the displacement induced by the shift $\nabla \Phi_{k}(0)$ is not above average near $\partial B_{R_{k+1}}$.

Proof of Lemma 4.1. Since for every fixed $k \in[0, K]$, there exists a set of measure of order $\bar{R}_{k}$ in $\left(3 \bar{R}_{k}, 4 \bar{R}_{k}\right)$ of good radii in the sense of Proposition 3.6 , we may find $R_{k}$ which also satisfies $\mu\left(\partial B_{R_{k}}\right)=\left|\bar{j}_{k-1}\right|\left(\partial B_{R_{k}}\right)=0$, 4.19), 4.20) and (4.21) provided we show that

$$
\begin{array}{r}
\frac{1}{\left|B_{6 \bar{R}_{k}}\right|} \int_{3 \bar{R}_{k}}^{4 \bar{R}_{k}} \int_{0}^{1} \int|I(|(1-t) x+t y|<R)-I(|y|<R)| d \pi_{k} d t d R \lesssim \sqrt{E_{k-1}}, \\
\frac{1}{\left|B_{6 \bar{R}_{k}}\right|} \int_{3 \bar{R}_{k}}^{4 \bar{R}_{k}} \int_{0}^{1} \int|I(|(1-t) x+t y|<R)-I(|y|<R)| \\
|x-y| d \pi_{k} d t d R \leq E_{k-1}, \\
\frac{1}{\left|B_{6 \bar{R}_{k+1}}\right|} \int_{3 \bar{R}_{k+1}}^{4 \bar{R}_{k+1}} \int_{0}^{1} \int\left|I((1-t) x+t y \mid<R)-I\left(\left|(1-t) x+t\left(y+b_{k}\right)\right|<R\right)\right| \\
|x-y| d \pi_{k+1} d t d R \lesssim\left|b_{k}\right| \sqrt{E_{k-1}} .
\end{array}
$$

STEP 1. Let us start with the proof of 4.22. Using an exchange of integrals,

$$
\int_{0}^{\infty}|I(|(1-t) x+t y|<R)-I(|y|<R)| d R=||(1-t) x+t y|-| y|| \leq(1-t)|y-x|,
$$

and the $L^{\infty}$ bound 2.34 to reduce the integral to $\left(B_{6 \bar{R}_{k}} \times \mathbb{R}^{d}\right) \cup\left(\mathbb{R}^{d} \times B_{6} \bar{R}_{k}\right)$, we obtain

$$
\begin{aligned}
\int_{3 \bar{R}_{k}}^{4 \bar{R}_{k}} \int_{0}^{1} \int \mid I(|(1-t) x+t y|<R)-I(|y| & <R) \mid d \pi_{k} d t d R \\
& \leq \int_{\left(B_{6} \bar{R}_{k} \times \mathbb{R}^{d}\right) \cup\left(\mathbb{R}^{d} \times B_{6 \bar{R}_{k}}\right)}|x-y| d \pi_{k} .
\end{aligned}
$$


The desired estimate 4.22 follows since by Cauchy-Schwarz's inequality and the mass bound (2.36)

$$
\begin{aligned}
& \int_{\left(B_{6} \bar{R}_{k} \times \mathbb{R}^{d}\right) \cup\left(\mathbb{R}^{d} \times B_{6 \bar{R}_{k}}\right)}|x-y| d \pi_{k} \\
& \lesssim\left(\int_{\left(B_{6 \bar{R}_{k}} \times \mathbb{R}^{d}\right) \cup\left(\mathbb{R}^{d} \times B_{\left.6 \bar{R}_{k}\right)}\right.} d \pi_{k}\right)^{\frac{1}{2}}\left(\int_{\left(B_{6 \bar{R}_{k}} \times \mathbb{R}^{d}\right) \cup\left(\mathbb{R}^{d} \times B_{6 \bar{R}_{k}}\right)}|x-y|^{2} d \pi_{k}\right)^{\frac{1}{2}} \\
& \lesssim \sqrt{\bar{E}_{k}} \cdot\left|B_{6 \bar{R}_{k}}\right| \lesssim \sqrt{E_{k-1}} \cdot\left|B_{6 \bar{R}_{k}}\right|,
\end{aligned}
$$

where for the last inequality, one can argue as for the proof of (4.11).

Step 2. Estimate 4.23 is obtained arguing as for 4.22.

Step 3. We now turn to the proof of (4.24). On the one hand, this relies on the elementary

$$
\begin{array}{r}
\int_{0}^{\infty}\left|I((1-t) x+t y \mid<R)-I\left(\left|(1-t) x+t\left(y+b_{k}\right)\right|<R\right)\right| d R \\
=||(1-t) x+t\left(y+b_{k}\right)|-|(1-t) x+t y|| \leq t\left|b_{k}\right|,
\end{array}
$$

and on the other hand, on the fact that for fixed $t \in[0,1]$ and $R \in\left(3 \bar{R}_{k}, 4 \bar{R}_{k}\right)$, the statement $\left(|(1-t) x+t y|<R\right.$ or $\left.\left|(1-t) x+t\left(y+b_{k}\right)\right|<R\right)$ implies $|(1-t) x+t y|<$ $R+o(R)$, since by $(4.12)\left|b_{k}\right|^{2} \lesssim \beta(R) \ll R^{2}$, which in turn implies the statement $\left(|x|<6 \bar{R}_{k}\right.$ or $\left.|y|<6 \bar{R}_{k}\right)$ by (2.34). Then, we can argue as for 4.22).

We may derive Lemma 1.12 (which we now restate) from Lemma 4.1

Lemma 1.12. For every $k \in[1, K]$,

$$
\left|\frac{1}{\left|B_{R_{k}}\right|} \int_{B_{R_{k}}}\left(\bar{j}_{k}+\nabla \Phi_{k-1}(0)-\bar{j}_{k-1}\right)\right| \lesssim \frac{\beta\left(R_{k}\right)}{R_{k}} .
$$

Proof. Recall from Lemma 4.1 the abbreviation $b_{k-1}=\nabla \Phi_{k-1}(0)$. Using the definition (4.7) of $j_{k-1}$, the fact that the second marginal of $\pi_{k-1}$ is the Lebesgue measure, (4.2), 4.11), and 4.12), it is enough to prove that (recall 4.18)

$$
\begin{aligned}
& \mid \int_{0}^{1}\left(\int \left(I\left(|(1-t) x+t y|<R_{k}\right)(y-x) d \pi_{k}\right.\right. \\
& \left.\quad+\int\left(I\left(|y|<R_{k}\right) b_{k-1}-I\left(|(1-t) x+t y|<R_{k}\right)(y-x)\right) d \pi_{k-1}\right) d t \mid \\
& \quad \lesssim\left|b_{k-1}\right| \sqrt{E_{k-2}}\left|B_{R_{k}}\right| .
\end{aligned}
$$

By the recursive relation 4.5 this may be rearranged to

$$
\begin{aligned}
& \mid \int_{0}^{1}\left(\int\left(I\left(|(1-t) x+t y|<R_{k}\right)-I\left(\left|(1-t) x+t\left(y+b_{k}\right)\right|<R_{k}\right)\right)(y-x) d \pi_{k}\right. \\
& \left.\quad-\int\left(I\left(|(1-t) x+t y|<R_{k}\right)-I\left(|y|<R_{k}\right)\right) b_{k-1} d \pi_{k-1}\right) d t \mid \\
& \quad \lesssim\left|b_{k-1}\right| \sqrt{E_{k-2}}\left|B_{R_{k}}\right| .
\end{aligned}
$$


This is obtained using (4.21) for the first contribution and (4.19) for the second contribution.

\subsection{Flux of solutions to Poisson equations on concentric annuli}

For $R>0$ and $\mu$ a measure on $\mathbb{R}^{d}$, let $v^{R}$ be defined (up to an additive constant) through

$$
\Delta v^{R}=\mu-c \text { in } B_{R} \quad \text { and } \quad \nu \cdot \nabla v^{R}=0 \text { on } \partial B_{R} .
$$

The aim of this section is to prove the following summation formula (which is a restatement of Lemma 1.14):

Lemma 1.14. Let $R_{0}>\cdots>R_{k}$ be a sequence of decreasing radii with $\mu\left(\partial B_{R_{\ell}}\right)=0$ for every $\ell \in[1, k]$, then

$$
\frac{1}{\left|B_{R_{k}}\right|} \int_{\partial B_{R_{k}}} x \nu \cdot \nabla v^{R_{0}}=\sum_{\ell=1}^{k} \frac{1}{\left|B_{R_{\ell}}\right|} \int_{\partial B_{R_{\ell}}} x \nu \cdot \nabla v^{R_{\ell-1}} .
$$

This is obtained through a representation formula for $\frac{1}{\left|B_{r}\right|} \int_{\partial B_{r}} x \nu \cdot \nabla v^{R}$ for every $0<r<R$. Concentrating for instance on the first component, we have

Lemma 4.2. For $0<r<R$, let $\omega_{r}^{R}$ be the (piecewise smooth) solution of

$$
\begin{cases}\Delta \omega_{r}^{R}=0 & \text { in } B_{r} \cup\left(B_{R} \backslash \bar{B}_{r}\right), \\ {\left[\omega_{r}^{R}\right]=\frac{x_{1}}{\left|B_{r}\right|},\left[\nu \cdot \nabla \omega_{r}^{R}\right]=0} & \text { on } \partial B_{r}, \\ \nu \cdot \nabla \omega_{r}^{R}=0 & \text { on } \partial B_{R}, \\ \omega_{r}^{R}=0 & \text { in } B_{R}^{c}\end{cases}
$$

with [.] denoting the jump across $\partial B_{r}$ (outside minus inside); normalized by requiring

$$
\int_{B_{R}} \omega_{r}^{R}=0
$$

Then, for every $0<r<R$ with $\mu\left(\partial B_{r}\right)=\mu\left(\partial B_{R}\right)=0$, we have the representation formula

$$
\frac{1}{\left|B_{r}\right|} \int_{\partial B_{r}} x_{1} \nu \cdot \nabla v^{R}=-\int_{B_{R}} \omega_{r}^{R} d \mu .
$$

Moreover, for $R_{0}>\cdots>R_{k}$ a sequence of decreasing radii, we have the telescoping formula

$$
\omega_{R_{k}}^{R_{0}}=\sum_{\ell=1}^{k} \omega_{R_{\ell}}^{R_{\ell-1}}
$$


Finally, we have the explicit formula for $\nabla \omega_{r}^{R}$ :

$$
\begin{aligned}
& \nabla \omega_{r}^{R}(x) \\
& =\left\{\begin{array}{llr}
\frac{d-1}{\mathcal{H}^{d-1}\left(\partial B_{1}\right)}\left(\frac{1}{R^{d}}-\frac{1}{r^{d}}\right) & \text { for } & |x|<r \\
\frac{d-1}{\mathcal{H}^{d-1}\left(\partial B_{1}\right)} \frac{1}{R^{d}}+\frac{1}{\mathcal{H}^{d-1}\left(\partial B_{1}\right)} \frac{1}{|x|^{d}}\left(\mathrm{id}-d \frac{x}{|x|} \otimes \frac{x}{|x|}\right) & \text { for } & r<|x|<R \\
0 & \text { for } & |x|>R
\end{array}\right\} e_{1},
\end{aligned}
$$

where $e_{1}$ denotes the unit vector in the $x_{1}$-direction and id denotes the identity matrix.

Proof of Lemma 1.14. Because of $v^{R_{k}}=v_{k}$, cf. (1.33) and (4.27) this is a direct consequence of 4.31) and 4.32.

Proof of Lemma 4.2. We start by arguing that there is an explicit representation for $\omega_{r}^{R}$ given by

$$
\omega_{r}^{R}(x)=\left\{\begin{array}{lrr}
\frac{d-1}{\mathcal{H}^{d-1}\left(\partial B_{1}\right)}\left(\frac{1}{R^{d}}-\frac{1}{r^{d}}\right) x_{1} & \text { for } & |x|<r \\
\frac{d-1}{\mathcal{H}^{d-1}\left(\partial B_{1}\right)} \frac{x_{1}}{R^{d}}+\frac{1}{\mathcal{H}^{d-1}\left(\partial B_{1}\right)} \frac{x_{1}}{|x|^{d}} & \text { for } & r<|x|<R \\
0 & \text { for } & |x|>R
\end{array}\right\} .
$$

This is easily seen to hold: The normalization 4.30 is satisfied (because the function is odd), the so defined function is piecewise harmonic (because the dipole $\frac{x_{1}}{|x|^{d}}$ is the $\partial_{1}$-derivative of the fundamental solution), it satisfies the first jump condition at $|x|=r$ (because of $\left.\mathcal{H}^{d-1}\left(\partial B_{1}\right)=d\left|B_{1}\right|\right)$, and has no jump in the normal component at $|x|=r, R$ in view of formula 4.33 for $\nabla \omega_{r}^{R}$ (because of $\nu=\frac{x}{|x|}$ ).

We now give the argument for 4.31. By viii Green's formula and 4.30 there holds

$$
\begin{aligned}
& -\int_{B_{R}} \omega_{r}^{R} d \mu \stackrel{4.277, \sqrt{4.30}}{=}-\int_{B_{r}} \omega_{r}^{R} \Delta v^{R}-\int_{B_{R} \backslash \bar{B}_{r}} \omega_{r}^{R} \Delta v^{R} \\
& =-\int_{B_{r}} v^{R} \Delta \omega_{r}^{R}-\int_{B_{R} \backslash \bar{B}_{r}} v^{R} \Delta \omega_{r}^{R} \\
& +\int_{\partial B_{r}}\left[\omega_{r}^{R}\right] \nu \cdot \nabla v^{R}-\int_{\partial B_{r}} v^{R}\left[\nu \cdot \nabla \omega_{r}^{R}\right] \\
& -\int_{\partial B_{R}} \omega_{r}^{R} \nu \cdot \nabla v^{R}+\int_{\partial B_{R}} v^{R} \nu \cdot \nabla \omega_{r}^{R} \\
& \stackrel{4.27), 4.29}{=} \frac{1}{\left|B_{r}\right|} \int_{\partial B_{r}} x_{1} \nu \cdot \nabla v^{R} \text {. }
\end{aligned}
$$

We finally prove the telescoping formula 4.32 . To show this, it is convenient to split the functions into the affine part $x_{1}$ and the dipolar part $\frac{x_{1}}{|x|^{d}}$. The telescoping for

\footnotetext{
viii Note that under our assumption of $\mu\left(\partial B_{r}\right)=\mu\left(\partial B_{R}\right)=0$, the right-hand side is well-defined since $\mu$ does not charge the jump set of $\omega_{r}^{R}$ and the left-hand side is well-defined since the inner and outer normal distributional traces of $\nabla v^{R}$ on $\partial B_{r}$ agree since $\nabla v^{R} \in L^{p}\left(B_{R}\right)$ for $1 \leq p<\frac{d}{d-1}$ by Lemma 2.5. Since by [18, Prop. 3.2] both sides are well approximated by mollification (on $\mathbb{R}^{d}$ ), it is sufficient to show (4.31) for smooth approximation of $\mu$ and thus $v^{R}$.
} 
the dipolar part is obvious. The affine part can be written as

$$
\frac{(d-1) x_{1}}{\mathcal{H}^{d-1}\left(\partial B_{1}\right)}\left(\frac{1}{R^{d}} I(|x|<R)-\frac{1}{r^{d}} I(|x|<r)\right)
$$

and therefore is also telescoping.

\subsection{Linearization on nearby scales}

In this section we establish the main ingredient for the proof of Theorem 1.2 , namely Lemma 1.13, a linearization result on the level of (Eulerian) flux. The main difference with Theorem 1.2 is that it will only deal with nearby scales whereby Theorem 1.2 bridges scales. Before restating the result we recall a bit of notation.

For each $k \in[0, K]$, let $u_{k}$ be the (distributional) solution to

$$
\Delta u_{k}=\mu-1 \text { in } B_{R_{k}} \quad \text { and } \quad \nu \cdot \nabla u_{k}=\nu \cdot \bar{j}_{k} \text { on } \partial B_{R_{k}}
$$

and let $v_{k}$ be the solution to the Poisson problem with homogeneous flux boundary data

$$
\Delta v_{k}=\mu-1-c \text { in } B_{R_{k}} \quad \text { and } \quad \nu \cdot \nabla v_{k}=0 \text { on } \partial B_{R_{k}},
$$

so that (up to additive constants)

$$
u_{k}=v_{k}+\Phi_{k} .
$$

Let $\eta$ be a smooth and radial cutoff function as in (1.1), i.e.

$$
\eta \in C_{0}^{\infty}\left(B_{1}\right) \text { with } \int \eta=1, \sup \left|\nabla^{2} \eta\right| \lesssim 1 \quad \text { and we set } \eta_{R}:=\frac{1}{R^{d}} \eta\left(\frac{\cdot}{R}\right) .
$$

Lemma 1.13. For $R \in\left[R_{k+1}, R_{k}\right]$, there holds

$$
\begin{aligned}
\left|\int \eta_{R}\left(\bar{j}_{k}-\nabla u_{k}\right)\right| & \lesssim \frac{E_{k}}{R_{k}}, \\
\frac{1}{\left|B_{R_{k+1}}\right|}\left|\int_{B_{R_{k+1}}}\left(\bar{j}_{k}-\nabla u_{k}\right)\right| & \lesssim \frac{E_{k}}{R_{k}} .
\end{aligned}
$$

To appreciate this result, note that the vector field

$$
\sigma_{k}:=\bar{j}_{k}-\nabla u_{k}
$$

satisfies the homogeneous conditions

$$
\nabla \cdot \sigma_{k}=0 \text { in } B_{R_{k}} \quad \text { and } \quad \nu \cdot \sigma_{k}=0 \text { on } \partial B_{R_{k}},
$$

cf. (4.8) and 4.35). In Step 3 of the proof of Lemma 1.13 we will establish that $\bar{j}_{k} \approx$ id $-\nabla \psi_{k}$ on average, where $\psi_{k}$ is the Kantorovich potential. In particular, $\sigma_{k}$ is weakly close to a gradient

$$
\sigma_{k} \approx \nabla w_{k} \quad \text { on average, }
$$


namely the gradient of

$$
w_{k}(y):=\frac{1}{2}|y|^{2}-\psi_{k}(y)-u_{k}(y) .
$$

Smallness of large-scale averages of $\sigma_{k}$ will follow from the div-curl system 4.42 $\&$ (4.43) via a suitable representation in Steps 1 and 2 relating averages of $\sigma_{k}$ with averages of $\sigma_{k}-\nabla w_{k}$ (it is for this representation that it is crucial that $\sigma_{k}$ is divergence free and $\nabla w_{k}$ is a gradient field).

Proof of Lemma 1.13. For notational convenience, we can momentarily assume by scaling that $R_{k}=1$, drop the index $k$ and set $r:=R_{k+1}$ (and therefore consider radii $R \in[r, 1])$.

Let us first recall that thanks to Lemma 4.1, $r$ is chosen to be a good radius in the sense of $|\bar{j}|\left(\partial B_{r}\right)=0$. Since Lemma 2.5 implies $\nabla u \in L^{1}\left(B_{1}\right)$ we also have $|\sigma|\left(\partial B_{r}\right)=0$ (cf. (4.41)). We use $\nabla u \in L^{1}\left(B_{1}\right)$ once more together with the Lipschitz continuity of the Kantorovich potential $\psi$ to arrive at (cf. 4.44)

$$
\nabla w \in L^{1}\left(B_{1}\right) .
$$

STEP 1. We claim the representation formula of $\int \eta_{R} \sigma$ in terms of $\nabla w-\sigma$, restricting without loss of generality to the first component:

$$
\int \eta_{R} \sigma_{1}=\int_{B_{1}}\left(\eta_{R} e_{1}-\nabla \omega_{R}\right) \cdot(\sigma-\nabla w),
$$

where $\omega_{R}$ is a solution of (compare to 2.16)

$$
\Delta \omega_{R}=\partial_{1} \eta_{R} \text { in } B_{1} \quad \text { and } \quad \nu \cdot \nabla \omega_{R}=0 \text { on } \partial B_{1} .
$$

Indeed, it follows that (the smooth) $\eta_{R} e_{1}-\nabla \omega_{R}$ is divergence-free on $B_{1}$ with vanishing flux boundary data, so that the contribution of $\nabla w$ on the right-hand side of 4.46) vanishes. The contribution $\int_{B_{1}} \nabla \omega_{R} \cdot \sigma$ vanishes because of 4.42.

SteP 2. We claim the representation formula of $\frac{1}{\left|B_{r}\right|} \int_{B_{r}} \sigma$ in terms of $\nabla w-\sigma$ :

$$
\begin{aligned}
& \frac{1}{\left|B_{r}\right|} \int_{B_{r}} \sigma \\
& =\frac{d-1}{\mathcal{H}^{d-1}\left(\partial B_{1}\right)}\left(1-\frac{1}{r^{d}}\right) \int_{B_{r}}(\nabla w-\sigma) \\
& +\frac{1}{\mathcal{H}^{d-1}\left(\partial B_{1}\right)} \int_{B_{1} \backslash \bar{B}_{r}}\left((d-1) \mathrm{id}+\frac{1}{|x|^{d}}\left(\mathrm{id}-d \frac{x}{|x|} \otimes \frac{x}{|x|}\right)\right)(\nabla w-\sigma) .
\end{aligned}
$$

Indeed, in view of the formula 4.33 equation 4.48 assumes the form

$$
\frac{1}{\left|B_{r}\right|} \int_{B_{r}} \sigma_{1}=\int_{B_{1}} \nabla \omega_{r}^{1} \cdot(\nabla w-\sigma) .
$$

\footnotetext{
ix Note that this expression is well-defined since the measure $\sigma$ does not "charge" $\partial B_{r}$, the only place where $\nabla \omega_{r}^{1}$ is discontinuous, cf. beginning of this proof.
} 
By 4.42 and the divergence theorem (recall 1.38$)$ ) $\frac{1}{\left|B_{r}\right|} \int_{B_{r}} \sigma_{1}=\frac{1}{\left|B_{r}\right|} \int_{\partial B_{r}} x_{1} \nu \cdot \sigma$, so that by the jump condition in (4.29) we may write

$$
\frac{1}{\left|B_{r}\right|} \int_{B_{r}} \sigma_{1}=\int_{\partial B_{r}}\left[\omega_{r}^{1}\right] \nu \cdot \sigma .
$$

Recall that $\left[\omega_{r}^{1}\right]$ denotes the jump across $\partial B_{r}$ calculated as outside minus inside. By (4.42) and (4.29) (and since inner and outer distributional normal traces of $\sigma$ agree in view of 4.42 ) we then obtain ${ }^{\mathrm{x}}$

$$
\begin{aligned}
\frac{1}{\left|B_{r}\right|} \int_{B_{r}} \sigma_{1}= & -\int_{B_{r}} \sigma \cdot \nabla \omega_{r}^{1}-\int_{B_{1} \backslash \bar{B}_{r}} \sigma \cdot \nabla \omega_{r}^{1} \\
= & \int_{B_{r}}(\nabla w-\sigma) \cdot \nabla \omega_{r}^{1}+\int_{B_{1} \backslash \bar{B}_{r}}(\nabla w-\sigma) \cdot \nabla \omega_{r}^{1} \\
& \quad-\left(\int_{B_{r}} \nabla w \cdot \nabla \omega_{r}^{1}+\int_{B_{1} \backslash \bar{B}_{r}} \nabla w \cdot \nabla \omega_{r}^{1}\right) .
\end{aligned}
$$

The term in the parentheses vanishes since $\nabla \omega_{r}^{1}$ is weakly divergence-free in $B_{1}$ with vanishing normal component on $\partial B_{1}$, cf. (4.29). This proves (4.49).

SteP 3. We establish 4.43) in the following sense

$$
\left|\int_{B_{1}} \xi \cdot(\nabla w-\sigma)\right| \lesssim(\sup |\nabla \xi|+\sup |\xi|) \int_{\left(B_{6} \times \mathbb{R}^{d}\right) \cup\left(\mathbb{R}^{d} \times B_{6}\right)}|x-y|^{2} d \pi
$$

for any vector fields $\xi \in C_{0}^{\infty}\left(\mathbb{R}^{d}\right)^{d}$, and the same estimate with $B_{1}$ replaced by $B_{r}$. Indeed, we first note that by definition (4.41) and (4.44) of $\sigma$ and $w$ we have

$$
\nabla w-\sigma=\mathrm{id}-\nabla \psi-\bar{j} .
$$

Since $\pi$ i $\pi$ is supported on the graph $(\partial \psi(y), y)$ of the subgradient $\partial \psi$ of a convex function $\psi$, and since the $y$-marginal of $\pi$ is given by the Lebesgue measure $d y$, we have by the almost-everywhere differentiability of $\psi$

$$
\int \zeta d \pi=\int \zeta(\nabla \psi(y), y) d y
$$

for any test function $\zeta$. Hence we have by definition 2.21 of $\bar{j}=\int_{0}^{1} j_{t} d t$

$$
\begin{aligned}
& \int_{B_{1}} \xi(y) \cdot(y-\nabla \psi(y)) d y-\int_{B_{1}} \xi \cdot d \bar{j} \\
& \left.=\int_{0}^{1} \int\left(I\left(B_{1}\right) \xi\right)(y)-\left(I\left(B_{1}\right) \xi\right)((1-t) x+t y)\right) \cdot(y-x) d \pi d t .
\end{aligned}
$$

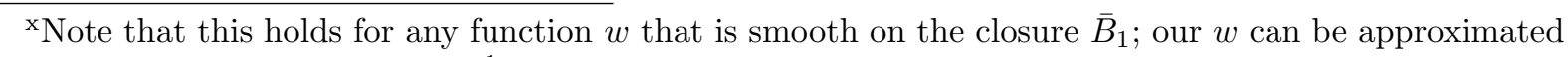
by such functions in the strong $L^{1}$-topology on the level of the gradients, cf. (4.45), which is sufficient to pass to the limit in the representation formula, since $\nabla \omega_{r}^{1}$ is in $L^{\infty}$.

${ }^{x i}$ Apart from the $L^{\infty}$ estimate this is the only place where we use that $\pi$ is an optimal transport plan. 
Hence the desired estimate splits into the obvious

$$
\begin{aligned}
\left|\int_{0}^{1} \int I(|y| \leq 1)(\xi(y)-\xi((1-t) x+t y)) \cdot(y-x) d \pi d t\right| & \\
\leq & \frac{1}{2} \sup |\nabla \xi| \int_{\mathbb{R}^{d} \times B_{1}}|x-y|^{2} d \pi
\end{aligned}
$$

and into

$$
\int_{0}^{1} \int|I(|y| \leq 1)-I(|(1-t) x+t y| \leq 1)||x-y| d \pi d t \lesssim \int_{\left(B_{6} \times \mathbb{R}^{d}\right) \cup\left(\mathbb{R}^{d} \times B_{6}\right)}|x-y|^{2} d \pi,
$$

for which we need that $R_{k}=1$ is a good radius in the sense of 4.20$)$, cf. Lemma 4.1 STEP 4. Proof of 4.39). In view of the representation 4.46), we need to apply 4.50 to $\xi=\eta_{R} e_{1}-\nabla \omega_{R}$. It follows from (4.47) that $\nabla \omega_{R}$ is of the same (scaling) form as $\eta_{R}$, cf. 4.38$)$, namely $\nabla \omega_{R}=\frac{1}{R^{d}} \nabla \omega\left(\frac{\dot{\bar{R}}}{)}\right)$ for some $\omega \in C^{\infty}\left(\bar{B}_{1}\right)$, which we may extend to an $\omega \in C_{0}^{\infty}\left(\mathbb{R}^{d}\right)$. In particular, we have

$$
\sup |\nabla \xi|+\sup |\xi| \lesssim \frac{1}{R^{d+1}}+\frac{1}{R^{d}} \lesssim 1,
$$

where we used closeness of scales in form of $R \geq r \gtrsim 1$, cf. 4.4. Hence 4.39) follows from 4.50).

STEP 5. Proof of 4.40). We recall (4.48) and apply first 4.50) with $\xi$ a constant unit vector $e$ and $R_{k}=1$ replaced by $r$. Since $r \gtrsim R$, cf. (4.4), we obtain

$$
\left|\left(1-\frac{1}{r^{d}}\right) \int_{B_{r}}(\nabla w-\sigma)\right| \lesssim \int_{\left(B_{6 r} \times \mathbb{R}^{d}\right) \cup\left(\mathbb{R}^{d} \times B_{6 r}\right)}|x-y|^{2} d \pi .
$$

We now turn to the second contribution to 4.48$)$. Let $\xi \in C^{\infty}\left(B_{1} \backslash \bar{B}_{r}\right)$. Appealing to 4.50 for both $R_{k}=1$ and $r \sim 1$, and to a Lipschitz extension of $\xi$ from $B_{1} \backslash \bar{B}_{r}$ to $B_{1}$ that preserves the $(\sup |\nabla \xi|+\sup |\xi|)$-norm, we obtain

$$
\left|\int_{B_{1} \backslash \bar{B}_{r}} \xi \cdot(\nabla w-\sigma)\right| \lesssim\left(\sup _{B_{1} \backslash \bar{B}_{r}}|\nabla \xi|+\sup _{B_{1} \backslash \bar{B}_{r}}|\xi|\right) \int_{\left(B_{6} \times \mathbb{R}^{d}\right) \cup\left(\mathbb{R}^{d} \times B_{6}\right)}|x-y|^{2} d \pi,
$$

which we apply to $\xi:=\frac{1}{\mathcal{H}^{d-1}\left(\partial B_{1}\right)}\left((d-1) \mathrm{id}+\frac{1}{|x|^{d}}\left(\mathrm{id}-d \frac{x}{|x|} \otimes \frac{x}{|x|}\right)\right) e$. Since, using again $r \gtrsim 1$, we have $\sup _{B_{1} \backslash \bar{B}_{r}}|\nabla \xi|+\sup _{B_{1} \backslash \bar{B}_{r}}|\xi| \lesssim 1$, and thus

$$
\begin{aligned}
&\left|\int_{B_{1} \backslash \bar{B}_{r}} \frac{1}{\mathcal{H}^{d-1}\left(\partial B_{1}\right)}\left((d-1) \mathrm{id}+\frac{1}{|x|^{d}}\left(\mathrm{id}-d \frac{x}{|x|} \otimes \frac{x}{|x|}\right)\right) \cdot(\nabla w-\sigma)\right| \\
& \lesssim \int_{\left(B_{6} \times \mathbb{R}^{d}\right) \cup\left(\mathbb{R}^{d} \times B_{6}\right)}|x-y|^{2} d \pi,
\end{aligned}
$$

and the desired estimate follows.

Let us recall that from Lemma 1.12 and Lemma 1.13 , we derived in the introduction Lemma 1.11, that is:

Lemma 1.11. For every $k \in[1, K-1]$,

$$
\left|\nabla \Phi_{k}(0)-\frac{1}{\left|B_{R_{k}}\right|} \int_{\partial B_{R_{k}}} x \nu \cdot \nabla v_{k-1}\right| \lesssim \frac{\beta\left(R_{k}\right)}{R_{k}} .
$$




\subsection{Representation of the cumulative shift}

In this section, we establish the last ingredient to the proof of Theorem 1.2, a crucial representation for the cumulative shift that links it to the flux of the Poisson equation, i.e. Proposition 1.10, which we now restate. Recall the definition 4.6) of $\Phi_{k}$ and (4.35) of $u_{k}$.

Proposition 1.10. For every $k \in[0, K-1]$ and $R \in\left[R_{k+1}, R_{k}\right]$, we have the two estimates on the cumulative flux

$$
\left|\sum_{\ell=0}^{k} \nabla \Phi_{\ell}(0)-\frac{1}{\left|B_{R_{k}}\right|} \int_{\partial B_{R_{k}}} x \nu \cdot \nabla u_{0}\right| \lesssim \frac{\beta\left(R_{k}\right)}{R_{k}}
$$

and

$$
\left|\sum_{\ell=0}^{k-1} \nabla \Phi_{\ell}(0)+\int \eta_{R} \nabla u_{k}-\int \eta_{R} \nabla u_{0}\right| \lesssim \frac{\beta\left(R_{k}\right)}{R_{k}} .
$$

Proof. We start with the proof of (4.52) and first point out that from (4.28) (and the fact that 4.27) and 4.36) are the same boundary problem and thus $v_{k}=v^{R_{k}}$ ),

$$
\frac{1}{\left|B_{R_{k}}\right|} \int_{\partial B_{R_{k}}} x \nu \cdot \nabla v_{0}=\sum_{\ell=1}^{k} \frac{1}{\left|B_{R_{\ell}}\right|} \int_{\partial B_{R_{\ell}}} x \nu \cdot \nabla v_{\ell-1} .
$$

Therefore, using that $u_{0}=v_{0}+\Phi_{0}$ (recall 4.37) ) and the divergence theorem (cf. (1.38)) we have

$$
\nabla \Phi_{0}(0)+\sum_{\ell=1}^{k} \frac{1}{\left|B_{R_{\ell}}\right|} \int_{\partial B_{R_{\ell}}} x \nu \cdot \nabla v_{\ell-1}=\frac{1}{\left|B_{R_{k}}\right|} \int_{\partial B_{R_{k}}} x \nu \cdot \nabla u_{0} .
$$

We conclude from Lemma 1.11 that

$$
\begin{aligned}
\left|\sum_{\ell=0}^{k} \nabla \Phi_{\ell}(0)-\frac{1}{\left|B_{R_{k}}\right|} \int_{\partial B_{R_{k}}} x \nu \cdot \nabla u_{0}\right| & =\left|\sum_{\ell=1}^{k}\left(\nabla \Phi_{\ell}(0)-\frac{1}{\left|B_{R_{\ell}}\right|} \int_{\partial B_{R_{\ell}}} x \nu \cdot \nabla v_{\ell-1}\right)\right| \\
& \stackrel{4.51}{\lesssim} \sum_{\ell=1}^{k} \frac{\beta\left(R_{\ell}\right)}{R_{\ell}} \stackrel{\text { 4.1. }}{\lesssim} \frac{\beta\left(R_{k}\right)}{R_{k}},
\end{aligned}
$$

which is 4.52.

From 4.52 , estimate 4.53 follows via the identity

$$
\nabla \Phi_{k}(0)-\frac{1}{\left|B_{R_{k}}\right|} \int_{\partial B_{R_{k}}} x \nu \cdot \nabla u_{0}=\int \eta_{R} \nabla u_{k}-\int \eta_{R} \nabla u_{0}
$$


for $R \in\left[R_{k+1}, R_{k}\right]$. Notice that since $\nabla u_{k}=\nabla v_{k}+\nabla \Phi_{k}$ (recall (4.37) ) and by the mean-value property for the harmonic $\nabla \Phi_{k}$ in form of $\int \eta_{R} \nabla \Phi_{k}=\nabla \Phi_{k}(0)$, cf. the normalization in 4.38, this identity reduces to

$$
-\frac{1}{\left|B_{R_{k}}\right|} \int_{\partial B_{R_{k}}} x \nu \cdot \nabla u_{0}=\int \eta_{R} \nabla v_{k}-\int \eta_{R} \nabla u_{0} .
$$

By the boundary condition $\nu \cdot \nabla v_{k}=0$ on $\partial B_{R_{k}}$ (cf. 4.36) ) the last statement takes the form

$$
\frac{1}{\left|B_{R_{k}}\right|} \int_{\partial B_{R_{k}}} x \nu \cdot \nabla\left(v_{k}-u_{0}\right)=\int \eta_{R} \nabla\left(v_{k}-u_{0}\right) .
$$

Since $\nabla\left(v_{k}-u_{0}\right)$ is harmonic in $B_{R_{k}}$, cf. 4.36) \& 4.35), the latter holds by the divergence theorem (see (1.38) ) and the mean-value property in form of

$$
\int \eta_{R} \nabla\left(v_{k}-u_{0}\right)=\frac{1}{\left|B_{R_{k}}\right|} \int_{B_{R_{k}}} \nabla\left(v_{k}-u_{0}\right) .
$$

Let us close this section by estimating the distance between spherical and radial averages of $\nabla u$. This implies that when identifying the cumulated shift, one can use either of them.

Lemma 4.3. For every $k \in[0, K-1]$ and $R \in\left[R_{k+1}, R_{k}\right]$,

$$
\left|\int \eta_{R} \nabla u_{0}-\frac{1}{\left|B_{R_{k}}\right|} \int_{\partial B_{R_{k}}} x \nu \cdot \nabla u_{0}\right|^{2} \lesssim \beta(R) .
$$

Proof. By 4.10), it is enough to prove that

$$
\left|\int \eta_{R} \partial_{1} u_{0}-\frac{1}{\left|B_{R_{k}}\right|} \int_{\partial B_{R_{k}}} x_{1} \nu \cdot \nabla u_{0}\right|^{2} \lesssim \frac{1}{\left|B_{R_{k}}\right|} W_{B_{R_{k}}}^{2}(\mu, \kappa) .
$$

By scaling we may assume that $R_{k}=1$. The argument is somewhat reminiscent of the one leading to Lemma 4.2 . We claim that there exists $\omega \in C^{\infty}\left(\bar{B}_{1}\right)$ with $\sup _{B_{1}}|\nabla \omega| \lesssim 1$ such that for $\zeta \in C_{c}^{\infty}\left(\mathbb{R}^{d}\right)$,

$$
\int \eta_{R} \partial_{1} \zeta-\frac{1}{\left|B_{1}\right|} \int_{\partial B_{1}} x_{1} \nu \cdot \nabla \zeta=\int_{B_{1}} \omega \Delta \zeta
$$

Then, testing this equation with $\zeta=u_{0}$ (thanks to a simple approximation argument) and observing that $\int_{B_{1}} \omega=0$ (choosing $\zeta=|x|^{2}$ ), we would obtain 4.55) from

$$
\left|\int_{B_{1}} \omega d(\mu-\kappa)\right|^{2} \lesssim W_{B_{1}}^{2}(\mu, \kappa) .
$$

Let $\hat{\omega}$ be a solution of

$$
\Delta \hat{\omega}=-\eta_{R}-\frac{d-1}{\left|B_{1}\right|} \text { in } B_{1} \quad \text { and } \quad \nu \cdot \nabla \hat{\omega}=-\frac{1}{\left|B_{1}\right|} \text { on } \partial B_{1},
$$


which is solvable since $\int \eta_{R}=1$ and $\mathcal{H}^{d-1}\left(\partial B_{1}\right)=d\left|B_{1}\right|$. Since $\eta_{R}$ is radially symmetric so is $\hat{\omega}$ and it is readily checked that $\omega:=\partial_{1} \hat{\omega}=\frac{d \hat{\omega}}{d r}(r) \frac{x_{1}}{r}$ solves

$$
\Delta \omega=-\partial_{1} \eta_{R} \text { in } B_{1} \quad \text { and } \quad \nu \cdot \nabla \omega=0, \omega=-\frac{x_{1}}{\left|B_{1}\right|} \text { on } \partial B_{1}
$$

which is equivalent to 4.56 . Finally since $R \sim 1$ we have $\sup _{B_{1}}|\nabla \omega| \lesssim 1$.

\subsection{Proof of Theorem 1.2}

We start this section with a technical lemma that we will need for the proof of Theorem 1.2.

Lemma 4.4. For every $k \in[0, K-1]$ and every $R \in\left[R_{k+1}, R_{k}\right]$, there holds

$$
\begin{aligned}
\left|\int \eta_{R} \bar{j}_{k}-\int \eta_{R}(x)(y-x) d \pi_{k}\right| & \lesssim \frac{E_{k}}{R_{k}} \\
\left|\int \eta_{R}(x) d \pi-1\right| & \lesssim \frac{\sqrt{E_{k}}}{R_{k}} \ll 1, \\
\left|\int \eta_{R} \nabla u_{k}\right| & \lesssim \sqrt{E_{k}} .
\end{aligned}
$$

Proof. We start with (4.57) and note that by definition (4.7) of $\bar{j}$, the left-hand side can be written as

$$
\begin{aligned}
\int_{0}^{1} \int\left(\eta_{R}((1-t) x+t y)-\eta_{R}(x)\right) & (y-x) d \pi_{k} d t \\
= & \int_{0}^{1} \int_{0}^{t} \int \nabla \eta_{R}((1-s) x+s y) \cdot(y-x)^{2} d \pi_{k} d s d t
\end{aligned}
$$

and thus

$$
\begin{aligned}
& \left|\int \eta_{R} \bar{j}_{k}-\int \eta_{R}(x)(y-x) d \pi_{k}\right| \\
& \leq \frac{1}{2} \sup \left|\nabla \eta_{R}\right| \int_{0}^{1} \int I(|(1-t) x+t y|<R)|y-x|^{2} d \pi_{k} d t \\
& \underset{\underbrace{2.34}_{(4.38}}{\lesssim} \frac{1}{R^{d+1}} \int_{\left(B_{3 R} \times \mathbb{R}^{d}\right) \cup\left(\mathbb{R}^{d} \times B_{3 R}\right)}|x-y|^{2} d \pi_{k} .
\end{aligned}
$$

Combining this with $R \sim R_{k}$ (cf. (4.11), we obtain (4.57).

We turn to 4.58 and reformulate the left-hand side: Clearly, since the $x$-component of the transference plan is not affected by the shift (4.5), we may replace $\pi$ by $\pi_{k}$; the $y$-marginal of $\pi_{k}$ is the Lebesgue measure and by the normalization in 4.38) we have $1=\int \eta_{R}(y) d \pi_{k}$, so that as above

$$
\begin{aligned}
\left|\int \eta_{R}(x) d \pi-1\right| & \leq \frac{1}{2} \sup \left|\nabla \eta_{R}\right| \int_{0}^{1} \int I(|(1-t) x+t y|<R)|y-x| d \pi_{k} d t \\
& \lesssim \frac{1}{R^{d+1}} \int_{\left(B_{3 R} \times \mathbb{R}^{d}\right) \cup\left(\mathbb{R}^{d} \times B_{3 R}\right)}|x-y| d \pi_{k} .
\end{aligned}
$$


In view of $R \sim R_{k}$ it is sufficient to show the estimate

$$
\frac{1}{R_{k}^{d}} \int_{\left(B_{3 R_{k}} \times \mathbb{R}^{d}\right) \cup\left(\mathbb{R}^{d} \times B_{3 R_{k}}\right)}|x-y| d \pi_{k} \lesssim \sqrt{E_{k}} .
$$

which follows from the Cauchy-Schwarz inequality and 2.36).

We finally turn to 4.59 ) and start by applying (4.39), yielding

$$
\left|\int \eta_{R}\left(\bar{j}_{k}-\nabla u_{k}\right)\right| \lesssim \frac{E_{k}}{R_{k}}
$$

Noting that $\frac{E_{k}}{R_{k}} \ll \sqrt{E_{k}}$ thanks to 4.11) and 4.3), it is enough to establish 4.59) with $\nabla u_{k}$ replaced by $\bar{j}_{k}$. We observe that by definition (4.7) we have

$$
\begin{aligned}
\left|\int \eta_{R} \bar{j}_{k}\right| & =\left|\int_{0}^{1} \int \eta_{R}((1-t) x+t y)(y-x) d \pi_{k} d t\right| \\
& \stackrel{4.38}{\lesssim} \int I(|(1-t) x+t y|<R)|x-y| d \pi_{k},
\end{aligned}
$$

so that the argument continuous as for 4.58 .

Now we may prove Theorem 1.2 which we restate and reformulate here for the reader's convenience.

Theorem 1.2. Let

$$
h_{R}:=\int \eta_{R} \nabla u_{0}
$$

Then, for every $R \in\left[R_{K}, R_{0}\right]$,

$$
\left|\int \eta_{R}(x)\left(y-x-h_{R}\right) d \pi\right|+\left|\int \eta_{R}\left(y-h_{R}\right)\left(y-x-h_{R}\right) d \pi\right| \lesssim \frac{\beta(R)}{R}
$$

and

$$
\begin{aligned}
\sup \left\{\left|y-x-h_{R}\right|:(x, y) \in \operatorname{Spt} \pi \cap\left[( B _ { R } \times \mathbb { R } ^ { d } ) \cup \left(\mathbb{R}^{d}\right.\right.\right. & \left.\left.\left.\times B_{R}\left(h_{R}\right)\right)\right]\right\} \\
& \lesssim R\left(\frac{\beta(R)}{R^{2}}\right)^{\frac{1}{d+2}} .
\end{aligned}
$$

Proof. Step 1. We actually start with the proof of (4.63). We claim that

$$
\frac{1}{\left|B_{2 R}\right|} \int_{\left(B_{2 R} \times \mathbb{R}^{d}\right) \cup\left(\mathbb{R}^{d} \times B_{2 R}\left(h_{R}\right)\right)}\left|x-y+h_{R}\right|^{2} d \pi \lesssim \beta(R) .
$$

Combining this with the $L^{\infty}$ bound $(2.33)$ applied to $\widetilde{\pi}=\left(\mathrm{id}, \mathrm{id}-h_{R}\right) \# \pi$ would yield 4.63. 
Let $k \in[0, K-1]$ be such that $R \in\left[R_{k+1}, R_{k}\right]$. Undoing the iterative shift (4.5), estimate (4.11) takes the form

$$
\frac{1}{\left|B_{6 R_{k}}\right|} \int_{\left(B_{6 R_{k}} \times \mathbb{R}^{d}\right) \cup\left(\mathbb{R}^{d} \times B_{6 R_{k}}\left(\widetilde{h}_{k}\right)\right)}\left|x-y+\widetilde{h}_{k}\right|^{2} d \pi \lesssim \beta\left(R_{k}\right)
$$

with shift $\widetilde{h}_{k}:=\sum_{\ell=0}^{k} \nabla \Phi_{\ell}(0)$. By (4.52), 4.54) (and 4.1)), we have

$$
\left|\widetilde{h}_{k}-h_{R}\right| \lesssim \frac{\beta\left(R_{k}\right)}{R_{k}}+\beta^{\frac{1}{2}}(R) \lesssim \beta^{\frac{1}{2}}(R)
$$

Because $\beta(R) \ll R^{2}$ (cf. (4.3)) this in particular implies that $B_{2 R}\left(h_{R}\right) \subset B_{6 R_{k}}\left(\widetilde{h}_{k}\right)$. We may thus estimate

$$
\begin{aligned}
& \frac{1}{\left|B_{2 R}\right|} \int_{\left(B_{2 R} \times \mathbb{R}^{d}\right) \cup\left(\mathbb{R}^{d} \times B_{2 R}\left(h_{R}\right)\right)}\left|x-y+h_{R}\right|^{2} d \pi \\
& \lesssim \frac{1}{\left|B_{2 R}\right|} \int_{\left(B_{2 R} \times \mathbb{R}^{d}\right) \cup\left(\mathbb{R}^{d} \times B_{2 R}\left(h_{R}\right)\right)}\left(\left|x-y+\widetilde{h}_{k}\right|^{2}+\left|h_{R}-\widetilde{h}_{k}\right|^{2}\right) d \pi
\end{aligned}
$$

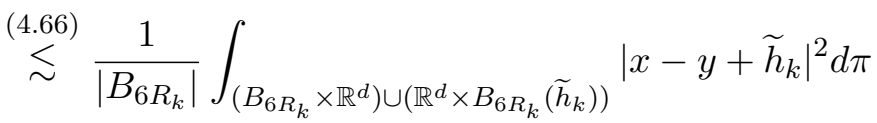

$$
\begin{aligned}
& +\left(\frac{1}{\left|B_{2 R}\right|} \int_{\left(B_{2 R} \times \mathbb{R}^{d}\right) \cup\left(\mathbb{R}^{d} \times B_{2 R}\left(h_{R}\right)\right)} d \pi\right) \beta(R) \\
& \stackrel{4.65}{\lesssim} \beta(R)
\end{aligned}
$$

This proves 4.64).

SteP 2. We now turn to the proof of 4.62$)$. Let again $k \in[0, K-1]$ be such that $R \in\left[R_{k+1}, R_{k}\right]$. Writing

$$
\int \eta_{R}(x)(y-x) d \pi_{k}-\int \eta_{R} \nabla u_{k}=\int \eta_{R}(x)(y-x) d \pi_{k}-\int \eta_{R} \bar{j}_{k}+\int \eta_{R}\left(\nabla u_{k}-\bar{j}_{k}\right)
$$

and evoking (4.57) of Lemma 4.4 and 4.39), we obtain

$$
\left|\int \eta_{R}(x)(y-x) d \pi_{k}-\int \eta_{R} \nabla u_{k}\right| \lesssim \frac{E_{k}}{R_{k}} .
$$

Appealing to the iterative definition (4.5) of $\pi_{k}$ this is equivalent to

$$
\left|\int \eta_{R}(x)\left(y-x-\sum_{\ell=0}^{k-1} \nabla \Phi_{\ell}(0)\right) d \pi-\int \eta_{R} \nabla u_{k}\right| \lesssim \frac{E_{k}}{R_{k}} .
$$

We now use 4.58) \& 4.59) of Lemma 4.4 in the form of

$$
\left|\int \eta_{R} \nabla u_{k}-\int \eta_{R}(x)\left(\int \eta_{R} \nabla u_{k}\right) d \pi\right| \lesssim \frac{E_{k}}{R_{k}}
$$


to reformulate this as

$$
\left|\int \eta_{R}(x)(y-x) d \pi-\left(\int \eta_{R}(x) d \pi\right)\left(\sum_{\ell=0}^{k-1} \nabla \Phi_{\ell}(0)+\int \eta_{R} \nabla u_{k}\right)\right| \lesssim \frac{E_{k}}{R_{k}} .
$$

By 4.53, 4.11) and the monotonicity of $\frac{\beta(R)}{R}$ we get the first part of 4.62 ,

$$
\left|\int \eta_{R}(x)\left(y-x-h_{R}\right) d \pi\right| \lesssim \frac{\beta(R)}{R}
$$

By (4.38),

$$
\begin{aligned}
\mid \int\left(\eta_{R}(x)-\eta_{R}\left(y-h_{R}\right)\right)(y-x- & \left.h_{R}\right) d \pi \mid \\
& \lesssim \frac{1}{R^{d+1}} \int_{\left(B_{R} \times \mathbb{R}^{d}\right) \cup\left(\mathbb{R}^{d} \times B_{R}\left(h_{R}\right)\right)}\left|x-y+h_{R}\right|^{2} d \pi,
\end{aligned}
$$

and thus the second part of 4.62 follows from 4.64 ).

We finally derive Corollary 1.1, which we recall for the reader's convenience, from Theorem 1.2,

Corollary 1.1. Let $\mu$ be such that for some $0 \leq \alpha<1$, and some $\bar{R} \gg 1$, there holds

$$
\frac{1}{\left|B_{R}\right|} W_{B_{R}}^{2}(\mu, \kappa) \leq C R^{\alpha} \quad \forall R \in[1, \bar{R}] .
$$

Then, assuming for simplicity that $\mu\left(B_{\bar{R}}\right)=\left|B_{\bar{R}}\right|$, if $\pi$ is the optimal coupling for $W_{B_{\bar{R}}}(\mu, 1)$, for every $\bar{R} \geq R \gtrsim 1$,

$$
\left|\int \eta_{R}(x)\left(y-x-h_{R}\right) d \pi\right| \lesssim \frac{1}{R^{1-\alpha}}
$$

where for $R>0, h_{R}=\int \eta_{R} \nabla u$ with $u$ the up to an additive constant unique (distributional) solution of the Poisson equation

$$
\Delta u=\mu-1 \quad \text { in } B_{\bar{R}} \quad \text { and } \quad \nu \cdot \nabla u=0 \quad \text { on } \partial B_{\bar{R}} .
$$

Proof. The statement follows directly from Theorem 1.2 provided we may choose $R_{0}=\bar{R}$. To prove this, notice that on the one hand Spt $\pi \subset B_{\bar{R}} \times B_{\bar{R}}$ implies

$$
\frac{1}{\left|B_{6 \bar{R}}\right|} \int_{\left(B_{6 \bar{R}} \times \mathbb{R}^{d}\right) \cup\left(\mathbb{R}^{d} \times B_{6 \bar{R}}\right)}|x-y|^{2} d \pi \leq \frac{1}{\left|B_{\bar{R}}\right|} \int_{\left(B_{\bar{R}} \times \mathbb{R}^{d}\right) \cup\left(\mathbb{R}^{d} \times B_{\bar{R}}\right)}|x-y|^{2} d \pi,
$$

and thus $R_{0}=\bar{R}$ and $\Phi_{0}=0$ satisfy the conclusions (4.11) and 4.12 of the Campanato iteration. On the other hand, $R_{0}$ is a good radius in the sense of Lemma 4.1 (since having no transport across $\partial B_{R_{0}}$, the left-hand sides of 4.19) and 4.20) are both equal to zero). Therefore $R_{0}=\bar{R}$ is indeed admissible. 


\section{References}

[1] M. Ajtai, J. Komlós, and G. Tusnády, On optimal matchings., Combinatorica 4 (1984), 259-264.

[2] L. Ambrosio and A. Figalli, On the regularity of the pressure field of Brenier's weak solutions to incompressible Euler equations, Calc. Var. Partial Differential Equations 31 (2008), no. 4, 497-509.

[3] L. Ambrosio, N. Fusco, and D. Pallara, Functions of bounded variation and free discontinuity problems, Oxford Mathematical Monographs, The Clarendon Press, Oxford University Press, New York, 2000.

[4] L. Ambrosio, N. Gigli, and G. Savaré, Gradient flows in metric spaces and in the space of probability measures, second ed., Lectures in Mathematics ETH Zürich, Birkhäuser Verlag, Basel, 2008.

[5] L. Ambrosio, F. Glaudo, and D. Trevisan, On the optimal map in the 2dimensional random matching problem, Discrete Contin. Dyn. Syst. 39 (2019), no. $12,7291-7308$.

[6] L. Ambrosio, F. Stra, and D. Trevisan, A PDE approach to a 2-dimensional matching problem, Probab. Theory Relat. Fields 173 (2019), no. 1-2, 433-477.

[7] S. Armstrong, S. J. Ferguson, and T. Kuusi, Homogenization, linearization, and large-scale regularity for nonlinear elliptic equations, Communications on Pure and Applied Mathematics (2020).

[8] S. Armstrong, T. Kuusi, and J.-C. Mourrat, Quantitative stochastic homogenization and large-scale regularity, Grundlehren der Mathematischen Wissenschaften, vol. 352, Springer, Cham, 2019.

[9] S. Armstrong and C. K. Smart, Quantitative stochastic homogenization of convex integral functionals, Ann. Sci. Éc. Norm. Supér. (4) 49 (2016), no. 2, 423-481.

[10] M. Avellaneda and F.-H. Lin, Compactness methods in the theory of homogenization, Comm. Pure Appl. Math. 40 (1987), no. 6, 803-847.

[11] F. Barthe and C. Bordenave, Combinatorial optimization over two random point sets., Séminaire de probabilités XLV, Cham: Springer, 2013, pp. 483-535.

[12] R. J. Berman, Convergence rates for discretized monge-ampère equations and quantitative stability of optimal transport, arXiv:1803.00785 (2018).

[13] Y. Brenier, Minimal geodesics on groups of volume-preserving maps and generalized solutions of the Euler equations, Comm. Pure Appl. Math. 52 (1999), no. $4,411-452$.

[14] Y. Brenier and G. Loeper, A geometric approximation to the Euler equations: the Vlasov-Monge-Ampère system, Geom. Funct. Anal. 14 (2004), no. 6, 1182 1218.

[15] L. A. Caffarelli, Some regularity properties of solutions of Monge Ampère equation, Comm. Pure Appl. Math. 44 (1991), no. 8-9, 965-969. 
[16] _ The regularity of mappings with a convex potential, J. Amer. Math. Soc. 5 (1992), no. 1, 99-104.

[17] S. Caracciolo, C. Lucibello, G. Parisi, and G. Sicuro, Scaling hypothesis for the euclidean bipartite matching problem, Physical Review E 90 (2014), no. 1.

[18] G.-Q. Chen and H. Frid, On the theory of divergence-measure fields and its applications., Bol. Soc. Bras. Mat., Nova Sér. 32 (2001), no. 3, 401-433.

[19] G.-Q. Chen and H. Frid, Extended divergence-measure fields and the Euler equations for gas dynamics, Comm. Math. Phys. 236 (2003), no. 2, 251-280.

[20] G. De Philippis and A. Figalli, Partial regularity for optimal transport maps, Publ. Math. Inst. Hautes Études Sci. 121 (2015), 81-112.

[21] L. Dupaigne, Stable solutions of elliptic partial differential equations, Chapman \& Hall/CRC Monographs and Surveys in Pure and Applied Mathematics, vol. 143, Chapman \& Hall/CRC, Boca Raton, FL, 2011.

[22] A. Figalli and Y.-H. Kim, Partial regularity of Brenier solutions of the MongeAmpère equation, Discrete Contin. Dyn. Syst. 28 (2010), no. 2, 559-565.

[23] J. Fischer and S. Neukamm, Optimal homogenization rates in stochastic homogenization of nonlinear uniformly elliptic equations and systems, arXiv:1908.02273 (2019).

[24] A. Gloria, S. Neukamm, and F. Otto, A regularity theory for random elliptic operators, ArXiv e-prints (2014).

[25] M. Goldman, M. Huesmann, and F. Otto, A large-scale regularity theory for the Monge-Ampere equation with rough data and application to the optimal matching problem, arXiv:1808.09250 (2018).

[26] M. Goldman and F. Otto, A variational proof of partial regularity for optimal transportation maps, arXiv:1704.05339 (2017).

[27] M. Huesmann and K.-T. Sturm, Optimal transport from Lebesgue to Poisson, Ann. Probab. 41 (2013), no. 4, 2426-2478.

[28] M. Ledoux, On optimal matching of Gaussian samples, Zap. Nauchn. Sem. S.Peterburg. Otdel. Mat. Inst. Steklov. (POMI) 457 (2017), no. Veroyatnost' i Statistika. 25, 226-264.

[29] _ On optimal matching of Gaussian samples II, preprint (2018).

[30] Q. Mérigot, A. Delalande, and F. Chazal, Quantitative stability of optimal transport maps and linearization of the 2-wasserstein space, arXiv:1910.05954 (2019).

[31] F. Santambrogio, Optimal transport for applied mathematicians, Progress in Nonlinear Differential Equations and their Applications, vol. 87, Birkhäuser/Springer, Cham, 2015, Calculus of variations, PDEs, and modeling.

[32] _ Regularity via duality in calculus of variations and degenerate elliptic PDEs, J. Math. Anal. Appl. 457 (2018), no. 2, 1649-1674. 
[33] M. Talagrand, Upper and lower bounds for stochastic processes: modern methods and classical problems, vol. 60, Springer Science \& Business Media, 2014.

[34] G. M. Troianiello, Elliptic differential equations and obstacle problems, The University Series in Mathematics, Plenum Press, New York, 1987.

[35] C. Villani, Topics in optimal transportation, Graduate Studies in Mathematics, vol. 58, American Mathematical Society, Providence, RI, 2003.

[36] _ Optimal transport: old and new, Grundlehren der Mathematischen Wissenschaften, vol. 338, Springer-Verlag, Berlin, 2009. 